UTTG-13-06

hep-th/yymmnnn

\title{
Quantum Deconstruction of 5D SQCD
}

\author{
Edoardo Di Napoli ${ }^{(1)}$ and Vadim S. Kaplunovsky ${ }^{(2)}$ \\ (1) University of North Carolina \\ (2) University of Texas at Austin, \\ Dept. of Physics and Astronomy \\ Theory Group, Physics Department, \\ CB \#3255, Phillips Hall \\ 1 University Station, C1608 \\ Chapel Hill, NC 27599-3255, USA \\ Austin, TX 78712-1608, USA \\ edodin@physics.unc.edu \\ vadim@physics.utexas.edu
}

\begin{abstract}
We deconstruct the fifth dimension of 5D SCQD with general numbers of colors and flavors and general 5D Chern-Simons level; the latter is adjusted by adding extra quarks to the $4 \mathrm{D}$ quiver. We use deconstruction as a non-stringy UV completion of the quantum 5D theory; to prove its usefulness, we compute quantum corrections to the $\mathrm{SQCD}_{5}$ 's prepotential. We also explore the moduli/parameter space of the deconstructed $\mathrm{SQCD}_{5}$ and show that for $\left|k_{\mathrm{cs}}\right|<n_{c}-\frac{1}{2} n_{f}$ it continues to negative values of $1 / g_{5 d}^{2}$. In many cases there are flop transitions connecting $\mathrm{SQCD}_{5}$ to exotic $5 \mathrm{D}$ theories such as $E_{0}$, and we present several examples of such transitions. We compare deconstruction to brane-web engineering of the same $\mathrm{SQCD}_{5}$ and show that the phase diagram is the same in both cases; indeed, the two UV completions are in the same universality class, although they are not dual to each other. Hence, the phase structure of an $\mathrm{SQCD}_{5}$ (and presumably any other 5D gauge theory) is inherently five-dimensional and does not depends on a UV completion.
\end{abstract}

* Article based on research supported by the US National Science Foundation (grant PHY-0455649) and by the US Department of Energy (grant DE-FG02-06ER41418) 


\section{Contents}

1 Introduction $\quad 3$

2 Semiclassical (De) Construction 12

3 On Chern-Simons Couplings and Extra 4D Flavors 24

4 Quantum Deconstruction of the Gauge Couplings 31

5 Quantum Baryonic Branches $\quad 42$

$6 \quad S U(2)$ Examples of Phase Structures and Flop Transitions. 49

6.1 The $\widetilde{D}_{0}$ Model: $n_{c}=2, n_{f}=0, \Delta F=1 \ldots \ldots \ldots 1$

6.2 The $D_{0}$ Model: $n_{c}=2, n_{f}=0, \Delta F=2 \ldots \ldots \ldots$. . . . . . . 62

6.3 Models with Flavor: $n_{c}=n_{f}=2 \ldots \ldots \ldots$. . . . . . . . 70

7 Deconstruction / String Universality $\quad 91$ 


\section{Introduction}

\section{Motivation:}

Five-dimensional $\mathcal{N}=1$ SUSY gauge theories appear to be well understood. Indeed, constraints due to 8 supersymmetries combined with gauge invariance are so powerful that many low-energy properties of the theory — such as geometry of its moduli space — can be cal-

culated exactly $[1,2]$. But all such calculations presume that SUSY and gauge invariance persist on the quantum level of the 5D theory. In other words, all our knowledge assumes some kind of a UV completion which keeps these symmetries manifest.

In 5D all interactive field theories are non-renormalizable, so $4 \mathrm{D}$-style perturbative UV cutoffs such as DR or covariant higher-derivative terms are of no use. Instead, most research into 5D gauge theories embeds them into string or $\mathrm{M}$ theory as a UV completion. For example, one may (1) compactify M theory on a singular Calabi-Yau threefold [3, 4, 5, 6, 7], or (2) make a web of $(p, q)$ five-branes in type IIB string theory [8, 9, 10, 11, 12], or (3) put D4-brane probes of type IIA string in a background of D8 branes and O8 orientifold planes $[1,13,2]$. In such completions, one may use the full power of string/M theory to derive the global geometry of the 5D moduli space, including 'flop' transition to different 5D phases, sometimes involving strongly-coupled sectors with non-trivial IR fixed points [14]. But unfortunately, in string context it is hard to tell whether all these phases are made of the same QFT-level degrees of freedom, or perhaps they follow from different sectors of the string theory. In other words, we don't know if the whole phase diagram is an inherent property of the 5D gauge theory (regardless of a UV regulator), or perhaps some phases are artefacts of embedding into string theory.

To resolve this issue, we need to compare phase structures of different UV completions of the same 5D theory. Since all 'stringy' completions are dual to each other as string/M theories, there is no use in comparing them to each other. Thus we need a non-stringy regulator such as lattice. But the Euclidean 5D lattice breaks SUSY; also, it's hard to latticize the Chern-Simons interactions of the gauge fields. Instead, we shall use a lattice / continuum hybrid known as dimensional deconstruction [15, 16, 17, 18, 19]: 4 dimensions out of five remain continuous while the fifth dimension becomes discrete. 
In an earlier article [20], we have used deconstruction as a UV completion of 5D SYM theories with maximal Chern-Simons levels $k_{\mathrm{cs}}=n_{c}$. In this article we extend this method to $\mathrm{SQCD}_{5}$ with general numbers of colors and flavors and all allowed Chern-Simons levels. Our main results are as follows: (1) We develop the quantum aspects of deconstruction technology. In particular, we show how to convert the exact non-perturbative data (which obtain at the $4 \mathrm{D}$ level of deconstructed theories) into the 5D moduli dependence of gauge couplings, and hence into 5D phase structures. (2) We prove universality of $\mathrm{SQCD}_{5}$ phase diagrams: for any choice of $n_{c}, n_{f}$, and $k_{\mathrm{cs}}$, the deconstructed $\mathrm{SQCD}_{5}$ and the string embedding of the same 5D theory are in the same universality class and have identical phase diagrams. This strongly suggests that other UV regulators are also in the same universality class and hence the phase diagrams are inherent properties of the 5D theories.

\section{Overview of 5D SQCD:}

Now that we have made out intentions clear, let us briefly introduce two subjects that may be unfamiliar to some readers, namely the SQCD in 5D, and the dimensional deconstruction. We start with the the basic features of 5D gauge theories with $\mathcal{N}=1$ SUSY (which in five dimensions means 8 rather than 4 supercharges) [2]. First of all, there are two kinds of supermultiplets, vector and hyper: a vector multiplet contains a gauge field $A_{\mu}$, a Dirac fermion (4 complex components), and a real scalar; a hyper multiplet contains a Dirac fermion and two complex (or 4 real) scalars. In $\mathrm{SQCD}_{5}, n_{c}^{2}-1$ vectors form an adjoint representation of $S U\left(n_{c}\right)$ gauge group while $n_{f} \times n_{c}$ hypers form $n_{f}$ fundamental representations $\mathbf{n}_{\mathbf{c}}$. Note that there are no separate quark and antiquark multiplets; instead, a single $\mathbf{n}_{\mathbf{c}}$ of hypermultiplets contains both the quark and the antiquark (as well as two squarks and two antisquarks). All Yukawa and scalar couplings of a $5 \mathrm{D} \mathcal{N}=1$ theory are related by SUSY to the gauge coupling $g_{5}$; unlike in $4 \mathrm{D}$, there is no independent superpotential. Instead, in 5D there are Chern-Simons interactions of gauge fields and their superpartners,

$$
\begin{aligned}
& \mathcal{L}_{\mathrm{CS}}= \frac{i k_{\mathrm{cs}}}{24 \pi^{2}} \operatorname{tr}\left(A \wedge F \wedge F-\frac{i}{2} A \wedge A \wedge A \wedge F-\frac{1}{10} A \wedge A \wedge A \wedge A \wedge A\right) \\
&+\frac{k_{\mathrm{cS}}}{8 \pi^{2}} \operatorname{tr}\left(\Phi F_{\mu \nu} F^{\mu \nu}\right)+\text { fermionic terms }
\end{aligned}
$$

where $\Phi$ is the adjoint scalar field. To assure gauge invariance of the path integral, the coefficient $k_{\mathrm{cs}}$ (also known as the Chern-Simons level) is quantized: in $\mathrm{SYM}_{5}$ or $\mathrm{SQCD}_{5}$ 
with an even number of flavors, $k_{\mathrm{cs}}$ must be integer; $\mathrm{SQCD}_{5}$ with an odd $n_{f}$ needs a halfinteger $k_{\mathrm{cS}}[1]$.

The vacuum states of $5 \mathrm{D} \mathcal{N}=1$ theories form continuous families parametrized by two kinds of moduli: the Coulomb moduli control the adjoint scalar VEV $\langle\Phi\rangle$ while the Higgs moduli control the squark VEVs. Because these scalars belong to different kinds of supermultiplets (vector versus hyper), the two kinds of moduli do not intermix. That is, the local geometry of the moduli space factorizes into separate Coulomb and Higgs subspaces. The global geometry is more complicated because non-zero squark VEVs require some tuning of the Coulomb moduli and quark masses $m_{f}$. Consequently, the overall moduli space has several branches, each with its own Higgs and Coulomb subspaces: the Coulomb branch with $n_{c}-1$ independent Coulomb moduli but no Higgs moduli at all; the mesonic branches where some Coulomb moduli are fixed to allow some squark VEVS and hence Higgs moduli; and the baryonic branches where all Coulomb moduli are fixed. But in all branches, the Coulomb and the Higgs moduli are completely separated by SUSY.

Provided the UV completion of the quantum theory is manifestly supersymmetric, the separation between the Higgs and the Coulomb moduli remains exact. Also, there are no quantum corrections - perturbative or non-perturbative - to the classical geometry of the Higgs moduli space. This follows from promoting the gauge coupling to a background field [21]: to do it in a supersymmetric manner, $1 / g_{5}^{2}$ must be the scalar member of a vector multiplet and therefore cannot affect the Higgs space geometry [22]. As to the Coulomb moduli space geometry, the quantum corrections stop at the one-loop level. In terms of the prepotential,

$$
\mathcal{F}=\mathcal{F}_{\text {tree }}+\mathcal{F}_{1-\text { loop }}, \quad \text { exactly, }
$$

and there are no further perturbative or non-perturbative corrections [1]. For $\mathrm{SQCD}_{5}$,

$$
\begin{aligned}
\mathcal{F}_{\text {tree }}\left(\phi_{1} \ldots, \phi_{n_{c}}\right) & =\frac{1}{2 g_{5}^{2}} \sum_{i=1}^{n_{c}} \phi_{i}^{2}+\frac{k_{\mathrm{cs}}}{48 \pi^{2}} \sum_{i=1}^{n_{c}} \phi^{3} \\
\text { and } \quad \mathcal{F}_{1-\text { loop }}\left(\phi_{1} \ldots, \phi_{n_{c}}\right) & =\frac{1}{96 \pi^{2}} \sum_{i, j=1}^{n_{c}}\left|\phi_{i}-\phi_{j}\right|^{3}-\frac{1}{96 \pi^{2}} \sum_{i=1}^{n_{c}} \sum_{f=1}^{n_{f}}\left|\phi_{i}-m_{f}\right|^{3},
\end{aligned}
$$

where the Coulomb moduli $\phi_{1} \ldots, \phi_{n_{c}}$ are eigenvalues of the adjoint scalar's VEV $\langle\Phi\rangle$. For 
generic values of these moduli the gauge group $S U\left(n_{c}\right)$ is broken to its Cartan subgroup $U(1)^{n_{c}-1}$, and the gauge coupling matrix for the abelian fields follows from the prepotential according to

$$
\left[\frac{1}{g_{5}^{2}}\right]_{i j}=\left(\begin{array}{c}
\text { moduli } \\
\text { metric }
\end{array}\right)_{i j}=\frac{\partial^{2} \mathcal{F}}{\partial \phi_{i} \partial \phi_{j}} .
$$

This matrix must be positive-definite for all values of the moduli $\phi_{i}$, which restricts the discrete parameters of $\mathrm{SQCD}_{5}$ to [2]

$$
\begin{aligned}
\left|k_{\mathrm{cs}}\right|+\frac{n_{f}}{2} & \leq n_{c} \quad \text { for } n_{c} \geq 3 \\
n_{f} & \leq 7 \quad \text { for } n_{c}=2
\end{aligned}
$$

The abelian gauge fields have Chern-Simons interactions with each other:

$$
\mathcal{L} \supset \sum_{i j k} \frac{K_{i j k}}{48 \pi^{2}} A_{i} \wedge F_{j} \wedge F_{k} \quad \text { where } \quad K_{i j k}=\frac{\partial^{3} \mathcal{F}}{\partial \phi_{i} \partial \phi_{j} \partial \phi_{k}}
$$

Gauge invariance requires the coefficients $K_{i j k}$ to be integer, and this restricts the prepotential so much that there are no quantum corrections beyond the one-loop level.

Finally, a point of terminology. In this paper, we distinguish between the non-dynamical parameters of the $5 \mathrm{D}$ theory such as $1 / g_{5}^{2}$ and quark masses and the dynamical moduli of its vacua such as $\phi_{i}$ or the Higgs moduli of squark VEVs. However, the phase diagram of the theory involves both the parameters and the moduli; for example, in $\S 6.1$ we shall see that an $S U(2)$ SYM theory has a phase transition at $\left(8 \pi^{2} / g_{5}^{2}\right)=-\phi$ rather than at $\left(8 \pi^{2} / g_{5}^{2}\right)=0$. Consequently, when appropriate we shall put the parameters and the moduli into a combined parameter/moduli space.

\section{Overview of Deconstruction:}

And now we turn to the dimensional deconstruction. Most generally, the deconstruction relates simple gauge theories in spaces of higher dimension to more complicated theories in fewer dimensions of space: The extra dimensions of space are 'deconstructed' into quiver diagrams of the 'theory space' $[15,16]$. In simple cases, deconstruction is a three-step procedure: First, one discretizes the extra dimension(s) - say, the $x^{4}$ space coordinate of a $4+1$ dimensional theory - into a lattice of small but finite spacing $a$. On this lattice, 
the $A_{0,1,2,3}$ components of the gauge field reside on lattice nodes, while the $A_{4}$ component is realized via unitary matrices $U_{\ell}=$ path-ordered $\exp \left(i \int_{a \ell}^{a(\ell+1)} A_{4} d x^{4}\right)$ residing on links. Second, one reinterprets the lattice as a quiver diagram describing a complicated 4D field theory with a large number of gauge group factors (one per lattice site) with equal couplings $g_{4}^{(\ell)} \equiv g_{4}=g_{5} / \sqrt{a}$. The link variables $U_{\ell}$ become $4 \mathrm{D}$ non-linear sigma models $\Omega_{\ell}$ with $F_{\pi}=1 /\left(a g_{4}\right)$ and transforming in bi-fundamental representations $\left(\square_{\ell}, \square_{\ell+1}\right)$ of the gauge group $\prod_{\ell} S U(n)_{\ell}$. Finally, one adds degrees of freedom to make the theory renormalizable in $4 \mathrm{D}$; this includes promoting non-linear sigma models $\Omega_{\ell}$ to linear sigma models, or perhaps realizing them as techni-pions of some kind of technicolor (with a separate technicolor group for each $\Omega_{\ell}$ ). The resulting $4 \mathrm{D}$ gauge theory can often be summarized by a quiver diagram - hence the name quiver theory - for example

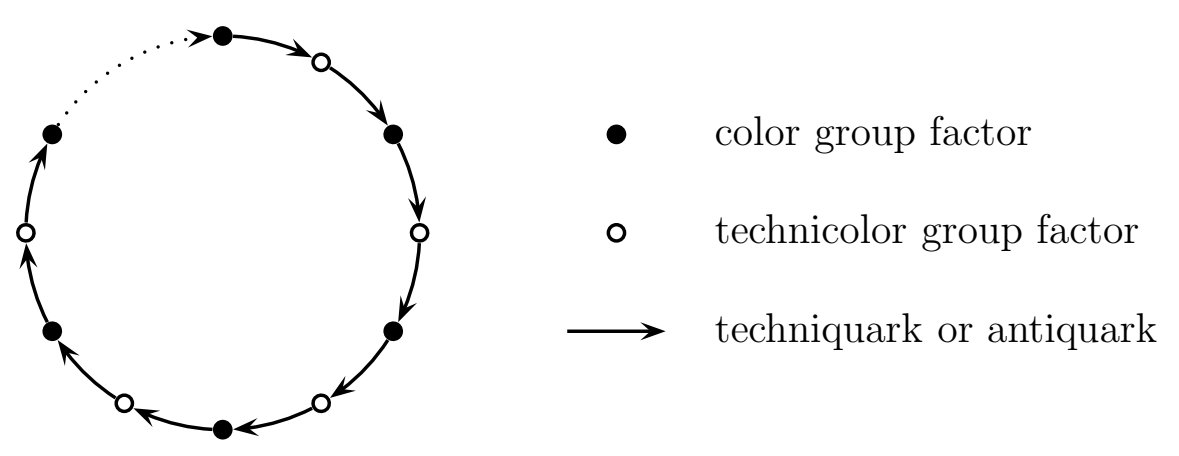

for the 5D YM theory deconstructed in [15]. In order to have a finite number of 4D fields, the quiver should have a finite size $L-$ in $5 \mathrm{D}$ terms, this means that the deconstructed dimension $x^{4}$ is also compactified on a large circle of length $2 \pi R=L a$ - but eventually one may take the $L \rightarrow \infty$ limit and recover the uncompactified 5D physics. In this limit, the lattice spacing $a$ remains finite and serves as the UV regulator: For energies $E \ll(1 / a)$ the physics is $5 \mathrm{D}$ but for $E \gtrsim(1 / a)$ it becomes $4 \mathrm{D}$.

Dimensional deconstruction of supersymmetric theories breaks half of supercharges for every discretized dimension [19,23]; in particular, for $\mathcal{N}=1$ theories in $5 \mathrm{D}$, deconstructing one dimension breaks 4 out of 8 supercharges. Fortunately, the 4 supercharges which remain unbroken act as custodial symmetries of the complete 5D SUSY in the low-energy effective theory. Indeed, for $E \ll(1 / a)$ the fifth dimension is effectively continuous, and if the effective $5 \mathrm{D}$ theory happen to have $S O(4,1)$ Lorentz symmetry as well as any SUSY at all, then it must have the complete 5D SUSY algebra with all 8 supercharges. This is not as easy as it 
sounds because the $S O(4,1)$ Lorentz symmetry of the continuum limit is far from automatic in partially latticized theories. Instead, one often needs to fine-tune the lattice action (i.e., the $4 \mathrm{D}$ Lagrangian of the quiver theory) to make sure that all light particle species have the same light speed in the discretized direction. However, once this is achieved, the recovery of all 8 supercharges in the 5D continuum limit is automatic [19].

From the 4D point of view, the quiver theory has 4 exact supercharges, which means that some properties of the theory are holomorphic and can be calculated exactly, including all the non-perturbative effects. Such properties include the phase structure of the theory, and also moduli dependence of gauge couplings for the massless vector fields. The basic idea of quantum deconstruction is to interpret these data in 5D terms; this allows practical use of the dimensional deconstruction as a UV completion of quantum 5D theories.

\section{Outline:}

This article is organized as follow. In the next section $(\S 2)$ we deconstruct $\mathrm{SQCD}_{5}$ with quarks at the semiclassical level of analysis. Instead of following the 3 -step procedure outlined above - discretize, re-interpret, and make renormalizable - we work in reverse. That is, we start with a quiver diagram, build a corresponding $4 \mathrm{D}, \mathcal{N}=1,\left[S U\left(n_{c}\right)\right]^{L}$ gauge theory, and then show that it indeed deconstructs the 5D SQCD. Specifically, we verify that the classical vacua of the $4 \mathrm{D}$ quiver theory correspond to the classical vacua of the $5 \mathrm{D}$ SQCD, and for each vacuum, the spectrum of light $4 \mathrm{D}$ particles agrees with the Kaluza-Klein reduction (on a latticized circle of length $2 \pi R=L a$ ) of the $5 \mathrm{D}$ gluons, quarks and their superpartners.

In $\S 3$ we deconstruct the Chern-Simons interactions of the 5D gauge fields. We show how to control the Chern-Simons level $k_{\mathrm{cs}}$ by adding extra quark flavors to the $4 \mathrm{D}$ theory. The extra flavors do not have any light modes and thus do not deconstruct any 5D particles; instead, they decouple at the $5 \mathrm{D}$ threshold $E=(1 / a)$. But integrating out those quarks leaves behind quantum corrections to the low-energy Lagrangian; in 5D terms, such corrections lower $k_{\mathrm{cs}}$ by the number $\Delta F$ of extra flavors. Altogether, we end up with $F=n_{f}+\Delta F$ $4 \mathrm{D}$ flavors where $n_{f}$ is the number of $5 \mathrm{D}$ flavors, and $\Delta F$ is used to set

$$
k_{\mathrm{cs}}=n_{c}-\frac{n_{f}}{2}-\Delta F .
$$

Interestingly, the values of $k_{\mathrm{cs}}$ which may be deconstructed in this way are precisely the 
values allowed by eq. (1.6).

In $\S 4$ we deconstruct the $5 \mathrm{D}$ gauge coupling and their moduli dependence. We start with the Coulomb phase of the $4 \mathrm{D}$ quiver where the massless gauge bosons belong to $[U(1)]^{n_{c}-1} \subset$ $\operatorname{diag}\left[S U\left(n_{c}\right)^{L}\right][24]$; the couplings of these abelian bosons are encoded in a hyperelliptic spectral curve, which was computed in [25]. ${ }^{\langle 1\rangle}$ This curve has moduli and parameters, and our first task is to map them to 5D moduli $\phi_{i}$ and parameters $m_{f}$ and $h=\left(8 \pi^{2} / g_{5}^{2}\right)$. Then, we take the decompactification limit $L \rightarrow \infty$ while all the moduli and parameters remain fixed. In this limit, the spectral curve simplifies (as long as $g_{5}$ is weak enough), which helps us to evaluate the abelian gauge coupling matrix $\tau_{i j}$. We find that $\operatorname{Im} \tau_{i j}=L a \times$ a finite matrix $\left[4 \pi g_{5}^{-2}(\phi)\right]_{i j}$, which we interpret as the deconstructed $5 \mathrm{D}$ abelian coupling matrix; this corresponds to $4 \mathrm{D}$ massless fields being zero modes of the $5 \mathrm{D}$ fields compactified on a circle of length $2 \pi R=L a$.

Although the 4D quiver theory has only 4 supercharges, the deconstructed 5D gauge couplings are consistent with a prepotential, which confirms SUSY enhancement to 8 supercharges in the continuum 5D limit. Moreover, the prepotential turns out exactly as in eqs. (1.2-4), which shows that dimensional deconstruction indeed works at the quantum level: the 5D loop corrections follow from loop and instantonic corrections in 4D (see also $[23])$.

The deconstructed tree-level 5D coupling $h=\left(8 \pi^{2} / g_{5}^{2}\right)$ is also affected by the 4D quantum corrections. We find that the allowed range of $h$ depends on the Chern-Simons level of the deconstructed $\mathrm{SQCD}_{5}$ : For $k_{\mathrm{cs}}= \pm\left(n_{c}-\frac{1}{2} n_{f}\right), h$ runs from $+\infty$ down to a finite lower limit $h_{\text {min }}= \pm \frac{1}{2} \sum_{f} m_{f}$; but for other values of $k_{\mathrm{cs}}$ there is no lower limit and $h$ can take any value between $+\infty$ and $-\infty$. The negative values of $h$ do not make sense in terms of ordinary $\mathrm{SQCD}_{5}$; instead, they corresponds to exotic strongly-coupled phases of the 5D theory $[2,8]$.

In $\S 5$, we discuss quantum corrections to baryonic Higgs branches of the deconstructed $\mathrm{SQCD}_{5}$. (The corrections are to the Coulomb moduli and parameters of such branches rather than to the Higgs moduli.) We also find that $5 \mathrm{D}$ theories with $\left|k_{\mathrm{cs}}\right| \leq\left(n_{f} / 2\right)$ have

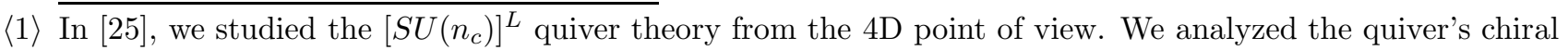
ring, which summarizes its exactly calculable holomorphic data. In this paper, we use these data to obtain the $5 \mathrm{D}$ properties of the deconstructed $\mathrm{SQCD}_{5}$. 
exotic Higgs branches at strong coupling: the $h$ parameter must be fixed at $h=h_{b}$ where $h_{b}=O\left(m_{f}\right) \ll(1 / a)$. In particular, SYM theories $\left(n_{f}=0\right)$ with $k_{\mathrm{cs}}=0$ have exotic Higgs branches at $h=0$. The physical nature of the exotic Higgs branches is unclear from the $5 \mathrm{D}$ point of view, but in $4 \mathrm{D}$ they are simply baryonic branches which involve some of the $\Delta F$ extra quark flavors. At weak coupling, these quarks are heavy (mass $=O(1 / a)$ ) and decouple from the 5D physics, but in the strongly coupled quiver they develop zero modes, hence a baryonic branch for $h=h_{b} \ll(1 / a)$. String/M implementations of 5D SYM with $\left|k_{\mathrm{cs}}\right| \leq\left(n_{f} / 2\right)$ have similar exotic Higgs branches [5] at fixed $h=h_{b}\left(m_{f}\right)$, and alas their physical nature is just as unclear from the 5D QFT point of view as in dimensional deconstruction.

In $\S 6$ we present four examples of deconstructed $\mathrm{SQCD}_{5}$ theories and study their $h<0$ phases. For $h<0$, the $4 \mathrm{D}$ theory is strongly coupled at the $4 \mathrm{D} \rightarrow 5 \mathrm{D}$ threshold $E=(1 / a)$, but thanks to unbroken $\mathcal{N}=1$ SUSY, the spectral curve of the quiver is exactly calculable despite the strong coupling. This allows us to deconstruct the $h<0$ regime of the 5D theory just as easily as the weakly-coupled $h>0$ regime. Sometimes, the two regimes are separated by a phase transition: Although in $4 \mathrm{D}$ there is only one Coulomb phase because the spectral curve is holomorphic, the decompactification limit $L \rightarrow \infty$ of the spectral curve may be different for $h<0$ than for $h>0$, and that leads to different phases in 5D.

For simplicity, all our examples have $n_{c}=2$. The first example ( $\left.\S 6.1\right)$ has $n_{f}=0$ and $\Delta F=1$ while the second example $(\S 6.2)$ has $n_{f}=0$ and $\Delta F=2$. For $h>0$, they deconstruct SYM theories with different $5 \mathrm{D}$ vacuum angles $(\theta=\pi$ for $\Delta F=1$ and $\theta=0$ for $\Delta F=2)$. But the $h<0$ regimes of the two models are very different: the $\Delta F=1$ model has two distinct Coulomb phases - the SYM phase and the $E_{0}$ phase — separated by a flop transition, while the $\Delta F=2$ model has only one Coulomb phase, but it also has an exotic Higgs branch.

In $\S 6.3$ we present two more $S U(2)$ models, this time with $n_{f}=2$ and $\Delta F=0$ or 1 . For simplicity, we restrict our analysis to equal quark masses (modulo sign) for the two 5D flavors. Nevertheless, we find several distinct phases, both Coulomb and Higgs.

All 4 models are presented in much detail, which makes for a rather looong section $\S 6$. But the main result can be stated in once sentence: in all examples, the deconstructed $\mathrm{SQCD}_{5}$ 
has exactly the same phase diagram as the string-theoretical UV completion of the same $5 D$ theory. The readers who are not interested in technical details may skim this section and focus only on the phase diagrams themselves: they appear on pages $59,67,75-76,82,85$, and 87 .

Finally, in $\S 7$ we show that for all $n_{c}, n_{f}$, and $k_{\mathrm{cs}}$, the deconstructed $\mathrm{SQCD}_{5}$ and the string-theoretical implementation of the same 5D theory via a brane web are always in the same universality class. In particular, they always have similar phase diagrams and similar prepotentials $\mathcal{F}\left(\phi_{1}, \ldots, \phi_{n_{c}} ; h ; m_{1}, \ldots, m_{n_{f}}\right)$. However, the two UV completions are not dual to each other and become dissimilar outside of the zero-energy limit.

Instead, the $5 \mathrm{D}$ universality between deconstruction and brane webs is similar to the $4 \mathrm{D}$ universality between SQCD and MQCD [26, 27, 28]. In fact, our proof is based on the 4D universality: We start with deconstructed $\mathrm{SQCD}_{5}$, treat it as a $4 \mathrm{D}\left[S U\left(n_{c}\right)\right]^{L}$ quiver theory, and take its M-theory counterpart - the M5 brane spanning the 4D Minkowski space times the quiver's spectral curve. This M theory is not dual to the deconstructed $\mathrm{SQCD}_{5}$, but it's in the same universality class.

And then we show that the $L a \rightarrow \infty$ limit of the M5 brane is dual to a type IIB $(p, q)$ 5-brane web, and moreover this web implements the very $\mathrm{SQCD}_{5}$ we have started from. And since duality implies universality (but not the other way around), it follows that the brane web is in the same universality class as the deconstructed theory; but they are not dual to each other.

To summarise, in this paper we show how to use dimensional deconstruction as a UV completion of a 5D SUSY gauge theory such as $\mathrm{SQCD}_{5}$. We show how to extract 5D quantum effects such as loop corrections to the prepotential from the 4D loop and instantonic effects - which can be calculated exactly thanks to the unbroken $\mathcal{N}=1$ SUSY in 4 D. We show how to deconstruct the 5D phase diagrams, including the non-classical $h<0$ phases. And we show that at the end of the day, the dimensional deconstruction is in the same universality class as string-theoretical UV completions of the same 5D theory. 


\section{Semiclassical (De) Construction}

In this section, we perform a reverse deconstruction - at the semiclassical level of analysis - of $\mathrm{SQCD}_{5}$ with arbitrary numbers of colors and flavors. That is, we start with a quiver diagram, build a $4 \mathrm{D} \mathcal{N}=1$ gauge theory, and then show that it indeed deconstructs the 5D SQCD. Specifically, we verify that the classical vacua of the 4D quiver theory correspond to the classical vacua of the $5 \mathrm{D}$ SQCD, and for each vacuum, the spectrum of light $4 \mathrm{D}$ particles agrees with the Kaluza-Klein reduction (on a latticized circle of length $2 \pi R=L a$ ) of the 5D gluons, quarks and their superpartners.

We start with quiver diagrams of general form

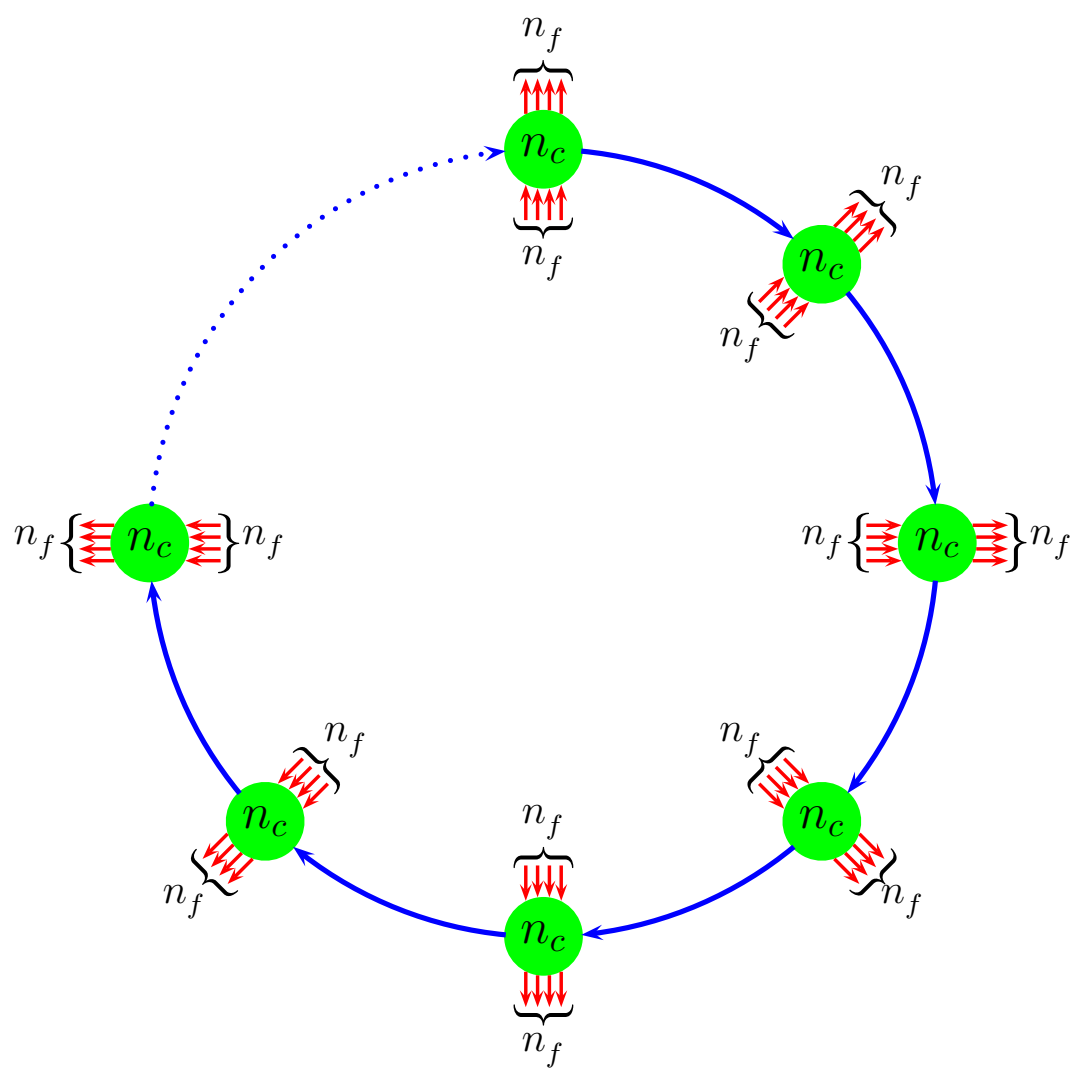

Physically, each green circle of this diagram corresponds to a simple $S U\left(n_{c}\right)_{\ell}$ factor of the net $4 \mathrm{D}$ gauge group

$$
G_{4 \mathrm{D}}=\prod_{\ell=1}^{L} S U\left(n_{c}\right)_{\ell}
$$


while the red and blue arrows denote the chiral superfields:

$$
\begin{aligned}
& \rightrightarrows \text { 引 quarks } Q_{\ell}^{f}=\left(\square_{\ell}\right), \quad f=1,2, \ldots, n_{f}, \\
& \left.\sum\right\} \text { antiquarks } \widetilde{Q}_{\ell}^{f}=\left(\bar{\square}_{\ell}\right), \quad f=1,2, \ldots, n_{f},
\end{aligned}
$$

$\longrightarrow$ bi-fundamental link fields $\Omega_{\ell}=\left(\square_{\ell+1}, \bar{\square}_{\ell}\right)$,

where $\ell=1,2, \ldots, L$ is understood modulo quiver size $L$. From the $4 \mathrm{D}$ point of view, $L$ is a fixed parameter of the theory, but for the deconstruction purposes we must later take the $L \rightarrow \infty$ limit in order to recover the un-compactified 5D physics.

Similar to many other deconstructed theories, the quiver (2.1) can be obtained by orbifolding a 4D theory with a much larger but simple gauge group and higher SUSY, namely $\mathcal{N}=2 \mathrm{SQCD}$ with $L \times n_{c}$ colors (but only $n_{f}$ flavors) [29, 30]: A $\mathbb{Z}_{L}$ twist removes the extra supercharges and reduces the gauge symmetry from $S U\left(L \times n_{c}\right)$ down to

$$
S\left(\left[U\left(n_{c}\right)\right]^{L}\right)=\left[S U\left(n_{c}\right)\right]^{L} \times[U(1)]^{L-1} .
$$

However, the abelian photons of the orbifold theory suffer from triangular anomalies and therefore must be removed from the effective low-energy theory. In string orbifolds such removal is usually accomplished via the Green-Schwarz terms [31, 32], but at the field theory level we simply discard the abelian factors of the orbifolded symmetry (2.4) and interpret the nodes (green circles) of the quiver diagram (2.1) as purely non-abelian $S U\left(n_{c}\right)_{\ell}$ factors.

To complete the 4D quiver theory we must define its tree-level couplings. The orbifolding procedure gives us two types of couplings inherited from the 'original' $\mathcal{N}=2 \mathrm{SQCD}$, namely the same gauge coupling $g_{\ell} \equiv g$ for all the $S U\left(n_{c}\right)_{\ell}$ factors of the quiver, and the Yukawa coupling $\gamma$ of the "hopping" superpotential

$$
W_{\mathrm{hop}}=\gamma \sum_{\ell=1}^{L} \sum_{f=1}^{n_{f}}\left(\widetilde{Q}_{\ell+1}^{f} \Omega_{\ell} Q_{\ell}^{f}-\mu_{f} \widetilde{Q}_{\ell}^{f} Q_{\ell}^{f}\right)
$$

which makes the quark fields propagate in the discretized $x^{4}$ direction. Classically $\gamma=g$ because of $\mathcal{N}=2$ SUSY of the "mother theory"; in lattice terms, this equality assures that the quarks and the gluons have equal light speeds. Besides the couplings, we also have quark 
masses $\mu_{f}$. Formally, we may derive them from the orbifolded 5D quark masses, but we shall see momentarily that the relation between the $4 \mathrm{D}$ and the $5 \mathrm{D}$ quark masses of the deconstructed theory is more complicated.

Finally, to make the deconstruction work, we need the O'Raifeartaigh superpotential

$$
W_{\Sigma}=\beta \sum_{\ell=1}^{L} s_{\ell} \times\left(\operatorname{det}\left(\Omega_{\ell}\right)-v^{n_{c}}\right)
$$

where $s_{\ell}$ are singlet fields (one for each $\ell=1,2, \ldots, L$ ) not shown on the quiver diagram (2.1). These singlets and the O'Raifeartaigh terms (2.6) do not follow from the orbifolding: We simply add them by hand at the same time as we remove the abelian factors from the orbifolded gauge group (2.4). The purpose of this modification is to turn each bi-linear link field $\Omega_{\ell}$ into an $S L\left(n_{c}, \mathbb{C}\right)$ linear sigma model where on-shell

$$
\operatorname{det}\left(\Omega_{\ell}\right) \equiv v^{n_{c}}=\text { const. }
$$

Note that $S L\left(n_{c}, \mathbb{C}\right)$ is the complexified $S U\left(n_{c}\right)$ group manifold, and this is precisely what we want for the link variables of a supersymmetric $S U\left(n_{c}\right)$ gauge theory on the lattice. In $5 \mathrm{D}$ terms,

$$
\Omega_{\ell}(x)=v \times \exp _{\substack{\text { Path } \\ \text { ordered }}}\left(\int_{a \ell}^{a(\ell+1)} d x^{4}\left(i A_{4}(x)+\phi(x)\right)\right)+\text { fermionic terms }
$$

where $\phi(x)$ is the scalar superpartner of the $5 \mathrm{D}$ vector field $A_{\mu}(x), \mu=0,1,2,3,4$.

Having defined the 4D quiver theory, we must now verify that it indeed deconstructs the 5D SQCD. At the semi-classical level of analysis of this section, this means verifying that: (A) the vacuum field configurations of the 5D and the 4D theory correspond to each other according to the field map (2.8), and (B) for each vacuum, the spectrum of light 4D particles follows from the Kaluza-Klein reduction of the 5D particles on a latticized circle of length $2 \pi R=L a[15,16]$. That is, for each $5 \mathrm{D}$ quark or gluon (or a superpartner) we must have a 
series of $L 4 \mathrm{D}$ particles with similar quantum numbers and $4 \mathrm{D}$ masses given by

$$
M_{4}^{2}=m_{5}^{2}+P_{4}^{2}+O\left(a m_{5}^{3}, a^{2} P_{4}^{4}, \ldots\right)
$$

where

$$
P_{4}=\frac{2 \pi k}{L a}+p_{0}, \quad k=0,1,2, \ldots \bmod L
$$

is the quantized momentum in the $x^{4}$ direction and the quantization shift $p_{0}$ allows for Wilson lines, etc. Furthermore, all the light $4 \mathrm{D}$ particles $\left(M_{4} \ll(1 / a)\right)$ must belong to such Kaluza-Klein series, although the heavy $4 \mathrm{D}$ particles $\left(M_{4} \gtrsim(1 / a)\right)$ do not have to have 5D counterparts.

We begin with the simplest $5 \mathrm{D}$ vacuum state with unbroken $S U\left(n_{c}\right)$ where all the gluons are massless while the quarks have their bare masses $m_{f}$. According to the field map (2.8), $\langle\phi\rangle=\left\langle A_{4}\right\rangle=0$ in $5 \mathrm{D}$ translates to the $4 \mathrm{D}$ link field VEVs

$$
\left\langle\Omega_{\ell}\right\rangle \equiv v \times \mathbf{1}_{n_{c} \times n_{c}}
$$

which break the $4 \mathrm{D}$ gauge symmetry (2.2) down to the 'diagonal'

$$
S U\left(n_{c}\right)_{\operatorname{diag}}=\operatorname{diag}\left[\prod_{\ell} S U\left(n_{c}\right)_{\ell}\right]
$$

while the rest of the $4 \mathrm{D}$ vector fields acquire masses

$$
M_{4}^{2}(k)=g^{2}|v|^{2} \times 4 \sin ^{2} \frac{\pi k}{L} .
$$

This spectrum indeed matches eq. (2.9) for $m_{5}=0$, provided we identify the lattice spacing as

$$
a=\frac{1}{g|v|}
$$

Similarly, the quark mass spectrum also has deconstructive form for flavors with bare $4 D$ masses $\mu_{f}$ near $v$. Indeed, consider the mass matrix for the quarks $Q_{\ell}^{f}$ and the antiquarks 
$\widetilde{Q}_{\ell}^{f}$ of a fixed flavor $f$ but all $\ell=1, \ldots, L$ : The exact eigenvalues of this matrix are given by

$$
M_{4}^{2}(k)=|\gamma|^{2} \times\left|v e^{2 \pi i k / L}-\mu_{f}\right|^{2}, \quad k=1, \ldots, L,
$$

and for $\mu_{f}$ near $v$, this spectrum does have deconstructive form (2.9) where

$$
\begin{aligned}
m_{5} & =\left|\mu_{f}\right|-|v|, \\
p_{0} & =|\gamma v| \times \arg \left(\mu_{f} / v\right), \\
\text { and } a & =\frac{1}{|\gamma v|} .
\end{aligned}
$$

Clearly, in order to satisfy both eqs. (2.14) and (2.18) we must have equal gauge and Yukawa couplings $g=|\gamma|$. In a quantum theory, this means fine-tuning the non-holomorphic Kähler parameters of the quiver theory such that the renormalized physical couplings satisfy

$$
g_{\text {phys }}=|\gamma|_{\text {phys }} \text { exactly, }
$$

or in non-perturbative terms, in the very low energy limit $E \ll v / L$, the effective theory (the diagonal $S U\left(n_{c}\right)$ with an adjoint field $\Phi$ and several quark flavors) should be $\mathcal{N}=2$ supersymmetric. Without this condition, the deconstructed theory would have quarks and gluons with different effective speeds of light in the $x^{4}$ direction. This is a common problem in lattice theories with some continuous dimensions (eg. Hamiltonian lattice theories with continuous time but discrete space), and the common solution is fine-tuning of the lattice parameters. For the problem at hand, the fine tuning of the quiver theory involves the Kähler parameters (such as coefficients of the quarks' and antiquarks' kinetic-energy Lagrangian terms) and does not affect any of the holomorphic properties of the quiver such as its chiral ring. Consequently, in the following we shall simply assume that the couplings are fine-tuned according to eq. (2.19) and focus on other issues.

Next, consider the Coulomb branch of the $\mathrm{SQCD}_{5}$ moduli space where the squarks have zero VEVs but $\langle\phi\rangle \neq 0$. Generically, the $\langle\phi\rangle$ matrix has $n_{c}$ distinct eigenvalues $\left(\phi_{1}, \ldots, \phi_{n_{c}}\right)$, the $S U\left(n_{c}\right)$ gauge symmetry is broken down to its Cartan subgroup $(U(1))^{n_{c}-1}$ and the 
off-diagonal gluons $G_{i j}$ have masses

$$
m_{5}\left[G_{i j}\right]=\phi_{i}-\phi_{j}
$$

At the same time, the quarks have color- and flavor-dependent masses

$$
m_{5}\left[q^{i, f}\right]=m_{f}-\phi_{i}
$$

Similarly, the 4D quiver theory has Coulomb branch vacua with zero squark VEVs but nontrivial link VEVs $\left\langle\Omega_{\ell}\right\rangle \neq v \times 1_{n_{c} \times n_{c}}$. Combining the D-term constraints for all the $S U\left(n_{c}\right)_{\ell}$ gauge groups

$$
\Omega_{\ell}^{\dagger} \Omega_{\ell}-\Omega_{\ell-1} \Omega_{\ell-1}^{\dagger} \propto \mathbf{1}_{n_{c} \times n_{c}} \forall \ell
$$

with the F-term constraints (2.7), we find that all the $\left\langle\Omega_{\ell}\right\rangle$ matrices must be equal to each other modulo an $\ell$-dependent gauge transform. Moreover, we may diagonalize all the matrices at once and set

$$
\forall \ell:\left\langle\Omega_{\ell}\right\rangle=v \times \operatorname{diag}\left(e^{a \varphi_{1}}, e^{a \varphi_{2}}, \ldots, e^{a \varphi_{n_{c}}}\right)
$$

for some complex numbers $\left(\varphi_{1}, \varphi_{2}, \ldots, \varphi_{n_{c}}\right)$ satisfying $\sum_{i} \varphi_{i}=0$. According to the field map (2.8), this corresponds to the 5D VEVs

$$
\langle\phi\rangle+i\left\langle A^{4}\right\rangle=\operatorname{diag}\left(\varphi_{1}, \varphi_{2}, \ldots, \varphi_{n}\right)
$$

i.e., $\phi_{i}=\operatorname{Re} \varphi_{i}$ are the 5D scalar VEVs of the Coulomb branch while the $A_{i}^{4}=\operatorname{Im} \varphi_{i}$ are Wilson lines of the diagonal gauge fields around the compactified $x^{4}$ dimension.

Generically, all the $\varphi_{i}$ are distinct and the 4D gauge symmetry is broken all the way down to the Cartan $(U(1))_{\text {diag }}^{n_{c}-1}$ subgroup of the diagonal $S U\left(n_{c}\right)$. The mass matrix of the remaining $4 \mathrm{D}$ gauge fields has eigenvalues

$$
M_{4}^{2}\left[G_{i j}^{(k)}\right]=g^{2}|v|^{2} \times\left|e^{2 \pi i k / L} e^{a \varphi_{i}}-e^{a \varphi_{j}}\right|^{2}
$$

and it is easy to see that for $\phi_{i}, \phi_{j} \ll(1 / a)$ and $|k| \ll L$ this $4 \mathrm{D}$ spectrum has the decon- 
structed form (2.9) where the lattice spacing $a$ is exactly as in eq. (2.14), the 5D mass

$$
m_{5}\left[G_{i j}\right]=\operatorname{Re}\left(\varphi_{i}-\varphi_{j}\right) \equiv \phi_{i}-\phi_{j}
$$

is in perfect agreement with the $5 \mathrm{D}$ formula $(2.20)$, and the $P_{4}$ quantization shift $p_{0}$ is precisely the appropriate Wilson line

$$
p_{0}\left[G_{i j}\right]=\operatorname{Im}\left(\varphi_{i}-\varphi_{j}\right) \equiv A_{i}^{4}-A_{j}^{4}
$$

At the same time, the 4D quarks have mass eigenvalues

$$
M_{4}^{2}\left[Q_{(k)}^{i, f}\right]=|\gamma|^{2} \times\left|v e^{2 \pi i k / L} e^{a \varphi_{i}}-\mu_{f}\right|^{2}=\frac{1}{a^{2}} \times\left|e^{2 \pi i k / L} e^{a \varphi_{i}}-e^{a m_{f}}\right|^{2}
$$

where in the second equality we use eq. (2.18) for the lattice spacing $a$ and define complex $5 \mathrm{D}$ masses $m_{f}$ according to

$$
m_{f} \stackrel{\text { def }}{=} \frac{1}{a} \log \frac{\mu_{f}}{v}
$$

In the deconstruction limit, the real parts $\operatorname{Re}\left(m_{f}\right)$ of these complex masses act as the bare 5D quark masses, while their imaginary parts $\operatorname{Im}\left(m_{f}\right)$ correspond to Wilson lines of the flavor symmetries, cf. eq. (2.17). Indeed, if we restrict to $m_{f} \ll(1 / a)\left(i . e\right.$., $\mu_{f}$ near $\left.v\right), \varphi_{i} \ll(1 / a)$ and $|k| \ll L$, then the quark mass spectrum (2.28) has the deconstructed form (2.9) where the 5D masses and the Wilson lines are exactly as for the Coulomb branch of the $\mathrm{SQCD}_{5}$ :

$$
\begin{aligned}
m_{5}\left[Q^{i, f}\right] & =\operatorname{Re}\left(m_{f}-\varphi_{i}\right)=\operatorname{Re} m_{f}-\phi_{i} \\
p_{0}\left[Q^{i, f}\right] & =\operatorname{Im}\left(m_{f}-\varphi_{i}\right)=\operatorname{Im} m_{f}-A_{i}^{4}
\end{aligned}
$$

Besides the Coulomb branch, the moduli space of $\mathrm{SQCD}_{5}$ has mesonic Higgs branches iff some of the quark masses are degenerate. On such branches, some squark fields have non-zero VEVs, some of the $\phi_{i}$ eigenvalues are fixed, and the surviving gauge theory has reduced rank $r<\left(n_{c}-1\right)$. For example, for $m_{1}=m_{2}$ there is a mesonic Higgs branch with non-zero squark VEVs $\left\langle q_{\alpha}^{i, f}\right\rangle$ for $i=1$ and $f=1,2$; the hypermultiplet components (indexed 
by $\alpha, \beta)$ are constrained by the D-terms to satisfy

$$
D^{t}=\eta_{\alpha \beta}^{t}\left\langle q_{\alpha}^{1,1}\right\rangle\left\langle q_{\beta}^{1,1}\right\rangle^{*}+\eta_{\alpha \beta}^{t}\left\langle q_{\alpha}^{1,2}\right\rangle\left\langle q_{\beta}^{1,2}\right\rangle^{*}=0 \quad \text { for } t=1,2,3
$$

On this branch, the $\phi_{1}$ eigenvalue is frozen at $\phi_{1}=m_{1}=m_{2}$ while the remaining eigenvalues $\phi_{2}, \ldots, \phi_{n_{c}}$ remain free (except for the $\sum_{i} \phi_{i}=0$ constraint); for generic $\phi_{2}, \ldots, \phi_{n_{c}}$ the gauge symmetry is Higgsed down to $(U(1))^{n_{c}-2}$ while the remaining gluons have masses

$$
m_{5}^{2}\left[G_{i j}\right]=\left(\phi_{i}-\phi_{j}\right)^{2}+\left(\delta_{i, 1}+\delta_{j, 1}-\frac{2}{n_{c}} \delta_{i, 1} \delta_{j, 1}\right) \times \frac{g_{5}^{2}}{4} \sum_{f, \alpha}\left|\left\langle q_{\alpha}^{1, f}\right\rangle\right|^{2}
$$

Likewise, the quiver theory has a mesonic Higgs branch whenever $\mu_{f}^{L}=\mu_{f^{\prime}}^{L} \neq 0$. Indeed, let $\mu_{1}^{L}=\mu_{2}^{L} \neq 0$ and let us freeze the $\left(v e^{a \varphi_{1}}\right)^{L}$ link eigenvalue at the same value, or equivalently let

$$
\varphi_{1}=m_{1}+\frac{2 \pi i k_{1}}{L a}=m_{2}+\frac{2 \pi i k_{2}}{L a}
$$

for some integers $k_{1}$ and $k_{2}$. At this point, the scalar potential has flat directions for the squark and antisquark modes

$$
\left\langle Q_{\ell}^{i, f}\right\rangle=e^{2 \pi i k_{f} \ell / L} \times Q^{i, f}, \quad\left\langle\widetilde{Q}_{\ell}^{i, f}\right\rangle=e^{2 \pi i k_{f}(1-\ell) / L} \times \widetilde{Q}^{i, f},
$$

of the color $i=1$ and the flavors $f=1,2$ only, subject to $\mathrm{F}$-term and $\mathrm{D}$-term constraints

$$
\sum_{f} Q^{1, f} \tilde{Q}^{1, f}=0, \quad \sum_{f}\left(\left|Q^{1, f}\right|^{2}-\left|\tilde{Q}^{1, f}\right|^{2}\right)=0
$$

These squark VEVs Higgs the $\left(S U\left(n_{c}\right)\right)^{L}$ gauge symmetry down to the $\left(S U\left(n_{c}-1\right)\right)^{L}$, which is further broken by the link VEVs $\left\langle\Omega_{\ell}\right\rangle$ down to a subgroup of the diagonal $S U\left(n_{c}-1\right)$. For generic values of the un-frozen eigenvalues $\varphi_{2}, \ldots, \varphi_{n_{c}}$, the unbroken gauge symmetry is

$\langle 2\rangle$ In $5 \mathrm{D}, \mathcal{N}=1$ SUSY there are three D terms forming a triplet of the $S U(2)_{R}$ symmetry. Consequently, for any broken gauge symmetry there are three $\mathrm{D}$-term constraints $D^{t}, t=1,2,3$ : The $\eta_{\alpha \beta}^{t}$ matrices in eq. (2.32) represent the action of the $S U(2)_{R}$ symmetry on the hypermultiplet components $q_{\alpha}$. 
$U(1)^{n_{c}-2}$, and all the remaining gauge fields have masses

$$
\begin{aligned}
M_{4}^{2}\left[G_{i j}^{(k)}\right]= & g^{2}|v|^{2} \times\left|e^{2 \pi i k / L} e^{a \varphi_{i}}-e^{a \varphi_{j}}\right|^{2} \\
& +\left(\delta_{i, 1}+\delta_{j, 1}-\frac{2}{n_{c}} \delta_{i, 1} \delta_{j, 1}\right) \times \frac{g^{2}}{2} \sum_{f}\left(\left|Q^{1, f}\right|^{2}+\left|\widetilde{Q}^{1, f}\right|^{2}\right) .
\end{aligned}
$$

Clearly, this mesonic Higgs branch of the quiver theory deconstructs the similar branch of the $\mathrm{SQCD}_{5}$ : Its root (i.e., the point where it connects to the Coulomb branch) is at the same place $\operatorname{Re} \varphi_{1}=\operatorname{Re} m_{1}=\operatorname{Re} m_{2}$, and the $\mathrm{F} / \mathrm{D}$ term constraints (2.36) match the $5 \mathrm{D}$ constraints (2.32) once we repackage

$$
\left(Q, \widetilde{Q}^{\dagger}\right)^{i, f} \mapsto \sqrt{a} q_{\alpha}^{i, f}
$$

(The factor $\sqrt{a}$ here translates between the 4D and the 5D canonical normalizations of the quark fields.) Finally, the 4D mass spectrum (2.37) has the deconstructed form (2.9) where the 5D masses are exactly as in eq. (2.33), provided we translate squark VEVs according to eq. (2.38) and identify the 5D gauge coupling according to the classical deconstruction formula $[15]$

$$
g_{5}^{2}=a g^{2}
$$

Further coincidences among the quark masses of $\mathrm{SQCD}_{5}$ allows for richer mesonic Higgs branches with more squark VEVs, more frozen $\phi_{i}$ eigenvalues $\left(e g ., \phi_{1}=m_{1}=m_{2}, \phi_{2}=\right.$ $m_{3}=m_{4}$ ) and a lower rank of the unbroken gauge symmetry. The $4 \mathrm{D}$ quiver theory with multiple coincidences among $\mu_{f}^{L}$ has similar Higgs branches, and the deconstruction works so similarly to the above that we don't need to repeat the argument.

Instead, let us consider the baryonic Higgs branch which exists for $n_{f} \geq n_{c}$ when $n_{c}$ of the quark masses add to zero, eg., $m_{1}+m_{2}+\cdots m_{n_{c}}=0$. On this branch, the $\phi$ field is completely frozen at $\phi_{i}=m_{i} \forall i=1, \ldots, n_{c}$, all the quarks with $i=f$ develop similar VEVs

$$
\left\langle q_{\alpha}^{i, f}\right\rangle=\delta^{i, f} \times q_{\alpha}
$$

and the gauge symmetry is completely Higgsed down. The particle spectrum comprises a single massless hypermultiplet $q$ (the baryonic modulus), $n_{c}\left(n_{f}-n_{c}\right)$ short hypermultiplets 
$q^{i, f}$ with masses

$$
m_{5}\left[q^{i, f}\right]=\phi_{i}-m_{f}=m_{i}-m_{f} \text { for } f>n_{c} \text { only }
$$

plus $n_{c}^{2}-1$ long vector multiplets with masses

$$
m_{5}^{2}\left[G_{i j}\right]=\frac{1}{2} g_{5}^{2} \sum_{\alpha}\left|q_{\alpha}\right|^{2}+\left(\phi_{i}-\phi_{j}\right)^{2}
$$

The quiver theory also has a baryonic Higgs branch when $n_{f} \geq n_{c}$ and the product of some $n_{c} 4 \mathrm{D}$ masses equals to $v^{n_{c}}$. Indeed, let $\mu_{1}^{L} \times \mu_{2}^{L} \times \cdots \times \mu_{n_{c}}^{L}=v^{L n_{c}}$ or equivalently $m_{1}+m_{2}+\cdots m_{n_{c}}=0$, and let us freeze all the link eigenvalues at

$$
\varphi_{i}=m_{i}+\frac{2 \pi i k_{i}}{L a} \quad \forall i=1,2, \ldots, n_{c}
$$

At this point, the quark mass matrix due to superpotential (2.5) has zero modes for all $i=f$, which allows non-zero VEVs

$$
\left\langle Q_{\ell}^{i, f}\right\rangle=\delta^{i, f} \times e^{2 \pi i k_{i} \ell / L} \times Q^{i}, \quad\left\langle\widetilde{Q}_{\ell}^{i, f}\right\rangle=\delta_{i}^{f} \times e^{2 \pi i k_{i}(1-\ell) / L} \times \widetilde{Q}^{i},
$$

subject to the $\mathrm{D}$ term constraint

$$
\text { same }\left(\left|Q^{i}\right|^{2}-\left|\widetilde{Q}_{i}\right|^{2}\right) \forall i
$$

and the F-term constraint

$$
\frac{\partial W}{\partial \Omega_{\ell}^{i, i}}=\gamma Q^{i} \widetilde{Q}^{i}-\frac{\beta v^{n_{c}}\left\langle s_{\ell}\right\rangle}{v e^{a \varphi_{i}}}=0 .
$$

The simplest solutions to these constraints are either same $Q^{i} \equiv Q \forall i$ and $\widetilde{Q}^{i} \equiv 0$ (baryonic VEVs only) or vice verse same $\widetilde{Q}^{i} \equiv \widetilde{Q} \forall i$ and $Q^{i} \equiv 0$ (antibaryonic VEVs only), but thanks to the singlet fields $s_{\ell}$ enforcing the determinant constraints (2.7), there are other solutions where both baryonic and antibaryonic VEVs are present at the same time while $\left\langle s_{\ell}\right\rangle \equiv s \neq 0$. 
In such solutions

$$
Q^{i} \times \widetilde{Q}^{i}=\text { const } \times e^{-a \varphi_{i}},
$$

and the color dependence of the right hand side goes away in the deconstruction limit of $\varphi_{i}=m_{i} \ll(1 / a)$. Consequently, the squark VEVs become simply

$$
\left\langle Q_{\ell}^{i, f}\right\rangle=\delta^{i, f} \times e^{2 \pi i k_{i} \ell / L} \times Q, \quad\left\langle\widetilde{Q}_{\ell}^{i, f}\right\rangle=\delta^{i, f} \times e^{2 \pi i k_{i}(1-\ell) / L} \times \widetilde{Q}
$$

for some arbitrary pair $(Q, \widetilde{Q})$ of complex VEVs - which obviously deconstruct the 1 hypermultiplet $\mathrm{VEV}\left\langle q_{\alpha}\right\rangle$ of the 5D theory.

Furthermore, all the gauge symmetries of the 4D quiver theory are Higgsed down and the vector multiplets acquire masses

$$
\begin{aligned}
M_{4}^{2}\left[G_{i j}^{(k)}\right] & =g^{2}\left(|Q|^{2}+|\widetilde{Q}|^{2}\right)+g^{2}|v|^{2} \times\left|e^{2 \pi i k / L} e^{a \varphi_{i}}-e^{a \varphi_{j}}\right|^{2} \\
& \approx g^{2}\left(|Q|^{2}+|\widetilde{Q}|^{2}\right)+\left(\phi_{i}-\phi_{j}\right)^{2}+P_{4}^{2}
\end{aligned}
$$

where the approximation on the second line applies in the deconstructive limit of $\varphi_{i}, m_{i}, P_{4} \ll$ $(1 / a)$. These $4 \mathrm{D}$ masses are in obvious agreement with the $5 \mathrm{D}$ vector masses $(2.42)$, so all we need to check is the supermultiplet structure. In $4 \mathrm{D}, \mathcal{N}=1$ SUSY, the Higgs mechanism eats one chiral multiplet for each vector multiplet which becomes massive, thus each $G_{i j}^{(k)}$ vector eats one linear combination of the three chiral multiplets $\Omega_{\ell}^{i j}, Q_{\ell}^{i j}$ and $\widetilde{Q}_{\ell}^{i j}$ with similar charges. Meanwhile, the other two linear combinations of these three multiplets acquire masses via the Yukawa couplings, and thanks to $\gamma=g$ and $\varphi_{i}=m_{i}$, they end up with exactly the same masses (2.49) as the vector fields. Altogether, this gives us complete $\mathcal{N}=2$ massive long multiplets - or equivalently long 5D massive vector multiplets reduced to $4 \mathrm{D}$.

Finally, the remaining chiral fields of the quiver theory comprise a massless pair $(Q, \widetilde{Q})$ which deconstructs the baryonic hypermultiplet modulus of the 5D theory, plus massive quarks and antiquarks with flavors $f>n_{c}$. These quarks have masses

$$
\begin{aligned}
M_{2}^{4}\left[q_{i, f}^{(k)}\right] & =|\gamma V|^{2} \times\left|e^{2 \pi i k / L} e^{a m_{i}}-e^{a m_{f}}\right|^{2} \quad \text { for } f>n_{c} \text { only } \\
& \approx\left(m_{i}-m_{f}\right)^{2}+P_{4}^{2} \quad \text { for } m_{i}, m_{f}, P_{4} \ll(1 / a)
\end{aligned}
$$


and clearly deconstructs the 5D short hypermultiplets $q_{i, f}$ with masses $(2.41)$.

This completes our classical analysis of the deconstructed $\mathrm{SQCD}_{5}$. At the quantum level of the 4D quiver theory, calculating the mass spectra for various vacua of the theory becomes much more difficult - indeed, the state-of-the-art $\mathcal{N}=1$ technology does not allow for exact non-perturbative calculation of physical masses. Instead, our quantum analysis of the deconstructed $\mathrm{SQCD}_{5}$ will focus on the exactly calculable holomorphic properties of the 4D theory such as the moduli dependence of its gauge couplings. We shall return to this issue in section 4 . 


\section{On Chern-Simons Couplings and Extra 4D Flavors}

The quarks we have studied in the previous section were light compared to the lattice cutoff of the deconstructed 5D theory: $\left|m_{f}\right| \ll(1 / a)$, hence according to eq. (2.29) $\mu_{f} \approx v$. From the quiver point of view, there are no $4 \mathrm{D}$ restrictions on the bare quark masses and one may freely add extra flavors with $\mu_{f} \gg v$ or $\mu_{f} \ll v$. However, all such extra flavors are very heavy from the $5 \mathrm{D}$ point of view $\left(\left|m_{f}\right| \gtrsim(1 / a)\right)$ and decouple from the continuum limit of the 5D theory. In fact, the extra flavors with $\mu_{f} \gg v$ decouple from the $4 \mathrm{D}$ quiver theory above the deconstruction threshold, so there is really no point in considering them any further. On the other hand, the extra flavors with $\mu_{f} \ll v$ are very much present at the deconstruction threshold, and even through they ultimately decouple from the low-energy $5 \mathrm{D}$ theory, the 5D couplings receive quantum corrections from integrating out such extra flavors. Specifically, the extra flavors affect the Chern-Simons level of the deconstructed 5D theory:

$$
k_{\mathrm{cs}}=n_{c}-\#\left\{f: \mu_{f} \ll v\right\}-\frac{1}{2} \#\left\{f: \mu_{f} \sim v\right\}
$$

The purpose of the present section is to prove this formula for $n_{c} \geq 3$.

Let us start with a special case where all flavors have $\mu_{f} \equiv 0$ and the quarks $Q_{\ell}$ and antiquarks $\widetilde{Q}_{\ell}$ at the same site $\ell$ uncouple from each other. Hence, there is no interaction between different link fields $\Omega_{\ell}$ via quarks and antiquarks: Each $\Omega_{\ell}$ couples to the specific pair of $Q_{\ell}$ and $\widetilde{Q}_{\ell+1}$, and they don't couple to any other link $\Omega_{\ell^{\prime}}$. Therefore, we may treat each link $\Omega_{\ell}$ as a separate $S U\left(n_{c}\right)$ sigma model and calculate its Wess-Zumino interactions without any concern for the other link fields $\Omega_{\ell^{\prime}}$.

The Wess-Zumino interactions are topological and they depend only on the way the chiral fermion transform under symmetries of the sigma model, so let us consider the fermions which couple to the non-linear scalar field $\Omega_{\ell}$ : In component field formalism,

$\mathcal{L}_{\text {Yukawa }}\left[\Omega_{\ell}\right]=g \operatorname{tr}\left(\Omega_{\ell}^{\dagger} \lambda_{\ell+1} \Psi_{\ell}^{\Omega}-\Psi_{\ell}^{\Omega} \lambda_{\ell} \Omega_{\ell}^{\dagger}\right)+\gamma \operatorname{tr}\left(\Psi_{\ell+1}^{\widetilde{Q}} \Omega_{\ell} \Psi_{\ell}^{Q}\right)+\beta v^{n_{c}} \Psi_{\ell}^{s} \operatorname{tr}\left(\Psi_{\ell}^{\Omega} \Omega_{\ell}^{-1}\right)+$ H. c.,

where $\lambda_{\ell}, \lambda_{\ell+1}$ are the gauginos and the $\Psi_{\ell}^{\Omega}, \Psi_{\ell+1}^{\widetilde{Q}}, \Psi_{\ell}^{Q}$, and $\Psi_{\ell}^{s}$ are the fermionic members 
of the appropriate chiral multiplets; no other fermions couple to the $\Omega_{\ell}$ sigma model. The $\Omega_{\ell}$ sigma model has a chiral symmetry $S U\left(n_{c}\right)_{L} \times S U\left(n_{c}\right)_{R} \equiv S U\left(n_{c}\right)_{\ell+1} \times S U\left(n_{c}\right)_{\ell}$. Under the right-hand symmetry $U \in S U\left(n_{c}\right)_{\ell}$, the scalar field $\Omega_{\ell}$ transforms according to $\Omega_{\ell} \rightarrow$ $\Omega_{\ell} \times U^{\dagger}$ while the fermionic transformation rules follow from the invariance of the Yukawa Lagrangian (3.2). Specifically,

$$
\Psi_{\ell}^{\Omega} \rightarrow \Psi_{\ell}^{\Omega} U^{\dagger}, \quad \lambda_{\ell} \rightarrow U \lambda_{\ell} U^{\dagger}, \quad \lambda_{\ell+1} \rightarrow \lambda_{\ell+1}, \quad \Psi_{\ell+1}^{\widetilde{Q}} \rightarrow \Psi_{\ell+1}^{\widetilde{Q}}, \quad \Psi_{\ell}^{Q} \rightarrow U \Psi_{\ell}^{Q}, \quad \Psi_{\ell}^{s} \rightarrow \Psi_{\ell}^{s}
$$

From the chiral $S U\left(n_{c}\right)_{R} \equiv S U\left(n_{c}\right)_{\ell}$ point of view, the $\Psi_{\ell}^{\Omega}$ amounts to $n_{c}$ species of antiquarks each transforming according to $\tilde{\psi} \rightarrow \tilde{\psi} \times U^{\dagger}$, while the $\Psi_{\ell}^{Q}$ packs $n_{f}$ species of quarks each transforming according to $\psi \rightarrow U \times \psi$. For $n_{c} \neq n_{f}$ this is a chiral transform, hence the Wess-Zumino action

$$
S_{\mathrm{WZ}}=k_{\mathrm{wZ}} \times \int_{\mathbb{R}^{4}} \Omega_{\mathrm{WZ}}\left(\Omega_{\ell}\right)
$$

where $\Omega_{\mathrm{WZ}}$ is the universal Wess-Zumino $4-$ form $^{\langle 3\rangle}$, and

$$
k_{\mathrm{wz}}=n_{c}-n_{f}
$$

As explained in [33, 20], the Wess-Zumino couplings of the link fields deconstruct the Chern-Simons coupling of the 5D gauge fields: Let $\Omega_{\mathrm{WZ}}\left(\Omega_{\ell}, A_{\ell}^{\mu}, A_{\ell+1}^{\mu}\right)$ be a gauged WZ 4form for the link field $\Omega_{\ell}$ and the (4D) gauge fields $A_{\ell}^{\mu}$ and $A_{\ell+1}^{\mu}$ under which it is charged; then in the continuum 5D limit $a \rightarrow 0$,

$$
\sum_{\ell} \int_{\mathbb{R}^{4}} \Omega_{\mathrm{WZ}}\left(\Omega_{\ell}, A_{\ell}^{\mu}, A_{\ell+1}^{\mu}\right)=\int_{\mathbb{R}^{5}} \Omega_{\mathrm{CS}}\left(A_{5 \mathrm{D}}^{\mu}\right)+O(a)
$$

where

$$
\Omega_{\mathrm{CS}}=\frac{i}{24 \pi^{2}} \operatorname{tr}\left(A \wedge F \wedge F-\frac{i}{2} A \wedge A \wedge A \wedge F-\frac{1}{10} A \wedge A \wedge A \wedge A \wedge A\right)
$$

$\langle 3\rangle$ The name "Wess-Zumino form" is often used for the 5 -form $d \Omega_{\mathrm{WZ}}$ rather than the 4 -form $\Omega_{\mathrm{WZ}}$ itself. Unlike the 4-form, the 5-form is manifestly chirally symmetric, and it's also a much simpler function of the non-linear scalar field $\Omega_{\ell}$. The action (3.4) can be written in terms of the 5 -form integrated over 5 dimensions: the ordinary four, plus an auxiliary fifth dimension. But that fifth dimension has absolutely nothing to do with the deconstructed fifth dimension of the $\mathrm{SQCD}_{5}$, so to avoid dimensional confusion, we use the $4 \mathrm{D}$ form of the Wess-Zumino action in this paper. 
is the Chern-Simons 5-form. The coefficient $k_{\mathrm{cs}}$ of the Chern-Simons coupling (3.6) is quantized; in light of the above,

$$
k_{\mathrm{cS}}=k_{\mathrm{wz}}=n_{c}-n_{f} .
$$

Note that the effective low-energy theory in the 5D continuum limit is a pure SYM whereas all the quarks which were present in the 4D theory have decoupled at the deconstruction threshold $E \sim(1 / a)$. Nevertheless, thanks to those decoupled quarks, the Chern-Simons level of the SYM theory is lowered from $k_{\mathrm{cs}}=n_{c}$ for the quark-less quiver we have studied in $[20]$ down to $k_{\mathrm{cs}}=n_{c}-n_{f}$.

The general case which allows $\mu_{f} \neq 0$ is more complicated: The quark masses relate fermions $\Psi_{\ell}^{Q}$ and $\Psi_{\ell}^{\widetilde{Q}}$ which couple to different sigma models and we no longer have isolated sigma models with separate Wess-Zumino couplings. Hence, instead of a direct deconstruction of the 5D Chern-Simons coupling (3.7), we assume it exists at some level $k_{\mathrm{cs}}$ and calculate this level by taking the very-low-energy limit $E \ll(1 / L a)$. In this limit, the $5 \mathrm{D}$ theory is dimensionally reduced to 4D (without the Kaluza-Klein excitations), the $A^{4}$ component of the vector field becomes a scalar, and the CS coupling becomes a field-dependent set of $\Theta$ angles:

$$
\mathcal{L}_{\mathrm{cs}}^{4 \mathrm{D}}=\oint_{x^{4}} k_{\mathrm{cs}} \Omega_{\mathrm{CS}}\left(A_{5 \mathrm{D}}^{\mu}\right)=\frac{i k_{\mathrm{cs}} L a}{8 \pi^{2}} \operatorname{Tr}\left(A^{4} F \wedge F\right)=\frac{i}{16 \pi^{2}} \sum_{i}\left(k_{\mathrm{cs}} L a A_{i}^{4}\right) F_{i} \wedge F_{i}
$$

where in the last equality here we have restricted the $4 \mathrm{D}$ gauge fields to the abelian $F_{i}$ $\left(i=1,2, \ldots, n_{c}, \sum_{i} F_{i}=0\right)$ which remain massless after the Wilson lines $L a A_{4}^{i}$ break the $S U\left(n_{c}\right)$ down to the $U(1)^{n_{c}-1}$.

From the quiver point of view, the

$$
\Theta_{i}=k_{\mathrm{cs}} \times \operatorname{La} A_{i}^{4}
$$

are $\Theta$ angles which arise from the field-dependent masses of the charged fermions. The 
Adler-Bardeen theorem provides an exact formula:

$$
\Theta_{i}=-\sum_{q} q_{i}^{2} \arg \operatorname{det}\left(M_{q}\right)
$$

where $q$ is the array of abelian charges $\left(q_{1}, q_{2}, \ldots, q_{n_{c}}\right)$ and $M_{q}$ is the mass matrix of fermions with the same charges $q$. Our task therefore is to evaluate this formula and show that the $\Theta_{i}$ angles indeed have form (3.10) for the Chern-Simons level $k_{\mathrm{cs}}$ specified in eq. (3.1).

In the eigen-basis of the Wilson lines, the fermionic mass matrix pairs the quarks $\left(\Psi_{\ell}^{Q}\right)^{i, f}$ with the antiquarks $\left(\Psi_{\ell^{\prime}}^{\widetilde{Q}}\right)^{i, f}$ and the gauginos $\left(\lambda_{\ell}\right)^{i j}$ with the link fermions $\left(\Psi_{\ell^{\prime}}^{\Omega j i}\right.$ :

$$
\begin{aligned}
\mathcal{L}_{\text {mass }}^{\text {fermion }}= & \sum_{i, f} \sum_{\ell, \ell^{\prime}} M_{\ell, \ell^{\prime}}\left[Q^{i, f}\right] \times\left(\Psi_{\ell}^{Q}\right)^{i, f}\left(\Psi_{\ell^{\prime}}^{\widetilde{Q}}\right)^{i, f} \\
& +\sum_{i, j} \sum_{\ell, \ell^{\prime}} M_{\ell, \ell^{\prime}}\left[\lambda^{i j}\right] \times\left(\lambda_{\ell}\right)^{i j}\left(\Psi_{\ell^{\prime}}^{\Omega}\right)^{j i}, \\
\text { where } M_{\ell, \ell^{\prime}}\left[Q^{i, f}\right]= & \gamma v e^{a \varphi_{i}} \times \delta_{\ell+1, \ell^{\prime}}-\gamma \mu_{f} \times \delta_{\ell, \ell^{\prime}} \\
\text { and } M_{\ell, \ell^{\prime}}\left[\lambda^{i j}\right]= & \left(g v e^{a \varphi_{i}}\right)^{*} \times \delta_{\ell, \ell^{\prime}+1}-\left(g v e^{a \varphi_{j}}\right)^{*} \times \delta_{\ell, \ell^{\prime}} .
\end{aligned}
$$

The determinants of the mass matrices with respect to the quiver indices $\ell, \ell^{\prime}$ are completely straightforward:

$$
\begin{aligned}
\operatorname{det} M\left[Q^{i, f}\right] & = \pm \gamma^{L}\left(v^{L} \exp \left(L a \varphi_{i}\right)-\mu_{f}^{L}\right), \\
\operatorname{det} M\left[\lambda_{j}^{i}\right] & =\left(g v^{*}\right)^{L}\left(\exp \left(L a \varphi_{i}^{*}\right)-\exp \left(L a \varphi_{j}^{*}\right)\right) .
\end{aligned}
$$

Taking into account the abelian charges of the gauginos, their combined contribution to the $\Theta_{i}$ angle (3.11) amounts to

$$
\left[\Theta_{i}\right]_{\lambda}=-\sum_{j \neq i} \arg \left(\operatorname{det} M\left[\lambda^{i j}\right] \times \operatorname{det} M\left[\lambda^{j i}\right]\right)
$$

Assuming for simplicity that the 5D scalar $\phi_{i}$ have zero VEVs and only the Wilson lines break the $S U\left(n_{c}\right)$ - thus $\varphi_{j}=i A_{j}^{4}$ - we have

$$
\operatorname{det} M\left[\lambda^{i j}\right] \times \operatorname{det} M\left[\lambda^{j i}\right]=4\left(g v^{*}\right)^{2 L} \sin ^{2} \frac{L a}{2}\left(A_{i}^{4}-A_{j}^{4}\right) \times \exp \left(-i L a\left(A_{i}^{4}+A_{j}^{4}\right)\right),
$$


and therefore

$$
\left[\Theta_{i}\right]_{\lambda}=\sum_{j \neq i}\left(L a\left(A_{i}^{4}+A_{j}^{4}\right)+2 L \arg (v)\right)=n_{c} \times L a A_{i}^{4}+\text { const. }
$$

Now consider the quark mass determinant (3.15). In the large $L$ limit,

$$
\operatorname{det} M\left[Q^{i, f}\right] \approx \gamma^{L} \begin{cases} \pm v^{L} \exp \left(i L a A_{i}^{4}\right) & \text { when }|v|>\left|\mu_{f}\right| \\ \mp \mu_{f}^{L} & \text { when }|v|<\left|\mu_{f}\right|\end{cases}
$$

hence the quark contribution to the $\Theta_{i}$ angle (3.11)

$$
\begin{aligned}
{\left[\Theta_{i}\right]_{Q} } & =-\sum_{f}^{\left|\mu_{f}\right|<|v|}\left(L a A_{i}^{4}+L \arg (\gamma v)\right)-\sum_{f}^{\left|\mu_{f}\right|>|v|} L \arg \left(\gamma \mu_{f}\right) \\
& =\text { const }-\left(L a A_{i}^{4}\right) \times \#\left\{f:\left|\mu_{f}\right|<|v|\right\} .
\end{aligned}
$$

Totaling the quark and the gaugino contributions, we arrive at

$$
\Theta_{i}=k_{\mathrm{cs}}^{\prime} \times\left(L a A_{i}^{4}\right)+\text { const }
$$

in full agreement with the dimensionally reduced Chern-Simons coupling (3.10) for

$$
k_{\mathrm{cs}}^{\prime}=n_{c}-\#\left\{f:\left|\mu_{f}\right|<|v|\right\} .
$$

Although the above Chern-Simons level $k_{\mathrm{cs}}^{\prime}$ is not quite as in eq. (3.1), the discrepancy involves only quarks with $4 \mathrm{D}$ masses $\mu_{f} \approx v$. The problem lies in our taking the lowenergy limit too literally and hence integrating out any $4 \mathrm{D}$ fermionic mode which is not exactly massless, including all of the quark modes. Consequently, the resulting ChernSimons level $k_{\mathrm{cs}}^{\prime}$ corresponds to the low-energy limit of the $\mathrm{SQCD}_{5}$ from which all the quarks have been integrated out. Thus,

$$
k_{\mathrm{cs}}^{\prime}=k_{\mathrm{cs}}+\sum_{f} \frac{1}{2} \operatorname{sign}\left(\operatorname{Re} m_{f}\right)
$$

where $k_{\mathrm{cs}}$ refers to the 5D theory which has light quarks only (the 5D masses $\left|m_{f}\right| \leq m_{\max } \ll$ $(1 / a))$, and only such light quarks appear in the $\sum_{f}$. In terms of the $4 \mathrm{D}$ masses $\mu_{f}=v e^{a m_{f}}$ 
(cf. eq. (2.29)), this means

$$
k_{\mathrm{cs}}^{\prime}=k_{\mathrm{cs}}+\frac{1}{2} \#\left\{f:|v|<\left|\mu_{f}\right|<|v|+m_{\max }\right\}-\frac{1}{2} \#\left\{f:|v|-m_{\max }<\left|\mu_{f}\right|<|v|\right\} .
$$

Finally, comparing this formula to eq. (3.23) we arrive at

$$
k_{\mathrm{cs}}=n_{c}-\#\left\{f:\left|\mu_{f}\right|<|v|-m_{\max }\right\}-\frac{1}{2} \#\left\{f:|v|-m_{\max }<\left|\mu_{f}\right|<|v|+m_{\max }\right\}
$$

which is exactly what we have promised in eq. (3.1) (but now have restated in a more precise manner).

Eq. (3.26) tells us that deconstructing $\mathrm{SQCD}_{5}$ with a given Chern-Simons level may require more quark flavors in the $4 \mathrm{D}$ quiver theory then are present in 5D. To avoid notational confusion, let $n_{f}$ henceforth refer to the number of 5D quark flavors with masses $|m| \ll(1 / a)$ while $F$ denotes the total number of $4 \mathrm{D}$ flavors of the quiver. According to eq. (3.26), we need

$$
F=n_{f}+\Delta F \quad \text { where } \Delta F=n_{c}-\frac{n_{f}}{2}-k_{\mathrm{cs}} ;
$$

for $f=1,2, \cdots, n_{f}$ the $4 \mathrm{D}$ quark masses should be set to $\mu_{f}=v e^{a m_{f}}$ according to eq. (2.29), but for $f>n_{f}$ we want $\mu_{f} \ll v$; for simplicity, we let $\mu_{f}=0$ for $f=\left(n_{f}+1\right), \ldots, F$.

Note that quantum consistency of $\mathrm{SQCD}_{5}$ requires integer $k_{\mathrm{cs}}$ when $n_{f}$ is even but halfinteger $k_{\mathrm{cs}}$ when $n_{f}$ is odd. Also, positivity of the moduli-dependent gauge couplings (1.2-5) for $h>0$ requires

$$
\left|k_{\mathrm{cS}}\right|+\frac{n_{f}}{2} \leq n_{c}
$$

In terms of eq. (3.27), these rules translate to $\Delta F$ being a non-negative integer (good, since otherwise deconstruction would be impossible) and $F \leq 2 n_{c}$. Since each $S U\left(n_{c}\right)_{\ell}$ gauge group of the quiver couples to the total of $\left(n_{c}+F\right)$ chiral fields in the $(\square+\bar{\square})_{\ell}$ representation, this means that the quiver theory should be asymptotically free, or at least asymptotically finite. This is good for the quiver as a UV completion of the 5D theory, but it also means strong quantum corrections in the IR limit of the quiver theory. In $\S 6$, we shall see that when such quantum corrections become strong enough, the deconstructed $\mathrm{SQCD}_{5}$ may have a flop transition to a different 5D phase. 
We conclude this section with a few words about $\mathrm{SQCD}_{5}$ theories with only two colors [2]. The $S U(2)$ group does not have a cubic invariant, hence the 5D Chern-Simons coupling does not exist for $n_{c}=2$. Instead, there is a $\mathbb{Z}_{2}$ topological invariant and hence a vacuum angle $\theta$ which takes 2 discrete values 0 and $\pi$ (modulo $2 \pi$ ). It would be interesting to deconstruct this vacuum angle directly from the quantum quiver theory, but here we prefer a simpler derivation: Let us realize an $S U(2)$ quiver theory with $F 4 \mathrm{D}$ flavors as the mesonic Higgs branch of an $S U(3)$ quiver with $F^{\prime}=F+2$. This gives us two ways to deconstruct the 5D theory with $n_{c}=2$ : We may first deconstruct an $S U(3)$ theory with $n_{f}^{\prime}=n_{f}+2$, then Higgs the $5 \mathrm{D}$ theory down to $S U(2)$ (which eats up the two extra flavors), hence

$$
[\theta]_{S U(2)}=\pi \times\left[k_{\mathrm{cs}}\right]_{S U(3)}=\pi \times\left[n_{c}^{\prime}-\frac{1}{2} n_{f}^{\prime}-\Delta F\right]_{S U(3)}=\pi \times\left[n_{c}-\frac{1}{2} n_{f}-\Delta F\right]_{S U(2)}
$$

Alternatively, we may first Higgs the $S U(3)$ down to $S U(2)$ in $4 \mathrm{D}$ and deconstruct afterward, but the end result should be the same, thus

$$
\text { for } n_{c}=2, \quad \theta=\pi \times \Delta F-\frac{\pi}{2} \times n_{f} \text { modulo } 2 \pi \text {. }
$$

Note that for odd $n_{f}$ this angle takes values $\pm \pi / 2$ instead of 0 or $\pi$, but this is OK since the real vacuum angle obtains only after after integration out of the 5D fermions, thus

$$
\bar{\theta}=\theta+\frac{\pi}{2} \sum_{f} \operatorname{sign}\left(\operatorname{Re} m_{f}\right)=\pi \times\left(\Delta F+\#\left\{f: m_{f}<0\right\}\right)
$$

which indeed takes values 0 and $\pi$ for any $n_{f}$.

Another peculiarity of the $\mathrm{SQCD}_{5}$ with $n_{c}=2$ is that it allows up to 7 quark flavors instead of usual limit $n_{f} \leq 2 n_{c}$ for $n_{c} \geq 3$. The $S U(2)$ quiver theory however loses asymptotic freedom and becomes UV-divergent and IR-trivial for $n_{f}>4$. Consequently, at the quantum level, the quiver (2.1) fails to deconstruct $\mathrm{SQCD}_{5}$ with $n_{c}=2$ and $n_{f}=5,6$ or 7 . We suspect such theories can be deconstructed in terms of more complicated quivers and we hope to present them in a future publication, but in this article we shall henceforth assume $F \leq 2 n_{c}$ even for $n_{c}=2$. 


\section{Quantum Deconstruction of the Gauge Couplings}

The deconstructed $\mathrm{SQCD}_{5}$ has only 4 exact supersymmetries, hence at the non-perturbative level of analysis only the holomorphic features of the 4D quiver are exactly calculable. From the deconstruction point of view, the most important holomorphic feature is the moduli dependence of the abelian gauge couplings $\tau_{i j}(\varphi)$ for the Coulomb branch of the quiver's moduli space. Whereas the unbroken gauge symmetry $(U(1))^{n_{c}-1} \subset S U\left(n_{c}\right)_{\text {diag }}$ of the quiver deconstructs the Kaluza-Klein reduction of unbroken 5D symmetry on a circle of length $2 \pi R=L a$, in the large quiver limit $L \rightarrow \infty$ we should have

$$
2 \pi \operatorname{Im} \tau_{i j}(\varphi) \equiv\left[\frac{8 \pi^{2}}{g_{4}^{2}(\varphi)}\right]_{i j}=L a \times\left[\frac{8 \pi^{2}}{g_{5}^{2}(\phi)}\right]_{i j}+O(1) .
$$

In this section, we shall see that this is indeed the case, and furthermore the $5 \mathrm{D}$ gauge couplings on the right hand side of eq. (4.1) are exactly as in eqs. (1.2-5) for the un-deconstructed 5 D SQCD.

In a separate article [25] we have analyzed the entire chiral ring of the $4 \mathrm{D}$ quiver theory (2.1); for the present purposes, let us simply state without proof the key results which are relevant for the gauge couplings. First of all, the Seiberg-Witten spectral curve of the quiver is the Riemann surface of the quadratic equation

$$
Y^{2}-Y \times P(X)+(-1)^{F} \alpha B(X)=0
$$

where $P(X)$ and $B(X)$ are polynomials of respective degrees $n_{c}$ and $F$, and $\alpha$ is a constant parametrizing the non-perturbative quantum effects. Specifically, $\alpha$ originates at the diagonal instanton level of the $\left[S U\left(n_{c}\right)\right]^{L}$ quiver, meaning one instanton of the diagonal $S U\left(n_{c}\right)_{\text {diag }}$ gauge group, or equivalently, one instanton in each of the $S U\left(n_{c}\right)_{\ell}$ factor. For $F<2 n_{c}$,

$$
\alpha=\left((-\gamma)^{F} \Lambda^{2 n_{c}-F}\right)^{L}
$$

where $\Lambda$ is the usual dimensional transmutant of the asymptotically free $4 \mathrm{D}$ gauge coupling 
$g$ (note same $g_{\ell} \equiv g$ for all $\ell$ hence same $\Lambda_{\ell} \equiv \Lambda$ ). In the asymptotically-flat case of $F=2 n_{c}$

$$
\alpha \approx\left[\gamma^{2 n_{c}} \exp \left(2 \pi i \tau_{\mathrm{UV}}\right)\right]^{L} \approx\left[\exp \left(i \theta-\frac{8 \pi^{2}}{g_{\mathrm{phys}}^{2}}\right)\right]^{L}
$$

As to the polynomials,

$$
B(X)=\prod_{f=1}^{F}\left(X-\mu_{f}\right)=X^{\Delta F} \times \prod_{f=1}^{n_{f}}\left(X-\mu_{f}\right)
$$

parametrizes the bare 4D quark masses $\mu_{f}$ of the theory, and

$$
P(X)=\prod_{i=1}^{n_{c}}\left(X-\varpi_{i}\right)
$$

parametrizes the Coulomb moduli space of the quiver. Since this space has only $n_{c}-1$ independent moduli, the roots $\varpi_{i}$ of $P(X)$ are subject to one constraint, namely

$$
\prod_{i=1}^{n_{c}} \varpi_{i} \equiv V^{L n_{c}}=\text { const }
$$

〈4) The second equality here follows from eq. (2.19) for the renormalized gauge and Yukawa couplings, and the approximation is ignoring the threshold effects. Instead, we use the massless renormalization group equations for the $g_{\text {ren }}(E)$ and $\gamma_{\text {ren }}(E)$ and then impose $g_{\text {ren }}(E)=\gamma_{\text {ren }}(E)$ for some low-energy normalization point $E$. In terms of the running kinetic energy factors $Z_{\Omega}(E), Z_{Q}(E)$ and $Z_{\widetilde{Q}}(E)$ for the charged fields, the renormalized Yukawa and gauge couplings are given by

and

$$
\gamma_{\text {ren }}^{2}(E)=\frac{\left|\gamma_{\text {holomorphic }}\right|^{2}}{Z_{\Omega}(E) Z_{Q}(E) Z_{\widetilde{Q}}(E)}
$$

$$
g_{\mathrm{ren}}^{2 n_{c}}(E) \times \exp \left(\frac{8 \pi^{2}}{g_{\mathrm{ren}}^{2}(E)}\right)=\frac{\exp \left(2 \pi \operatorname{Im} \tau_{\mathrm{UV}}\right) \times(E / \text { cutoff })^{2 n_{c}-F}}{\left(Z_{\Omega}(E)\right)^{n_{c}}\left(Z_{Q}(E) Z_{\widetilde{Q}}(E)\right)^{F}} .
$$

Substituting $F=2 n_{c}$ and combining the two equations, we obtain

$$
\left(\frac{\gamma_{\text {ren }}}{g_{\text {ren }}}\right)^{2 n_{c}} \times \exp \left(-\frac{8 \pi^{2}}{g_{\text {ren }}^{2}}\right) \equiv\left|\gamma_{\text {hol }}^{2 n_{c}} \exp \left(2 \pi \tau_{\mathrm{UV}}\right)\right|
$$

at all renormalization scales. Eq. (4.4) follows from this formula once we identify $g_{\mathrm{phys}}=g_{\mathrm{ren}}(E)$ and $\gamma_{\mathrm{phys}}=\gamma_{\mathrm{ren}}(E)$ for some renormalization point $E$ and apply eq. (2.19). 
where

$$
\begin{aligned}
V^{L n_{c}} & =\left(v_{1}^{n_{c}}\right)^{L}+\left(v_{2}^{n_{c}}\right)^{L} \text { for } \\
v_{1}^{n_{c}}+v_{2}^{n_{c}} & =v^{n_{c}} \text { and } v_{1}^{n_{c}} \times v_{2}^{n_{c}}=\Lambda^{2 n_{c}-F}\left(\gamma \mu_{1}\right)\left(\gamma \mu_{2}\right) \cdots\left(\gamma \mu_{F}\right) .
\end{aligned}
$$

Qualitatively,

$$
\begin{aligned}
V & =v, \text { exactly, } & \text { for } \Delta F>0, \\
\text { but } V & =v+O\left(\Lambda^{2 n_{c}-F}\right) & \text { for } \Delta F=0 .
\end{aligned}
$$

The gauge coupling matrix $\tau_{i j}$ follows directly from the spectral curve (4.2), but in order to study its dependence on the deconstruction-appropriate moduli $\varphi_{j}=\phi_{j}+i A_{j}^{4}$ we must first define the $\varphi_{j}$ in a gauge-invariant way. Classically, there is a simple definition in terms of eigenvalues of the quiver-ordered product $\Omega_{L} \Omega_{L-1} \cdots \Omega_{2} \Omega_{1}$ of the bi-fundamental link fields:

$$
\text { eigenvalues }\left[\Omega_{L} \Omega_{L-1} \cdots \Omega_{2} \Omega_{1}\right]=\left(\left[v e^{a \varphi_{1}}\right]^{L},\left[v e^{a \varphi_{2}}\right]^{L}, \ldots,\left[v e^{a \varphi_{n_{c}}}\right]^{L}\right) \text {, }
$$

cf. eq. (2.23). Or equivalently, we may define the resolvent function and look for its poles:

$$
T(X) \stackrel{\text { def }}{=} \operatorname{Tr} \frac{1}{X-\Omega_{L} \cdots \Omega_{1}} \stackrel{\text { cla }}{=} \sum_{i=1}^{n_{c}} \frac{1}{X-\left[v \exp \left(a \varphi_{i}\right)\right]^{L}}
$$

Unfortunately, in the quantum theory the resolvent is defined as

$$
T(X)=\left\langle\operatorname{Tr} \frac{1}{X-\Omega_{L} \cdots \Omega_{1}}\right\rangle \quad \text { instead of } \operatorname{Tr} \frac{1}{X-\left\langle\Omega_{L}\right\rangle \cdots\left\langle\Omega_{1}\right\rangle}
$$

and consequently it has branch cuts instead of poles; specifically,

$$
T(X)=\frac{\partial_{X} Y}{Y}=\frac{1}{\sqrt{P^{2}-4(-1)^{F} \alpha B}}\left(\partial_{X} P-\frac{2(-1)^{F} \alpha}{P+\sqrt{P^{2}-4(-1)^{F} \alpha B}} \partial_{X} B\right)
$$

However, in the weak coupling limit $\Lambda \rightarrow 0$, the branch cuts become very short and can be 
approximated as poles located at the roots $\varpi_{i}$ of the $P(X)$ polynomial:

$$
\Lambda \rightarrow 0 \Longrightarrow \alpha B \ll P^{2} \Longrightarrow T(X) \approx \frac{\partial_{X} P}{P}=\sum_{i=1}^{n_{c}} \frac{1}{X-\varpi_{i}}
$$

which immediately suggests the definition

$$
\varphi_{i} \stackrel{\text { def }}{=} \frac{1}{a} \log \frac{\sqrt[L]{\varpi_{i}}}{v} \Longleftrightarrow P(X)=\prod_{i=1}^{n_{c}}\left(X-v^{L} \exp \left(L a \varphi_{i}\right)\right) .
$$

Or rather

$$
\varphi_{i} \stackrel{\text { def }}{=} \frac{1}{a} \log \frac{\sqrt[L]{\varpi_{i}}}{V} \Longleftrightarrow P(X)=\prod_{i=1}^{n_{c}}\left(X-V^{L} \exp \left(\operatorname{La} \varphi_{i}\right)\right)
$$

in order to map the quantum-corrected moduli constraint (4.7) onto classical-like trace condition $\sum_{i} \varphi_{i}=0$.

Outside the week coupling limit, $T(X)$ generally has branch cuts of finite length; however, at a point where the Coulomb branch of the quiver's moduli space joins a mesonic Higgs branch, one of the branch cuts does degenerate into a pole. In terms of the Seiberg-Witten spectral curve, this happens when a root of $P(X)$ coincides with a double root of $B(X)$, eg. $\varpi_{1}=\mu_{1}^{L}=\mu_{2}^{L}$ : At this point, the quadratic equation (4.2) factorizes as

$$
Y=\left(X-\varpi_{1}\right) \times \tilde{Y}, \quad \widetilde{Y}^{2}-\tilde{Y} \times \frac{P(X)}{\left(X-\varpi_{1}\right)}+\frac{(-1)^{F} \alpha B(X)}{\left(X-\mu_{1,2}^{L}\right)^{2}}=0,
$$

where the second eq. (4.18) describes a hyperelliptic curve of reduced genus ( $g=n_{c}-2$ instead of $\left.g=n_{c}-1\right)$ - which corresponds to the reduced rank of the Higgs branch's gauge symmetry $(U(1))^{n_{c}-2}$ - and the resolvent

$$
T(X)=\frac{\partial_{X} Y}{Y}=\frac{1}{X-\varpi_{1}}+\frac{\partial_{X} \tilde{Y}}{\widetilde{Y}}
$$

has a pole at $X=\varpi_{1}$ on both sheets of the Riemann surface.

From the 5D point of view, this point of the moduli space corresponds to $\varphi_{1}=m_{1}=m_{2}$. Therefore, the $4 \mathrm{D} \leftrightarrow 5 \mathrm{D}$ map of moduli and masses should have $\varphi_{i}=m_{f}$ exactly when $\varpi_{i}=\mu_{f}^{L}$, regardless of any quantum corrections. Hence, the classical mass formula (2.29) 
calls for defining the 5D Coulomb moduli according to eq. (4.16), weak coupling or strong coupling. Alternatively, we may rescale $v \rightarrow V$ in both mass and moduli maps; this gives us eq. (4.17) for the moduli - and hence $\sum_{i} \varphi_{i}=0$ - while the 5D quark masses are given by

$$
m_{f} \stackrel{\text { def }}{=} \frac{1}{a} \log \frac{\mu_{f}}{V}
$$

In light of the definitions (4.17) and (4.20), it is convenient to rescale the $X$ and $Y$ coordinates of the spectral curve by appropriate powers of $V$ and rewrite the curve as

$$
y^{2}-y \times p(x)+e^{-L a S} b(x)=0
$$

where

$$
\begin{aligned}
p(x) & =\prod_{i=1}^{n_{c}}\left(x-e^{L a \varphi_{i}}\right), \\
b(x) & =(-1)^{F} x^{\Delta F} \times \prod_{i=1}^{n_{f}}\left(x-e^{L a m_{f}}\right), \\
\text { and } e^{-L a S} & =\frac{\alpha}{V^{L\left(2 n_{c}-F\right)}} \\
& \Uparrow \\
S & \stackrel{\text { def }}{=} \frac{1}{a} \times \begin{cases}\log \frac{V^{2 n_{c}-F}}{(-\gamma)^{F} \Lambda^{2 n_{c}-F}} & \text { for } F<2 n_{c}, \\
\frac{8 \pi^{2}}{g^{2}}-i \theta & \text { for } F=2 n_{c} .\end{cases}
\end{aligned}
$$

In these notations, eqs. (4.21-23) describe the spectral curve of a 5D theory compactified on a circle of length $2 \pi R=L a$ without any indication that the compactified dimension is discrete; all the $4 \mathrm{D}$ aspects of the deconstructed $\mathrm{SQCD}_{5}$ are 'hiding' in the definitions (4.17), (4.20) and (4.24) of the 5D moduli and parameters. In other words, the details of the deconstruction decouple from the spectral curve (4.21-23) of the compactified $\mathrm{SQCD}_{5}$.

Thanks to this decoupling, the spectral curve has all the symmetries of the $5 \mathrm{D}$ theory, even when the 4D quiver theory does not respect them at the non-holomorphic level. From the deconstruction point of view, such enhanced symmetries of the $4 \mathrm{D}$ spectral curve acts as custodial symmetries of the 5D symmetries. For example, consider the $\mathbf{C}$ symmetry which 
acts on spectral curves according to

$$
\begin{gathered}
x \rightarrow \frac{1}{x}, \quad y \rightarrow \frac{y}{(-x)^{n_{c}}}, \\
\Delta F \rightarrow 2 n_{c}-n_{f}-\Delta F, \quad \varphi_{j} \rightarrow-\varphi_{j}, \quad m_{f} \rightarrow-m_{f}, \\
S \rightarrow S-\sum_{f=1}^{n_{f}} m_{f},
\end{gathered}
$$

or equivalently

$$
H \stackrel{\text { def }}{=} S-\frac{1}{2} \sum_{f=1}^{n_{f}} m_{f} \quad \text { is invariant. }
$$

Note that $\mathbf{C}$ is not a symmetry of the quiver theories themselves but only of their spectral curves, or more accurately, it's a symmetry of the family of such spectral curves with variable $m_{f}$ and $\Delta F$ parameters (but fixed $n_{c}$ and $n_{f}$ ). Nevertheless, the very existence of this $4 \mathrm{D}$ symmetry implies a 5D symmetry which acts according to eq. (4.26) translated into 5D terms, namely

$$
k_{\mathrm{cs}} \rightarrow-k_{\mathrm{cs}}, \quad \phi_{j} \rightarrow-\phi_{j}, \quad A_{j}^{\mu} \rightarrow-A_{j}^{\mu}, \quad m_{f} \rightarrow-m_{f} .
$$

In other words, $\mathbf{C}$ acts as a custodial symmetry of the 5D charge conjugation symmetry.

After all these preliminaries, let us consider the decompactification limit $L a \rightarrow \infty$ of the spectral curve (4.21-23). In this limit, the moduli $\varphi_{i}$ and the parameters $S$ and $m_{f}$ remain fixed, hence the $x$ and $y$ coordinates of the curve scale as

$$
x=\exp (L a \times \xi), \quad y=\exp (L a \times \eta)
$$

for fixed $\xi$ and $\eta$. Hence,

$$
\begin{aligned}
& p(x) \sim \exp (L a \times O(\xi, \varphi)) \quad \text { except for } \xi \text { very near one of the } \varphi_{i} \\
& b(x) \sim \exp (L a \times O(\xi, m)) \quad \text { except for } \xi \text { very near one of the } m_{f}
\end{aligned}
$$


and the ratio

$$
\frac{e^{-L a S} b(x)}{p^{2}(x)} \sim \exp [-L a \times(S+O(\xi, \varphi, m))]
$$

generally becomes (for $L a \rightarrow \infty$ ) either extremely large or extremely small, depending on $S$. For sufficiently large $S$ (i.e., for sufficiently weakly coupled quiver theory, cf. eq. (4.24)), the exponent on the right hand side of eq. (4.32) is generally negative, and therefore

$$
e^{-L a S} b(x) \ll p^{2}(x) \quad \text { except for } x \text { very near one of the } e^{L a \varphi_{i}} \text {. }
$$

Consequently, the roots $x_{1}, x_{2}, \ldots, x_{2 n_{c}}$ of the discriminant

$$
D(x)=p^{2}(x)-4 e^{-L a S} b(x)
$$

cluster in tight pairs near the roots $e^{L a \varphi_{i}}$ of $p(x)$. Assuming for simplicity a generic point of the Coulomb moduli space where all the $\varphi_{i}$ are distinct, we have

$$
x_{2 i-1}, x_{2 i} \approx e^{\operatorname{La\varphi _{i}}} \pm d_{i} \quad\left(i=1, \ldots, n_{c}\right)
$$

where

$$
d_{i}=2 e^{-L a S / 2} \times \frac{\sqrt{b\left(e^{L a \varphi_{i}}\right)}}{p^{\prime}\left(e^{L a \varphi_{i}}\right)} \ll\left(e^{L a \varphi_{j}}-e^{L a \varphi_{i}}\right) \forall j \neq i .
$$

From the spectral curve's point of view, the discriminant roots $x_{1}, \ldots, x_{2 n_{c}}$ are branching points of the Riemann surface (4.21) over the $X$ plane, and their clustering into tight pairs means that in a suitable basis of the abelian gauge fields of the theory, all gauge couplings are weak. Specifically, the abelian coupling matrix is given [20] by

$$
\left.\begin{array}{rl}
\tau_{i \neq j} & =\frac{i}{2 \pi} \log \frac{e^{L a \varphi_{i}} e^{L a \varphi_{j}}}{\left(e^{L a \varphi_{i}}-e^{L a \varphi_{j}}\right)^{2}} \\
\tau_{i=j} & =\frac{i}{2 \pi} \log \frac{\left(e^{L a \varphi_{i}}\right)^{2}}{\left(d_{i} / 2\right)^{2}}
\end{array}\right\} \text { modulo } \mathbb{Z}
$$

and all we need to do now is to calculate the $d_{i}$ according to eq. (4.36).

To avoid compactification artefacts due to finite size $L a$ of the deconstructed dimension, we strengthen the assumption of the $\varphi_{i}$ being all distinct and assume that the differences 
are larger than the compactification scale:

$$
\forall i \neq j:\left|\operatorname{Re}\left(\varphi_{i}-\varphi_{j}\right)\right| \gg \frac{1}{L a}
$$

hence $\forall i \neq j$ either $e^{L a \varphi_{i}} \gg e^{L a \varphi_{j}}$ or vice verse $e^{L a \varphi_{i}} \ll e^{L a \varphi_{j}}$. This allows us to approximate

$$
\begin{aligned}
\left(e^{L a \varphi_{i}}-e^{L a \varphi_{j}}\right) & \approx \max \left(+e^{L a \varphi_{i}},-e^{L a \varphi_{j}}\right) \\
& = \pm \exp \left(\frac{L a}{2}\left(\varphi_{i}+\varphi_{j}+\left\lfloor\varphi_{i}-\varphi_{j}\right\rfloor\right)\right)
\end{aligned}
$$

where $\left\lfloor\varphi_{i}-\varphi_{j}\right\rfloor$ denotes $\left(\varphi_{i}-\varphi_{j}\right) \times \operatorname{sign} \operatorname{Re}\left(\varphi_{i}-\varphi_{j}\right){ }^{\langle 5\rangle}$ Likewise, we assume no coincidences between the moduli $\varphi_{i}$ and the masses $m_{f}$, hence

$$
\left(e^{L a \varphi_{i}}-e^{\operatorname{Lam}_{f}}\right) \approx \pm \exp \left(\frac{L a}{2}\left(\varphi_{i}+m_{f}+\left\lfloor\varphi_{i}-m_{f}\right\rfloor\right)\right)
$$

Thanks to approximations (4.39) and (4.40), we have

$$
\begin{aligned}
\tau_{i \neq j}(\varphi) & \approx \frac{-i L a}{2 \pi} \times\left\lfloor\varphi_{i}-\varphi_{j}\right\rfloor \\
e^{L a \varphi_{i}} \times p^{\prime}\left(e^{L a \varphi_{i}}\right) & \approx \pm \exp \left(\frac{L a}{2}\left(n_{c} \varphi_{i}+\sum_{k}\left\lfloor\varphi_{i}-\varphi_{k}\right\rfloor\right)\right) \\
b\left(e^{L a \varphi_{i}}\right) & \approx \pm \exp \left(\frac{L a}{2}\left(\left(2 \Delta F+n_{f}\right) \varphi_{i}+\sum_{f} m_{f}+\sum_{f}\left\lfloor\varphi_{i}-m_{f}\right\rfloor\right)\right),
\end{aligned}
$$

hence

$$
\begin{aligned}
\tau_{i=j}(\varphi) & \approx \frac{i}{2 \pi} \log \frac{\left(e^{\operatorname{La\varphi _{i}}} p^{\prime}\left(e^{\operatorname{La\varphi _{i}}}\right)\right)^{2}}{e^{-\operatorname{LaS}} b\left(e^{\left.\operatorname{La\varphi _{i}}\right)}\right.} \\
& \approx \frac{i L a}{2 \pi} \times\left(\begin{array}{c}
H+\left(n_{c}-\Delta F-\frac{n_{f}}{2}\right) \times \varphi_{i} \\
+\sum_{k}\left\lfloor\varphi_{i}-\varphi_{k}\right\rfloor-\frac{1}{2} \sum_{f}\left\lfloor\varphi_{i}-m_{f}\right\rfloor
\end{array}\right) \text { modulo } \frac{\mathbb{Z}}{2},
\end{aligned}
$$

where

$\langle 5\rangle$ For a real number $\alpha,\lfloor\alpha\rfloor \equiv|\alpha|$, but for complex numbers we need a new notation. However, for any complex number $\beta, \operatorname{Re}(\lfloor\beta\rfloor)=|\operatorname{Re}(\beta)|$. 


$$
H \stackrel{\text { def }}{=} S-\frac{1}{2} \sum_{f=1}^{n_{f}} m_{f}
$$

and all approximations become exponentially good in the large quiver limit $L \rightarrow \infty$.

According to eqs. (4.41) and (4.44), the entire abelian coupling matrix $\tau_{i j}(\varphi)$ of the quiver is proportional to La, exactly as promised in eq. (4.1). Furthermore, the deconstructed 5D gauge couplings of the $\mathrm{SQCD}_{5}$ 's Coulomb branch

$$
\left[\frac{8 \pi^{2}}{g_{5}^{2}}\right]_{i \neq j}=-\left|\phi_{i}-\phi_{j}\right|
$$

and

$$
\left[\frac{8 \pi^{2}}{g_{5}^{2}}\right]_{i=j}=\operatorname{Re}(H)+k_{\mathrm{cs}} \times \phi_{i}+\sum_{k}\left|\phi_{i}-\phi_{k}\right|-\frac{1}{2} \sum_{f}\left|\phi_{i}-\operatorname{Re}\left(m_{f}\right)\right|
$$

are consistent with a prepotential $[1,2]$ :

$$
\begin{aligned}
{\left[\frac{1}{g_{5}^{2}}\right]_{i j} } & =\frac{\partial^{2} \mathcal{F}}{\partial \phi_{i} \partial \phi_{j}} \\
8 \pi^{2} \mathcal{F} & =\sum_{i=1}^{n_{c}}\left(\frac{h}{2} \phi_{i}^{2}+\frac{k_{\mathrm{cs}}}{6} \phi_{i}^{3}\right)+\frac{1}{12} \sum_{i, j=1}^{n_{c}}\left|\phi_{i}-\phi_{j}\right|^{3}-\frac{1}{12} \sum_{i=1}^{n_{c}} \sum_{f=1}^{n_{f}}\left|\phi_{i}-m_{f}\right|^{3} .
\end{aligned}
$$

This indicates SUSY extension from 4 supercharges in $4 \mathrm{D}$ to 8 supercharges in the continuum limit of the fifth dimension, which is a major ingredient of dimensional deconstruction. ${ }^{\langle 6\rangle}$ And most importantly, the deconstructed prepotential (4.49) is exactly as in eqs. (1.2-4) for the un-deconstructed $\mathrm{SQCD}_{5}$ (with a 'stringy' UV regulator which preserves all 8 supersymmetries), provided we identify the tree-level Chern-Simons coefficient as $k_{\mathrm{cs}}=n_{c}-\frac{1}{2} n_{f}-\Delta F$ according to eq. (3.27), and the tree-level inverse gauge coupling $h=\left(8 \pi^{2} / g_{5}^{2}\right)$ as $h=\operatorname{Re}(H) .\langle 7\rangle$

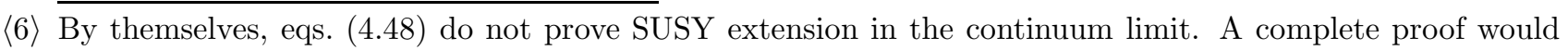
require calculating the Kähler function of the moduli fields and checking that the $\phi_{i}$ have the same metric as the gauge fields, and also that the Higgs moduli have the right metric and the right speed of light in the deconstructed dimension. Alas, we don't know how to calculate the non-perturbative Kähler function, so all we can do is hope that we may fine-tune it to agree with the extended SUSY. On the other hand, the gauge couplings are exactly calculable and cannot be fine-tuned, and that's why checking eqs. (4.48) is so important: If there is a prepotential, we may fine-tune the Kähler function to complete the SUSY extension; but if there is no prepotential, fine-tuning would not help.

$\langle 7\rangle$ Note that the tree level gauge coupling is invariant under the charge conjugation $\mathbf{C}$. That's why the same $H$ appears in both eqs. (4.28) and (4.45). 
We conclude this section by establishing the limits (if any) of the $h$ parameter space of the deconstructed $\mathrm{SQCD}_{5}$. Going back to eq. (4.24), we see that for $F<2 n_{c}, S$ - and hence $H$ - depends on $V$, and according to eq. (4.10) $V$ suffers from non-perturbative corrections when $\Delta F=0$. In the large quiver limit, eq. (4.8) becomes

$$
V \underset{L \rightarrow \infty}{\longrightarrow} \max \left(v_{1}, v_{2}\right)
$$

hence according to eq. (4.9)

$$
|V|^{2 n_{c}} \geq\left|v_{1}^{n_{c}} \times v_{2}^{n_{c}}\right|=\left|\Lambda^{2 n_{c}-F} \gamma^{F} \mu_{1} \mu_{2} \cdots \mu_{F}\right|
$$

regardless of the tree-level parameter $v$, and therefore for $\Delta F=0$,

$$
\left|\frac{V^{2 n_{c}-F}}{\gamma^{F} \Lambda^{2 n_{c}-F}}\right| \geq \prod_{f=1}^{F=n_{f}}\left|\frac{\mu_{f}}{V}\right| \Longrightarrow \operatorname{Re}(S) \geq \sum_{f=1}^{n_{f}} m_{f} .
$$

Hence, in light of eqs. (3.27) and (4.45),

$$
\text { for } k_{\mathrm{cs}}=+\left(n_{c}-\frac{1}{2} n_{f}\right), \quad h=\operatorname{Re}(H) \geq \frac{1}{2} \sum_{f=1}^{n_{f}} \operatorname{Re}\left(m_{f}\right) .
$$

On the other hand, for $\Delta F>0$ there is no lower limit on the magnitude of $V$; instead $V=v$ without any quantum corrections whatsoever. Hence, allowing for arbitrarily large or small $\Lambda / v$ ratios, we find that

$$
\text { for }\left|k_{\mathrm{cs}}\right|<\left(n_{c}-\frac{1}{2} n_{f}\right), \quad \text { all } h>-\infty \text { are allowed. }
$$

Finally, for $F=2 n_{c}$ eq. (4.24) does not depend on $V$ but only on the asymptotically-finite coupling $g^{2}$ of the $4 \mathrm{D}$ gauge theory $\left[S U\left(n_{c}\right)\right]^{L}$, hence $\operatorname{Re}(S) \geq 0$ and therefore,

$$
\text { for } k_{\mathrm{cs}}=-\left(n_{c}-\frac{1}{2} n_{f}\right), \quad h \geq-\frac{1}{2} \sum_{f=1}^{n_{f}} \operatorname{Re}\left(m_{f}\right) .
$$

Note that despite completely different 4D origins of the limits (4.53) and (4.55), in 5D these limits are related to each other by the charge conjugation $\mathbf{C}$. 
Formulæ (4.53-55) describe the complete range of the $h$ parameter of deconstructed $\mathrm{SQCD}_{5}$ for different values of quark masses and Chern-Simons levels. However, for

$$
h<h_{0}=\frac{1}{2} \sum_{f=1}^{n_{f}}\left|\operatorname{Re}\left(m_{f}\right)\right|
$$

the 5D inverse gauge couplings (4.46-47) become negative at the origin $\phi_{i} \equiv 0$ of the dynamical moduli space. Physically, this is quite impossible from both 5D and 4D points of view; indeed, the very existence of a spectral curve such as (4.2) guarantees that the $\operatorname{Im} \tau_{i j}(\varphi)$ matrix is positive definite ${ }^{\langle 8\rangle}$ for all moduli $\varphi_{i}$. In $4 \mathrm{D}$, this apparent paradox goes away when we remember that before deriving eqs. (4.46-47) we assumed a "sufficiently large" $\operatorname{Re}(S)$ to assure that the branching points $x_{1}, \ldots, x_{2 n_{c}}$ of the Riemann surface (4.21) come in close pairs (4.35), and it is precisely this assumption which fails for $h \leq h_{0}$. (Indeed, the inequalities (4.36) fail precisely when the right hand sides of eqs. (4.47) are no longer positive.) In the $h<h_{0}$ regime, the branching points $x_{1}, \ldots, x_{2 n_{c}}$ are arranged differently, and in $\S 6$ we shall see how such re-arrangements correspond to different phases of the 5D theory, separated from the "ordinary $\mathrm{SQCD}_{5}$ " phase by flop transitions [14] in the parameter/moduli space.

$\langle 8\rangle \overline{\text { By abuse of terminology, we call the } n_{c} \times n_{c}}$ matrix $\operatorname{Im} \tau_{i j}$ "positive definite" when we mean

$$
\sum_{i, j} \operatorname{Im} \tau_{i j} F_{i} F_{j}>0 \quad \forall F_{i} \text { such that } \sum_{i} F_{i}=0 .
$$




\section{Quantum Baryonic Branches}

In stringy UV completions of 5D SYM theories with $k_{\mathrm{cs}}=0(\theta=0$ for $S U(2))$ there is a peculiar Higgs branch, which connects to the Coulomb branch at the superconformal point $h=0, \phi_{i}=0 \forall i[2,8]$. In this section we shall see that the deconstructed $\mathrm{SYM}_{5}$ - as well as some $\mathrm{SQCD}_{5}$ theories - have similar Higgs branches. In quiver terms, they are exotic baryonic branches, where by exotic we mean that the baryonic VEVs involve 4D flavors with $\mu=0$ instead of the 5D flavors with $\mu=V e^{a m}$. Classically, such exotic branches do not exist, and even in the quantum theory they show up only at a particular value of the coupling parameter $H$, namely $H=0$ for $n_{f}=0$ and $\Delta F=n_{c}$.

But before we delve into quantum baryonic branches of the quiver, let us briefly review the Higgs branches of 5D SYM from the stringy point of view. A rather graphic picture of such branches obtains via the $(p, q)$-brane web construction of type IIB superstring [9] ${ }^{\langle 9\rangle}$ For $h>0$ the $S U\left(n_{c}\right)$ SYM webs look like
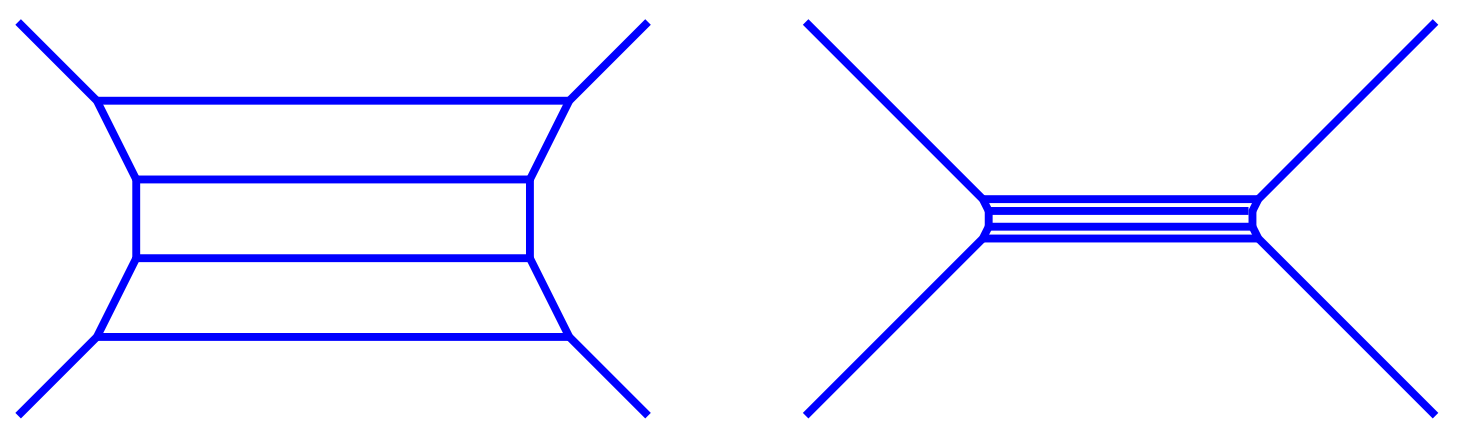

where the left web corresponds to the Coulomb branch with distinct $\phi_{i}$ and the right web to the unbroken $S U\left(n_{c}\right)$ point for $\phi_{i}=0 \forall i$ : a stack of $n_{c}$ coincident horizontal brane segments gives rise to non-abelian $S U\left(n_{c}\right)$ gauge symmetry. The inverse $5 \mathrm{D}$ coupling $h$ is proportional to the length of the stack, which depends on relative positions of the external legs. The directions of those external legs depend on the Chern-Simons level: for $k_{\mathrm{cs}}=0$ the legs diagonally across from each other are parallel, and for $h=0$ they line up in straight lines.

$\langle 9\rangle$ Briefly, there is a bunch of 5-branes, each spanning the $5 \mathrm{D}$ coordinates $X^{0}, \ldots, X^{4}$ and a segment of a real straight line in the $\left(X^{5}, X^{6}\right)$ plane; the segments form a web. A brane with $(\mathrm{D}, \mathrm{NS})$ charges $(p, q)$ is oriented according to $X^{5}+i X^{6}=\left(p+\tau_{s} q\right) \times$ real + const; where $\tau_{s}$ is the complex type IIB coupling; this condition provides for 8 unbroken supercharges in 5D. The brane joints in the web are governed by the zero-force condition, which is equivalent to the $(p, q)$ charge conservation. Please see $[9,11,12]$ for more details. 
Hence, when all the brane boxes in the middle of the web collapse to a point for $\phi_{i}=0 \forall i$, the external legs can re-connect as two intersecting infinite branes:

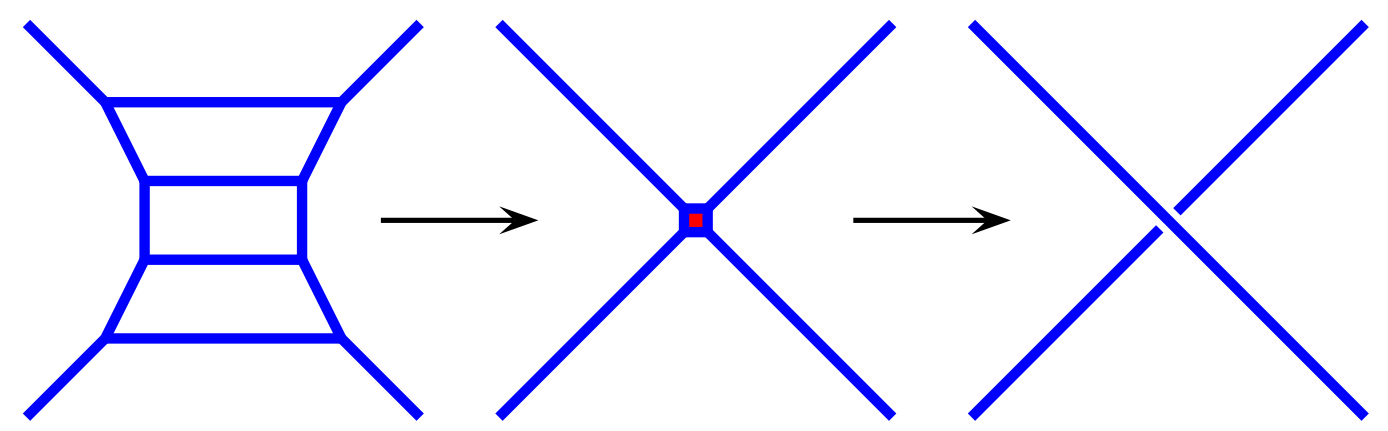

Pulling the two reconnected branes apart (in a direction perpendicular to both branes) corresponds to the Higgs branch of the 5D SYM. Note that this branch exists only for $k_{\mathrm{cs}}=0$ : for other Chern-Simons levels the external legs cannot reconnect because they are not parallel.

In M theory construction of 5D SYM theories there are similar Higgs branches for $k_{\mathrm{cs}}=0$ only (or $\theta=0$ for $S U(2)$ ). To obtain an $S U\left(n_{c}\right) \mathrm{SYM}_{5}$, we compactify $\mathrm{M}$ theory on a Calabi-Yau sixfold with a conical singularity of the CY, where the cone's base is a $Y^{p, q}$ Sazaki-Einstein space with $p=n_{c}$ and $q=k_{\mathrm{cs}}[34,35]$. Or rather, the superconformal point obtains from such a singularity while the Coulomb branch corresponds to resolutions of its Kähler structure. For $q=0$ (and only $q=0$ ) we may also deform the complex structure of the singularity, which requires keeping the Kähler structure unresolved; in field theory this corresponds to a Higgs branch with frozen Coulomb parameters/moduli $h=0$ and $\phi_{i}=0 \forall i$. The reason cones over $Y^{p, 0}$ are special in this way is that they are $\mathbb{Z}_{p}$ orbifolds of the conifold, whose complex structure has one deformation mode; this mode is $\mathbb{Z}_{p}$ invariant $\forall p$, and so it's inherited by the orbifolds. Other cones with $q \neq 0$ have rigid complex structures which cannot be deformed; consequently, SYM theories with $k_{\mathrm{cs}} \neq 0$ do not have Higgs branches.

The goal of this section is to deconstruct the Higgs branch of SYM with $k_{\mathrm{cs}}=0-$ as well as similar Higgs branches in some strongly-coupled $\mathrm{SQCD}_{5}$ theories — via exotic baryonic branches of the quiver theory. But first, we need to look at the ordinary baryonic branches of a quiver with generic $n_{c}, n_{f}$, and $\Delta F$. As discussed at the end of $\S 2$, classical baryonic branches have squark and antisquark VEVs as in eq. (2.48) (modulo a flavor symmetry), 
and their existence requires $n_{c}$ zero modes in the quarks' mass matrix, thus eq. (2.43) for the Coulomb moduli $\varphi_{i}$. Actually, (2.43) is an overdetermined system of $n_{c}$ equations for $n_{c}-1$ independent moduli, hence a baryonic branch exists only if $m_{1}+\cdots+m_{n_{c}}=0$ (modulo $2 \pi i / L a)$, or in $4 \mathrm{D}$ terms, if $\mu_{1}^{L} \times \cdots \times \mu_{n_{c}}^{L}=V^{L n_{c}}$. Of course this is all modulo a flavor symmetry, so in general a baryonic branch with flavors $f_{1}, \ldots, f_{n_{c}}$ exists if and only if

$$
\prod_{f \in \text { Baryon }} \mu_{f}^{L}=V^{L n_{c}}
$$

Obviously this condition excludes $4 \mathrm{D}$ flavors with $\mu=0$, so classical baryonic branches involve only the $5 \mathrm{D}$ flavors with $\mu=V e^{a m} \neq 0$.

In the quantum theory we cannot look at individual squark VEVs; instead, we analyze VEVs of gauge-invariant chiral operators. In our 4D paper [25] we found that the offshell chiral ring of the quiver theory contains a whole zoo of baryon-like operators, but in the on-shell ring, they are all related to each other via equations of motion (AKA Konishi anomaly equations). Consequently, for each choice of $n_{c}$ distinct flavors, there is at most one independent baryonic VEV (and likewise, one antibaryonic VEV). And similar to the classical theory, such (anti)baryonic VEVs over-determine the Coulomb moduli, although eq. (5.3) is corrected by instanton effects. The exact constraint is best stated in terms of the spectral curve (4.2) of the quiver: A baryonic branch exists when the curve has no branch cuts at all; instead, it factorizes according to

$$
Y^{2}-P(X) \times Y+(-1)^{F} \alpha B(X)=\left(Y-B_{1}(X)\right) \times\left(Y-(-1)^{F} \alpha B_{2}(X)\right)=0
$$

where

$$
B_{1}(X)=\prod_{f \in \text { Baryon }}\left(X-\mu^{L}\right) \quad \text { of degree }=n_{c}
$$

encodes masses of flavors involved in the baryonic VEV, and

$$
B_{2}(X)=\prod_{f \notin \text { Baryon }}\left(X-\mu^{L}\right) \quad \text { of degree }=F-n_{c}
$$

encodes masses of the remaining flavors. Factorization of the spectral curve implies that the 
link resolvent $T(X)$ (cf. eqs. (4.12-14)) has poles instead of branch cuts:

$T(X) \stackrel{\text { def }}{=}\left\langle\operatorname{Tr} \frac{1}{X-\Omega_{L} \cdots \Omega_{1}}\right\rangle=\frac{1}{Y} \frac{\partial Y}{\partial X}= \begin{cases}\sum_{f \in \text { Baryon }} \frac{1}{X-\mu_{f}^{L}} & \text { on the physical sheet, } \\ \sum_{f \notin \text { Baryon }} \frac{1}{X-\mu_{f}^{L}} & \text { on the unphysical sheet. }\end{cases}$

Note that the poles differ between the two Riemann sheets, and consequently the Coulomb moduli $\varpi_{i}=V^{L} e^{L a \varphi_{i}}$ do not match the poles; instead, eq. (5.4) implies

$$
P(X) \equiv \prod_{i=1}^{n_{c}}\left(X-\varpi_{i}\right)=B_{1}(X)+(-1)^{F} \alpha \times B_{2}(X)
$$

Physically, the first term on the right hand side reproduces the classical eq. (5.3) for the moduli of a baryonic branch, while the second term is the quantum correction; it arises at the one-diagonal-instanton level (one instanton in the $S U\left(n_{c}\right)_{\text {diag }}$, i.e. one instanton in each $S U\left(n_{c}\right)_{\ell}$ factor). Eq. (5.7) over-determines the moduli: in order to maintain the product constraint (4.7), the masses must satisfy

$$
\prod_{f \in \text { Baryon }} \mu_{f}^{L}+(-1)^{F} \alpha \times \prod_{f \notin \text { Baryon }} \mu_{f}^{L}=V^{L n_{c}}
$$

Unlike its classical analogue (5.3), this formula involves masses of all $F$ flavors rather than just the $n_{c}$ flavors involved in the baryonic VEVs. In particular, it involves flavors with $\mu=0$, if any, and this gives rise to two distinct types of baryonic branches: they either contain none of the $\mu=0$ flavors, or else they contain all of them at once.

Ordinary baryonic branches are of the first type: all $n_{c}$ flavors have $\mu=V e^{a m} \neq 0$ and are visible in 5D. For $\Delta F>0$, eq. (5.9) for such branches reduces to the classical formula (5.3), or in $5 \mathrm{D}$ terms $\sum_{f \in \text { Baryon }} m_{f}=0$. However, the moduli $\varphi_{i}$ suffer quantum corrections according to

$$
\prod_{i=1}^{n_{c}}\left(x-e^{L a \varphi_{i}}\right)=\prod_{f \in \text { Baryon }}\left(x-e^{\operatorname{Lam}_{f}}\right)+(-1)^{F} e^{-L a S} \times x^{\Delta F} \times \prod_{f \notin \text { Baryon }}\left(x-e^{\operatorname{Lam}_{f}}\right) .
$$

In the decompactification limit $L a \rightarrow \infty$, the second term on the right hand side becomes neg- 
ligible if the coupling is weak enough; in this regime we recover the classical constraint (2.43). On the other hand, for strong coupling the second term remains important even in the 5D limit; we shall see an example of such quantum shift of the baryonic branch in $§ 6.3$.

Baryonic branches of the second type are exotic: they involve $\mu=0$ flavors, indeed all $\Delta F$ of them, plus $\left(n_{c}-\Delta F\right) 5 \mathrm{D}$ flavors to complete the baryon. Clearly, such branches require $F=n_{f}+\Delta F \geq n_{c}$ but $\Delta F \leq n_{c}$; in $5 \mathrm{D}$ terms this amounts to a constraint on the Chern-Simons level:

$$
\text { Exotic baryons exist only for }\left|k_{\mathrm{cs}}\right| \leq \frac{n_{f}}{2} \text {. }
$$

In particular, in SYM theories with $n_{f}=0$, the exotic baryonic branches exist only for $k_{\mathrm{cS}}=0\left(\theta=0\right.$ for $\left.n_{c}=2\right)$.

For an exotic baryonic branch, eq. (5.9) depends on the coupling $\alpha$ as much as on the masses $\mu_{f}$; solving it for $\alpha$ gives us

$$
(-1)^{F} \alpha=\left[\frac{V^{n_{c}}}{\prod_{f \notin \text { Baryon }} \mu_{f}}\right]^{L}
$$

or in terms of $S$ and $H$ parameters ( $c f$. eqs. (4.24) and (4.28))

$$
S=\sum_{f \notin \text { Baryon }}^{5 \mathrm{D} \text { only }} m_{f} \Longrightarrow H=\sum_{f \notin \text { Baryon }}^{5 \mathrm{D} \text { only }} \frac{m_{f}}{2}-\sum_{f \in \text { Baryon }}^{5 \mathrm{D} \text { only }} \frac{m_{f}}{2}
$$

In particular, for a SYM theory with $k_{\mathrm{cs}}=0$, the exotic branch requires $H=0$. For other values of the coupling $H$ - either too weak or too strong - exotic baryons cannot develop VEVs. This behavior is in perfect agreement with the Higgs branch of the brane web (5.2): To pull the web apart in the out-of-plane direction, the external legs of the web must be perfectly aligned, which means $h=0$ exactly, no more and no less.

The $\varphi_{i}$ moduli of an exotic baryonic branch are constrained by

$$
\prod_{i=1}^{n_{c}}\left(x-e^{L a \varphi_{i}}\right)=x^{\Delta F} \times \prod_{f \in \text { Baryon }}^{5 \mathrm{~d}}\left(x-e^{\operatorname{Lam}_{f}}\right)+(-1)^{F} e^{-L a S} \prod_{f \notin \text { Baryon }}^{5 \mathrm{~d}}\left(x-e^{\operatorname{Lam}_{f}}\right)
$$


or in light of eq. (5.13),

$$
\prod_{i=1}^{n_{c}}\left(x-e^{L a \varphi_{i}}\right)=x^{\Delta F} \times \prod_{f \in \text { Baryon }}^{5 \mathrm{~d}}\left(x-e^{\text {Lam }_{f}}\right)+(-1)^{F} \prod_{f \notin \text { Baryon }}^{5 \mathrm{~d}}\left(x \times e^{- \text {Lam }_{f}}-1\right) .
$$

Note that both terms on the second line here are of comparable magnitudes and both remain important in the decompactification limit. In particular, for $n_{f}=0$ and $\Delta F=n_{c}$ (the SYM theory with $k_{\mathrm{cs}}=0$ ) the first term becomes $x^{n_{c}}$ while the second term is \pm 1 . Together, they provide for

$$
\prod_{k=1}^{n_{c}}\left(x-e^{L a \varphi_{k}}\right)=x^{n_{c}} \pm 1 \Longrightarrow L a \varphi_{k}=2 \pi i \times k \quad \text { or } \quad 2 \pi i \times\left(k-\frac{1}{2}\right), \quad k=1, \ldots, n_{c}
$$

or in 5D terms, all $\phi_{k}=0$. Together with the $h=0$ condition, this brings us to the superconformal point where the exotic baryonic Higgs branch meets the Coulomb branch. Again, this is in perfect agreement with the string theory: in terms of the brane web (5.2), aligning the external legs (setting $h=0$ ) is not enough, one must also collapse all the cycles (by setting all $\phi_{k}=0$ ) before pulling the web apart (i.e., turning on a hypermultiplet VEV).

In [25] we rejected the exotic baryonic branches because of their link resolvents: according to eq. (5.7), when a baryonic branch involves a $\mu=0$ flavor, $T(X)$ has a pole at $X=0$ on the physical sheet. This implies that the product $\Omega_{L} \cdots \Omega_{1}$ of link fields has a zero eigenvalue - or rather $\Delta F$ zero eigenvalues, judging by the residue of the pole at $X=0$ - and we thought that to be impossible since all the $\Omega_{\ell}$ matrices are invertible. In retrospect, that was a mistake.

Indeed, in the quantum theory $\left\langle\operatorname{det}\left(\Omega_{L} \cdots \Omega_{1}\right)\right\rangle \neq \prod_{\ell}\left\langle\operatorname{det}\left(\Omega_{\ell}\right)\right\rangle$, and in [25] we have actually calculated the quantum corrections to $\left\langle\operatorname{det}\left(\Omega_{L} \cdots \Omega_{1}\right)\right\rangle$ arising from instantons in the diagonal $S U\left(n_{c}\right)_{\text {diag }}$ as well as instantons individual $S U\left(n_{c}\right)_{\ell}$ factors. But somehow, we overlooked the possibility that the quantum corrections may cancel the classical contribution and lead to $\left\langle\operatorname{det}\left(\Omega_{L} \cdots \Omega_{1}\right)\right\rangle=0$ despite invertibility of the individual $\Omega_{\ell}$ matrices. For an example of such cancellation, consider a quiver with $F=n_{c}$, in which case

$$
\left\langle\operatorname{det}\left(X-\Omega_{L} \cdots \Omega_{1}\right)\right\rangle=P(X)-(-1)^{n_{c}} \times \alpha
$$


(cf. [25] for details), and hence

$$
\left\langle\operatorname{det}\left(\Omega_{L} \cdots \Omega_{1}\right)\right\rangle=V^{L n_{c}}-\alpha=V^{L n_{c}} \times\left(1-e^{-L a H}\right) \rightarrow 0 \text { for } H=0 .
$$

More generally, for a quiver with $F>n_{c}$ the characteristic polynomial of the link product is given by

$$
\left\langle\operatorname{det}\left(X-\Omega_{L} \cdots \Omega_{1}\right)\right\rangle=\text { Polynomial part of }\left[\frac{P(X)+\sqrt{P^{2}(X)-4(-1)^{F} \alpha B(X)}}{2}\right] \text {. }
$$

When the quiver has a baryonic branch - ordinary or exotic - and the spectral curve factorizes according to eq. (5.4), eq. (5.19) reduces to

$$
\left\langle\operatorname{det}\left(X-\Omega_{L} \cdots \Omega_{1}\right)\right\rangle= \begin{cases}B_{1}(X) & \text { on the physical sheet, } \\ (-1)^{F} \alpha B_{2}(X) & \text { on the unphysical sheet }\end{cases}
$$

in perfect agreement with the link resolvent (5.7) of a baryonic branch. In particular, for an exotic branch, the determinant (5.20) has precisely $\Delta F$ zero eigenvalues, which agrees with $T(X)$ having a pole of residue $\Delta F$ at $X=0$ on the physical sheet.

To summarize, quiver theories with $\Delta F \leq n_{c} \leq \Delta F+n_{f}$ (which correspond to $\mathrm{SQCD}_{5}$ with $\left|k_{\mathrm{cs}}\right| \leq \frac{n_{f}}{2}$ ) have exotic baryonic branches involving $\mu=0$ flavors. Such branches exist only for specific values of the coupling $H$ as well as of the Coulomb moduli $\varphi_{i}$; in particular, for the SYM with $k_{\mathrm{cs}}=0$ (or $\theta=0$ for $n_{c}=2$ ), the exotic baryonic branch grows out of the $5 \mathrm{D}$ superconformal point $h=0, \phi_{i}=0 \forall i$. In string implementations of the same 5D SYM, the existence of a Higgs branch at the superconformal point is well-known, but its baryonic nature is a novel result. 


\section{6. $S U(2)$ Examples of Phase Structures and Flop Transitions.}

In this section we present four examples of deconstructive quiver theories and study their phases for $h=\frac{8 \pi^{2}}{g_{5 \mathrm{~d}}^{2}}>0$ and $h<0$. In $4 \mathrm{D}$ terms, negative $h$ means $|\Lambda|>|V|$ and hence strongly coupled $S U(2)_{\ell}$ factors of the quiver theory at the $4 \mathrm{D} \rightarrow 5 \mathrm{D}$ threshold $1 / a$. However, thanks to unbroken $\mathcal{N}=1$ SUSY in $4 \mathrm{D}$, the holomorphic spectral curve (4.21) remains nonperturbatively exact despite the strong coupling, and thus may be used for deconstructing the exotic phases of $5 \mathrm{D}$ theories.

Our presentation here is quite detailed, which makes for a rather looooong section. So let us state our main result upfront: in all examples, the deconstructed $\mathrm{SQCD}_{5}$ has exactly the same phase diagram as the string-theoretical UV completion of the same 5D theory. And now, the readers who are not interested in technical details may skip over many formulae in this section and just look at the phase diagrams themselves; they appear on pages 59, 67, $75-76,82,85$, and 87.

For simplicity, all our examples have $n_{c}=2$ and hence Coulomb branches with only one abelian gauge coupling, and also only one independent modulus $\varphi=\varphi_{2}=-\varphi_{1}$; without loss of generality we assume $\phi \equiv \operatorname{Re} \varphi>0$. In $4 \mathrm{D}$, the quiver theory with $n_{c}=2$ has an elliptic rather than hyperelliptic spectral curve (4.21), with four branching points $x_{1}, x_{2}, x_{3}, x_{4}$ located at roots of the quartic equation

$$
D(x) \equiv\left(x^{2}-2 \cosh (\operatorname{La\varphi }) \times x+1\right)^{2}-4(-1)^{F} e^{-L a S} \times x^{\Delta F} \prod_{f=1}^{n_{f}}\left(x-e^{\text {Lam }_{f}}\right)=0
$$

The $4 \mathrm{D}$ abelian gauge coupling $\tau$ — or rather its invariant

$$
j(\tau) \equiv j\left(\frac{a \tau+b}{c \tau+d}\right)=e^{-2 \pi i \tau}+744+\text { a convergent power series in } e^{+2 \pi i \tau}
$$

under electric-magnetic duality — follows from the cross-ratio

$$
\chi=\frac{\left(x_{1}-x_{4}\right)\left(x_{2}-x_{3}\right)}{\left(x_{1}-x_{2}\right)\left(x_{3}-x_{4}\right)}
$$


of the branching points according to

$$
j(\tau)=-256 \frac{\left(\chi^{2}+\chi+1\right)^{3}}{\chi^{2}(\chi+1)^{2}}
$$

A finite 5D coupling corresponds to $\operatorname{Im} \tau \propto L a$ and hence exponentially large $j$; in terms of the cross ratio (6.3), this means $\chi \rightarrow 0, \chi \rightarrow-1$, or $\chi \rightarrow \infty$, with

$$
\operatorname{Re} \log \left(\frac{16}{\chi}, \frac{16}{\chi+1}, \text { or }(16 \chi)\right)=\frac{L a \times T}{2} \quad \text { where } \quad T \stackrel{\text { def }}{=} \frac{8 \pi^{2}}{g_{5 d}^{2}[U(1)]}
$$

Our first two examples have $n_{f}=0$ in $5 \mathrm{D}$, but the $4 \mathrm{D}$ quivers do have $\mu=0$ quarks, $\Delta F=1$ in the first example $(\S 6.1)$ and $\Delta F=2$ in the second example $(\S 6.2) \cdot{ }^{\langle 10\rangle}$ In $5 \mathrm{D}$, both examples yield SYM theories for $h>0$; according to eq. (3.30), the first SYM has vacuum angle $\theta=\pi$ while the second has $\theta=0$. But we shall see that the $h<0$ regimes of the two examples are very different: the $\Delta F=1$ model has two distinct Coulomb phases the SYM phase and the $E_{0}$ phase - separated by a flop transition, while the $\Delta F=2$ model has only one Coulomb phase, but it also has a Higgs phase (which we deconstruct as the exotic baryonic branch). In string theory constructions, these two 5D theories are properly known as the $D_{0}($ for $\theta=0)$ and the $\tilde{D}_{0}\left(\right.$ for $\theta=\pi$ ), although they are often called $E_{1}$ and $\tilde{E}_{1}$ after their respective superconformal limits at $h=\phi=0$ [2]. In this article however, we call them $D_{0}$ and $\tilde{D}_{0}$ because we focus on deconstructing the non-conformal Coulomb and Higgs phases of the two theories.

In $\S 6.3$ we present two more $S U(2)$ models, this time with two quark flavors in 5D. For simplicity, we restrict our analysis to equal masses (modulo sign) for the two flavors. The first model has $m_{1}=-m_{2}$ and $\Delta F=1$ while the second has $m_{1}=m_{2}$ and $\Delta F=0$; we present them together in $\S 6.3$ because their spectral curves are dual to each other. However, the quiver theories themselves are not dual, and even their moduli spaces are not quite dual. In particular, the $\Delta F=1$ model has more Higgs branches than the $\Delta F=0$ model.

$\langle 10\rangle \overline{\text { There is also a distinct theory with } \Delta F=0}$; it was studied in much detail in [20]. But this theory has $h \geq 0$ only, and it does not have any phase transitions at all. 


\subsection{The $\widetilde{D}_{0}$ MOdel: $n_{c}=2, n_{f}=0, \Delta F=1$.}

We begin with the flavorless $S U(2)$ model with $\theta=\pi$, or in $4 \mathrm{D}$ quiver terms, $n_{c}=2$, $n_{f}=0$, and $\Delta F=1$. The spectral curve of this model has branching points at roots of the discriminant

$$
D(x) \equiv\left(x^{2}-2 \cosh (\operatorname{La\varphi }) \times x+1\right)^{2}+4 e^{-L a H} \times x=0 .
$$

There is no simple general formula for these roots, but in the $L a \rightarrow \infty$ limit there are simple approximations for various regimes of $h=\operatorname{Re}(H)$ and $\phi=\operatorname{Re}(\varphi)$. As a warm up exercise, let us start with the $h>0$ regime and reproduce the Seiberg's formula [1] for $S U(2)$ :

$$
T \equiv \frac{8 \pi^{2}}{g_{5 d}^{2}[U(1)]}=2 h+\left(8-n_{f}\right) \times \phi .
$$

For $h>0$ and also $h, \phi \gg \frac{1}{L a}$, the four roots of eq. (6.6) lie approximately at

$$
x_{1,2} \approx e^{+L a \varphi} \pm 2 i e^{-L a(H+\varphi) / 2}, \quad x_{3,4} \approx e^{-L a \varphi} \pm 2 i e^{-L a(H+3 \varphi) / 2}
$$

and have a large cross-ratio (6.3)

$$
\chi \approx \frac{-1}{16} \times e^{L a(H+4 \varphi)}
$$

Hence, according to eq. (6.5)

$$
T=2 h+8 \phi
$$

in perfect agreement with the Seiberg's formula (6.7).

Another useful cross-check of the $h>0$ phase is to reproduce the $S U(2)$ restoration in $5 \mathrm{D}$ for $\phi \rightarrow 0$. In $4 \mathrm{D}$ terms, classical $S U(2)$ restoration happens for $L a \varphi=0$ or $\pi i$ (modulo $2 \pi i$ ); the two allowed values of the Wilson line are due to $W^{ \pm}$particles having charges \pm 2 in fundamental (quark) charge units. Moreover, quantum effects in a classicallyunbroken $S U(2)$ SYM split a single singularity into a close pair of Seiberg-Witten points where magnetic monopoles or dyons become massless and $j(\tau)$ has a pole. In Seiberg-Witten terms, $\operatorname{tr}\left((\text { adjoint scalar })^{2}\right)$ corresponds to $(L a \varphi)^{2}$ or $(L a \varphi-\pi i)^{2}$ while the strong-coupling 
scale $\Lambda_{\mathrm{SW}}^{4}$ of the $5 \mathrm{D}$ theory compactified to $4 \mathrm{D}$ (i.e., the diagonal $S U(2)$ of the quiver) corresponds to $\pm e^{-L a H}$. Hence, we expect $j(\tau(\varphi))$ to have poles at

$$
(L a \varphi)^{2}= \pm O\left(e^{-L a H / 2}\right) \quad \text { and } \quad(L a \varphi-\pi i)^{2}= \pm O\left(e^{-L a H / 2}\right)
$$

In terms of the spectral curve, a pole of $j$ means that two of the branching points $x_{1}, x_{2}, x_{3}, x_{4}$ collide with each other. To see how it happens in the our model, let's take the $\sinh ^{2}(\operatorname{La} \varphi) \rightarrow 0$ limit of eq. (6.6). In this limit, all four branching points cluster around \pm 1 (depending on the sign of $\cosh (\operatorname{La} \varphi))$, so to resolve the situation, we shift and rescale

$$
\begin{aligned}
x & =x^{\prime} \times \sinh (\operatorname{La\varphi })+\cosh (\operatorname{La\varphi }) \\
D^{\prime}(x) & =\frac{D(x)}{\sinh ^{4}(\operatorname{La\varphi })} \\
& \approx\left(x^{\prime 2}-1\right)^{2} \pm 4 \frac{e^{-L a H}}{\sinh ^{4}(\operatorname{La\varphi })} .
\end{aligned}
$$

The rescaled discriminant has a double root when the second term on the last line above equals to -1 , or in $\varphi$ terms when

$$
(L a \varphi)^{2} \approx \pm 2 i e^{-L a H / 2} \quad \text { or } \quad(L a \varphi-\pi i)^{2} \approx \pm 2 e^{-L a H / 2}
$$

in perfect agreement with eq. (6.11). ${ }^{\langle 11\rangle}$ Thus, we conclude that the $\phi \rightarrow 0$ limit in the $h>0$ regime of the quiver theory properly deconstructs the $S U(2)$ restoration in $5 \mathrm{D}$.

Now let us consider the negative $h$ regime of the theory. In this regime, the roots of eq. (6.6) form three different patterns depending on the ratio of $\phi$ to $-h$ :

- For $\phi>(-h)>0$, the pattern is similar to the $h>0$ regime, and the roots lie at

$$
x_{1,2} \approx e^{+L a \varphi} \pm 2 i e^{-L a(H+\varphi) / 2}, \quad x_{3,4} \approx e^{-L a \varphi} \pm 2 i e^{-L a(H+3 \varphi) / 2} .
$$

$\langle 11\rangle$ Note that the $\varphi$ moduli space in $4 \mathrm{D}$ is a half-cylinder: besides the $\varphi \equiv \varphi+(2 \pi i / L a)$ redundancy of the Wilson line, we also identify $\varphi \equiv-\varphi$ because of the symmetry between the two eigenvalues $\varphi_{1}=-\varphi_{2}$. This space has two $\mathbb{Z}_{2}$ orbifold singularities at $L a \varphi=0$ or $\pi i$, and the proper single-valued coordinates near these points are respectively $(L a \varphi)^{2}$ and $(L a \varphi-\pi i)^{2}$. Therefore, eq. (6.14) describes four singularities rather then eight. 
Consequently, the cross ratio (6.3) is $\chi=\frac{-1}{16} e^{L a(H+4 \varphi)}$, and the $5 \mathrm{D}$ inverse coupling is

$$
T=2 h+8 \phi
$$

exactly as for $h>0$.

- For $\phi<(-h)$ but $\phi>(-h / 3)>0$, the pattern is slightly different:

$$
\begin{aligned}
x_{1,2} & \approx e^{+L a \varphi} \pm 2 i e^{-L a(H+\varphi) / 2}, \\
\text { but } x_{3} & \approx-2 e^{-L a(H+2 \varphi)}, \\
\text { and } x_{4} & \approx-\frac{1}{2} e^{+L a H} .
\end{aligned}
$$

In this case, the cross ratio is $\chi=\frac{i}{8} e^{L a(3 H+9 \varphi) / 2}$, and hence the 5D inverse coupling is

$$
T=3 h+9 \phi
$$

- Finally, for $0<\phi<(-h / 3)$ we have a very different pattern of one small root and three large roots equidistant from each other:

$$
\begin{gathered}
x_{1,2,3} \approx-\sqrt[3]{4} e^{2 \pi i k / 3} \times e^{-L a H / 3}+\frac{2}{3} e^{L a \varphi}, \\
k=1,2,3 \\
x_{4} \approx-\frac{1}{4} e^{+L a H} .
\end{gathered}
$$

For this pattern, the cross-ratio is $\chi \approx e^{-2 \pi i / 3}-2^{1 / 3} 3^{-1 / 2} i e^{L a(3 \varphi+H) / 3}$, and hence

$$
j(\tau) \approx 512 e^{+L a(H+3 \varphi)} \ll 1
$$

Such small $j$ indicates strong rather than weak 4D gauge coupling: $\tau$ asymptotes to a self-dual point $e^{2 \pi / 3}$ (a corner of the Teichmuller space) and stops depending on $\varphi$ as long as $\phi<(-h / 3)$. In 5D terms, such strong $\tau$ deconstructs to

$$
T=\frac{\sqrt{3} /(4 \pi)}{L a} \approx 0
$$

i.e., $g_{5 D}^{2}[U(1)]=O(L a)$ and becomes infinite in the decompactification limit. 
Let us summarize the various regimes of the deconstructed $T(\phi, h)$ behavior in one picture:

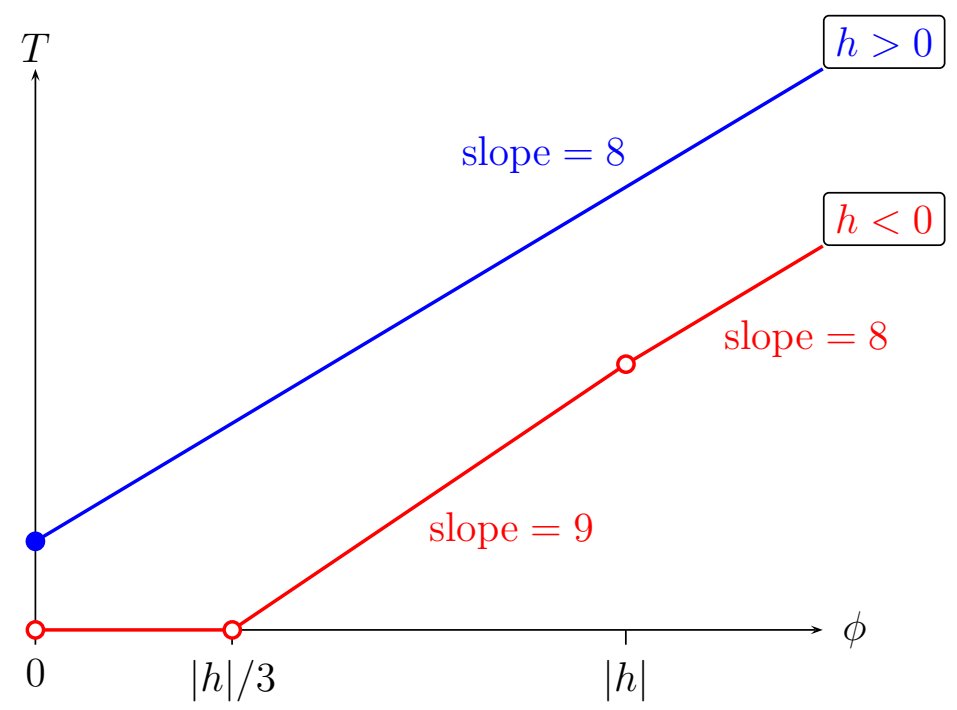

The blue and red line here plot $T(\phi)$ for fixed values of $h$. The blue line is for the $h>0$ regime, and the solid blue circle at its end indicates $S U(2)$ restoration in $5 \mathrm{D}$ for $\phi=0$. The red line is for the $h<0$ regimes, and the open red circles indicate regime changes at $\phi=(-h), \phi=(-h) / 3$, and maybe $\phi=0$; we shall investigate them momentarily.

Let us start with the right circle and take a closer look at the spectral curve of the deconstructed theory for $\varphi=-H+O(1 / L a)$. In this regime, two roots of the curve's discriminant (6.6) as in eqs. (6.15) and (6.17),

$$
x_{1,2} \approx e^{+L a \varphi} \pm 2 i e^{-L a(H+\varphi) / 2}
$$

while the other two roots $x_{3}, x_{4}$ satisfy a quadratic equation

$$
\left(e^{L a \varphi} x\right)^{2}-2\left(1-2 e^{-L a(\varphi+H)}\right) \times\left(e^{L a \varphi} x\right)+1=0
$$

and collide with each other for $\operatorname{La}(\varphi+H)=0$ (modulo $2 \pi i)$. This collision creates a pole in $j(\tau)$, indicating a charged particle becoming massless at this point in the moduli space. Since the $4 \mathrm{D}$ coupling $\tau$ is generally very weak in this area, we conclude that the massless particle's charge is electric rather than magnetic. Also, a single pole at a unique (modulo $2 \pi$ ) value of the Wilson line indicates the charge is \pm 1 in fundamental units. In other words, 
the massless $4 \mathrm{D}$ particle is a quark, and it deconstructs a 5D quark which (in the $h<0$ phase) becomes massless at $\phi=(-h)$.

Note that the 4D quiver of our model does have quarks, but perturbatively they have no light modes (with masses $\ll(1 / a)$ ) and thus decouple from the 5D physics. Apparently, when the quiver theory is strongly coupled for $h<0$, those quarks somehow become light and show up in 5D. In other words, the $h<0$ phase of the deconstructed theory has a quark flavor that the $h>0$ "ordinary $\mathrm{SYM}_{5}$ " phase does not know about.

Now consider the left red circle at $\phi=0$. In the $h<0$ regime, the spectral curve does not degenerate when $L a \varphi$ becomes small. Instead, the four roots of the discriminant (6.6) form the same pattern (6.19) as for $\phi>0$ (but $\phi<(-h) / 3$ ), and nothing special happens for $L a \varphi=O(1)$. In $5 \mathrm{D}$ terms, there is no $5 \mathrm{D} S U(2)$ restoration in the $h<0$ phase for $\phi=0$, nor anything else special at this point.

But after this disappointment, the middle red circle at $\phi=(-h) / 3$ turns out to be very interesting. Looking at the spectral curve's discriminant for $3 \varphi+H=O(1 / L a)$, we find one small root $x_{4} \approx \frac{-1}{4} e^{+L a H}$ and three big roots $x_{1,2,3}$ governed by a cubic polynomial

$$
\left(e^{-L a \varphi} x\right)^{3}-2\left(e^{-L a \varphi} x\right)^{2}+\left(e^{-L a \varphi} x\right)+4 e^{-L a(3 \varphi+H)}=0
$$

For large $e^{-L a(3 \varphi+H)}$ these roots form an equilateral triangle as in eq. (6.19), while for small $e^{-L a(3 \varphi+H)}$ the $x_{3}$ root is much smaller than the $x_{1,2}$ roots as in eq. (6.17). And for an intermediate value of $e^{-L a(3 \varphi+H)}=\frac{-1}{27}$, two of the roots collide and the spectral curve degenerates. In $L a \varphi$ terms, the degeneration happens at three distinct points

$$
L a \varphi_{k}=-\frac{L a H}{3}+\log 3+\frac{2 \pi i\left(k-\frac{1}{2}\right)}{3} \quad(\text { modulo } 2 \pi i), \quad k=1,2,3
$$

Moreover, at each point the degeneration is due to collision of a different pair of roots, which leads to distinct, non-commuting monodromies around each point. In physical 4D terms, this means singularities due to massless particles of different charge types: electric, magnetic, and dyonic. And reconstructing this behavior in terms of a 5D theory compactified on a large circle calls for a nontrivial superconformal theory in 5D. 
Specifically, this pattern of three singularities related by $2 \pi i / 3$ Wilson lines is characteristic of compactified 5D SCFT known as the $E_{0}$. Unlike the other SCFTs in the $E_{n}$ series which obtain in the $h \rightarrow 0$ limits of $S U(2)$ gauge theories with $n-1$ massless flavors, the $E_{0}$ is an isolated SCFT. It has a dynamical modulus field $\hat{\phi}$, but it does not have any nondynamical parameters (like $h$ or quark masses) one needs to tune to obtain superconformal behavior for $\hat{\phi}=0$. In $5 \mathrm{D}, \hat{\phi}$ is a real field which takes non-negative values only, but after compactification to $4 \mathrm{D}$, it becomes a cylindrical complex variable $\hat{\varphi}$ whose real part could be either positive or negative. However, for negative $\operatorname{Re} \hat{\varphi}$ the $4 \mathrm{D}$ gauge coupling $\tau$ asymptotes to a selfdual point — which corresponds to infinite 5D coupling — while for positive Re $\hat{\varphi}$ the $4 \mathrm{D}$ coupling is weak, $\operatorname{Im} \tau \propto 2 \pi R$, which indicates finite coupling in $5 \mathrm{D}$, specifically $T=9 \hat{\phi}$. Comparing this behavior to our quiver theory with $h<0$ and $\phi \approx(-h) / 3$, we immediately see that the $5 \mathrm{D}$ theory here is the $E_{0} \mathrm{SCFT}$ whose Coulomb modulus can be identified as $\hat{\phi}=\phi-\frac{1}{3}(-h)$.

Altogether, at this point we may complete the figure (6.22) as follows:

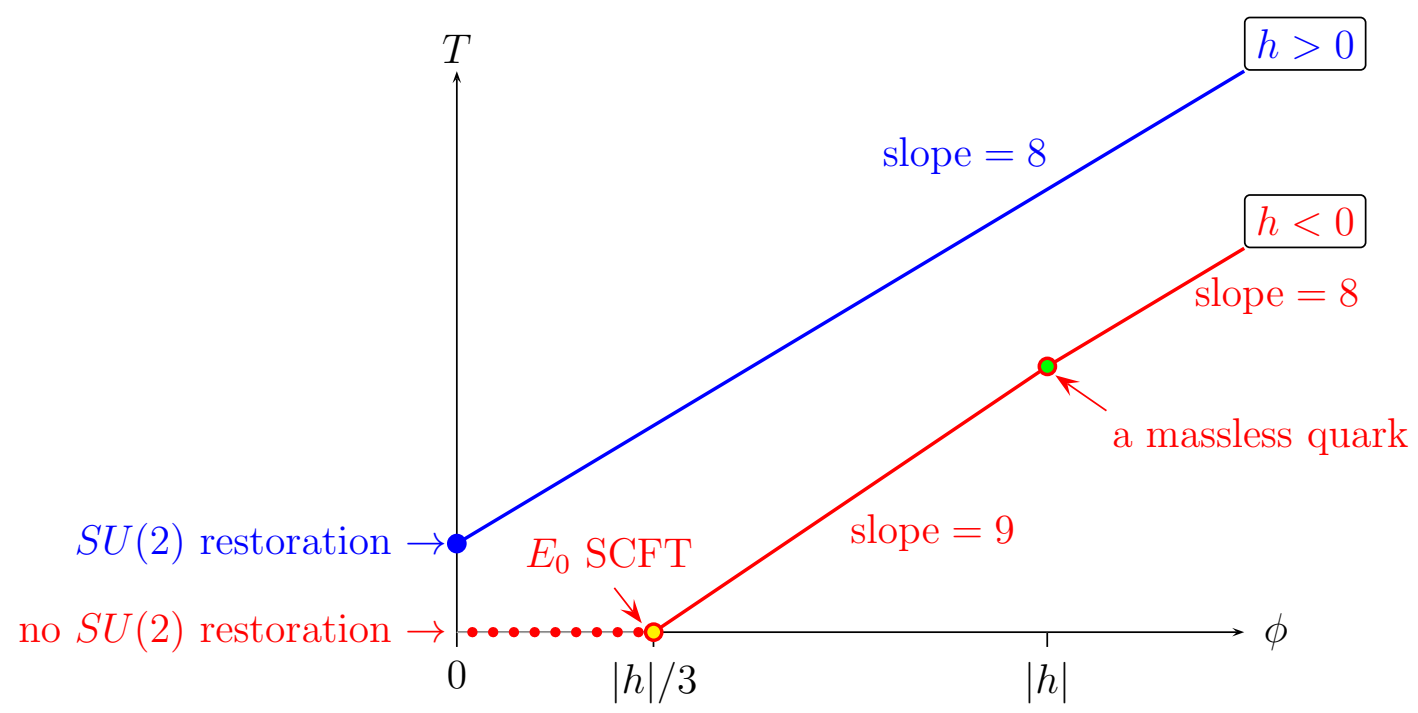

Again, the blue line here plots $T(\phi)$ for a fixed $h>0$ while the red line plots $T(\phi)$ for a fixed $h<0$, but now we have identified all interesting points on both lines. Also, the red line is now dotted left of the $E_{0}$ point $(0 \leq \phi<|h| / 3)$ to indicate that this regime does not really exist in five infinite dimensions but only in the compactified theory. Indeed, in the decompactification limit $L a \rightarrow \infty$, the distance between the $E_{0}$ point and the $\phi=0$ point 
disappears in the field metric

$$
g_{\phi \phi}=\text { via 5D SUSY }=\frac{1}{g_{5 D}^{2}[U(1)]}=\frac{\sqrt{3} / 8 \pi}{L a} \rightarrow 0
$$

and the whole range of $0 \leq \phi \leq(-h / 3)$ becomes invisible.

Such disappearance of 4D phases upon decompactification to 5D is well known in the string theory context. For example, when a type IIA superstring on a Calabi-Yau manifold is promoted to M-theory on the same manifold, the non-geometric phases of the Calabi-Yau disappear from the 5D physics because the moduli space regions where they live collapse to to zero volume in the decompactification limit. The collapse happens due to difference between the $5 \mathrm{D}(\mathcal{N}=1)$ and the $4 \mathrm{D}(\mathcal{N}=2)$ supersymmetric geometries and does not depend on any inherently stringy physics; any other UV completion allowing 4D $\rightarrow$ 5D decompactification of a field theory should behave in a similar way.

And that's precisely what we see in the spectral curve of our deconstructed theory. When compactified on a circle, the theory acquires a "non-geometric" phase occupying the $0 \leq \phi<$ $(-h) / 3$ range of the moduli space, but this phase disappears in the decompactification limit $L a \rightarrow \infty$. Since the deconstruction process requires finite $L$ and hence finite fifth dimension, we have duly deconstructed the non-geometric phase of the compactified theory. But we should not try to interpret this phase in $5 \mathrm{D}$ terms because it's an artefact of compactification.

Let us briefly compare our deconstructed example to a string-theoretical UV completion of the same $5 \mathrm{D}$ theory, i.e., $\tilde{D}_{0}$. Specifically, let us use the type I' superstring (orientifold of the type IIA on a circle) where the $\tilde{D}_{0}$ is realized on a D4-brane probe located near and O8 orientifold plane; the other O8 plane and all 16 D8 branes are far away from the probe. The $5 \mathrm{D}$ scalar field $\phi$ corresponds to the distance between the D4 and the O8; perturbatively, the gauge theory on the probe is enhanced from $U(1)$ to $S U(2)$ for $\phi=0$. The vacuum charge -8 of the orientifold plane creates dilaton gradient in the $\phi$ direction, which makes the gauge coupling on the probe $\phi$ dependent with derivative $\partial T / \partial \phi=+8$. As long as the dilaton's value at the O8 itself is finite, this gives us $T=2 h+8 \phi$ with $h>0$, precisely as for the "ordinary $\mathrm{SYM}_{5}$ " phase of the deconstructed theory, $c f$. the blue line in figure (6.27). 
For $h \rightarrow 0$ the dilaton value at the $\mathrm{O} 8$ blows up and the type I' perturbation theory breaks down, but the S-duality between type I' and heterotic strings allows analytic continuation into the non-perturbative $h<0$ phase. In this phase, the dilaton always blows up at the orientifold plane, but the vacuum charge of the plane changes from -8 to -9 , and an extra D8 brane appears out of the $\mathrm{O} 8$ to balance the charge; the distance between the $\mathrm{O} 8^{-9}$ and the new D8 is proportional to $-h$. Putting a D4 probe right on top of the orientifold in this phase leads to the $E_{0}$ superconformal theory on the probe [2]. And when we move the probe away, we get the Coulomb branch of the $E_{0}$, comprised of one massless vector multiplet with Chern-Simons self-coupling $k=9$, or in terms of the gauge coupling, $T=9 \hat{\phi}$. Note the Chern-Simons level $k=9$ here, it is characteristic of the $E_{0}$ theory.

But besides the vector multiplet living on the D4 probe itself, there is also a quark hypermultiplet due to open strings between the D4 and the D8 brane (which was emitted by the orientifold plane during the phase transition). This quark is generally massive but becomes massless when the probe reaches the D8. And when the D4 probe moves beyond the D8, the quark becomes massive again but its 5D mass flips sign; consequently, the ChernSimons level of the $U(1)$ vector multiplet reduces from +9 to +8 and hence $\partial T / \partial \hat{\phi}=8$ rather than 9. Altogether, we have

$$
T= \begin{cases}9 \hat{\phi} & \text { for } 0 \leq \hat{\phi} \leq m_{e} \\ 8 \hat{\phi}+m_{q} & \text { for } \hat{\phi} \geq m_{q}\end{cases}
$$

Again, this behavior is in perfect agreement with the deconstructed theory with $h<0, c f$. the solid part of the red line in figure (6.27).

Finally, the non-geometric phase of the deconstructed theory - cf. the dotted part of the red line for $0 \leq \phi<(-h / 3)$ - does not have any counterpart in the type I' string theory because we haven't compactified it to $4 \mathrm{D}$. To see this phase in a string implementation of $\tilde{D}_{0}$ we would need a $4 \mathrm{D} \mathcal{N}=2$ construction - for example a D3 probe near a cluster of four $(p, q) 7$-branes in type IIB string, or a type IIA string on a singular Calabi-Yau with a collapsed $d P_{1} 4$-cycle - but this gets us too deep into string theory and away from the main subject of this paper. 
Instead, let us go back to the deconstructed theory and draw its phase diagram in the $(h, \phi)$ parameter space:

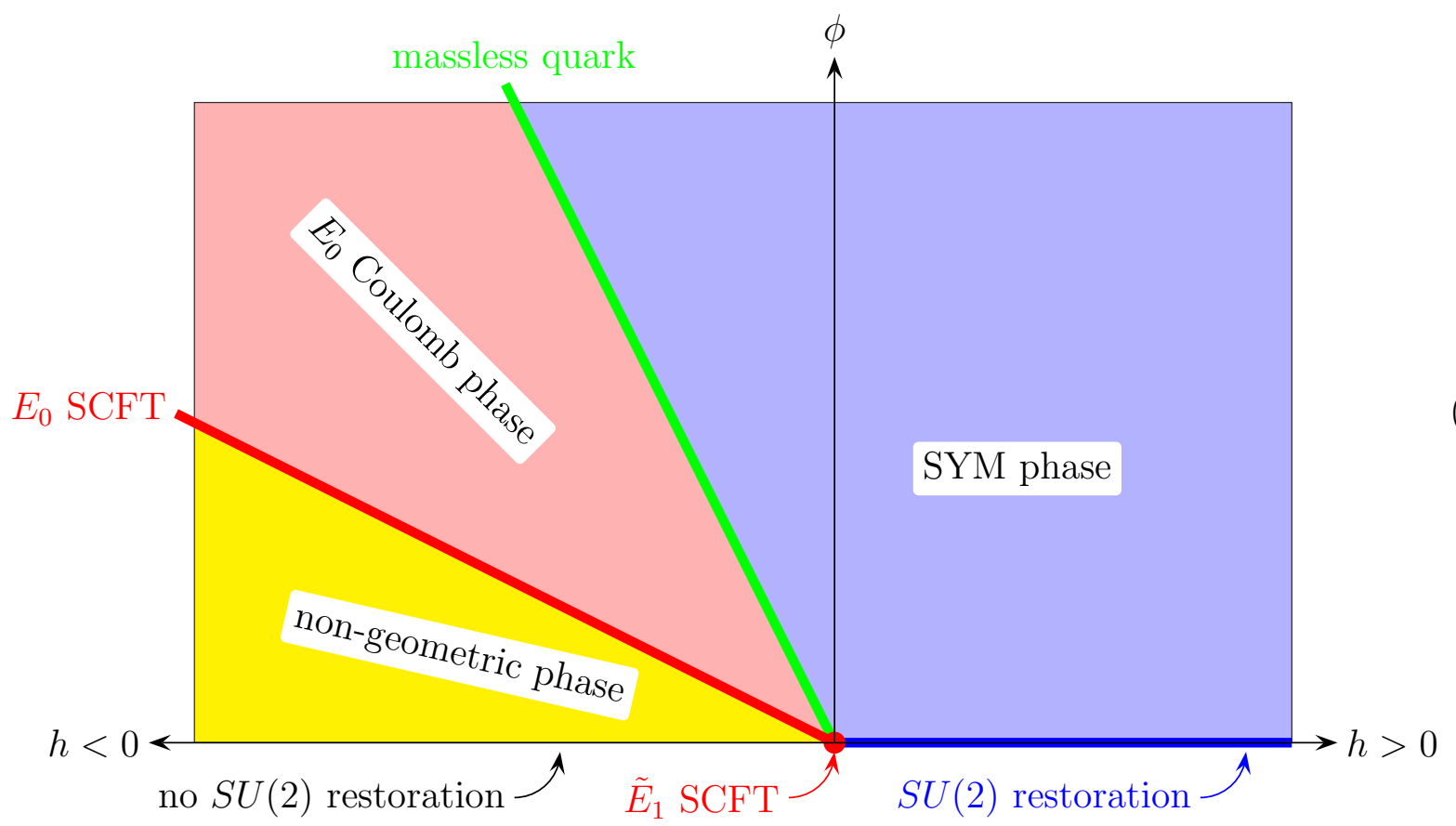

Note that for fixed $\phi>0$ there is no phase transition across the $h=0$ line. In $4 \mathrm{D}$, the branching points of the spectral curve follow the same pattern on both sides of this line cf. eqs. (6.8 and (6.15) - and there are no singularities for $H \rightarrow 0$. Consequently, the 5D physics also continues unperturbed, and the $h>0 \mathrm{SYM}_{5}$ phase continues to negative $h$. Instead, the transition to a new phase — which we identify as a Coulomb branch of an $E_{0}$ theory (with some massive fields added) - happens at $h=-\phi$. Along this transition line, a charged hypermultiplet (a quark) becomes massless while the gauge coupling remains finite. In M-theory terms, such transition is a flop where a 4-cycle changes the sign of its area.

When the parameter $h$ becomes more negative and reaches $h=-3 \phi$, the 5D coupling becomes strong and there is a transition from the Coulomb phase of the $E_{0}$ to the superconformal phase. In M-theory terms, this transition corresponds to 4 -cycle (shaped as a $\mathbb{P}^{2}$ ) collapsing to a point rather than flopping the sign of its area. And left of the transition line $h=-3 \phi$ lies the non-geometric phase, which exist in 4D compactifications of the 5D theory but not in five infinite dimensions. 
The two transition lines intersect at the $(h=0, \phi=0)$ point. The $5 \mathrm{D}$ physics here is superconformal, but the SCFT is $\tilde{E}_{1}$ rather than $E_{0}$. In the type I' string construction of this SCFT point, the dilaton blows up at the $\mathrm{O} 8$ orientifold plane which is just about to emit a D8 brane but has not done it yet, and the D4 probe sits right on top of this strongly-coupled mess. The spectral curve of the $4 \mathrm{D}$ quiver theory is also rather messy at this point, or rather its $O(1 / L a)$ neighborhood in the $(H, \varphi)$ space: $\tau$ is generally strong here, and there are four singular lines with non-commuting monodromies around them. But the general type of singularities agrees with the $\tilde{E}_{1}$ SCFT compactified to 4D [7].

In string theory, the simplest way to produce the phase diagram similar to (6.30) without the non-geometric phase, of course - is via the $(p, q) 5$-brane web construction in type IIB superstring. For the Coulomb branch of the SYM phase, the brane web of the $\tilde{D}_{0}$ model looks like

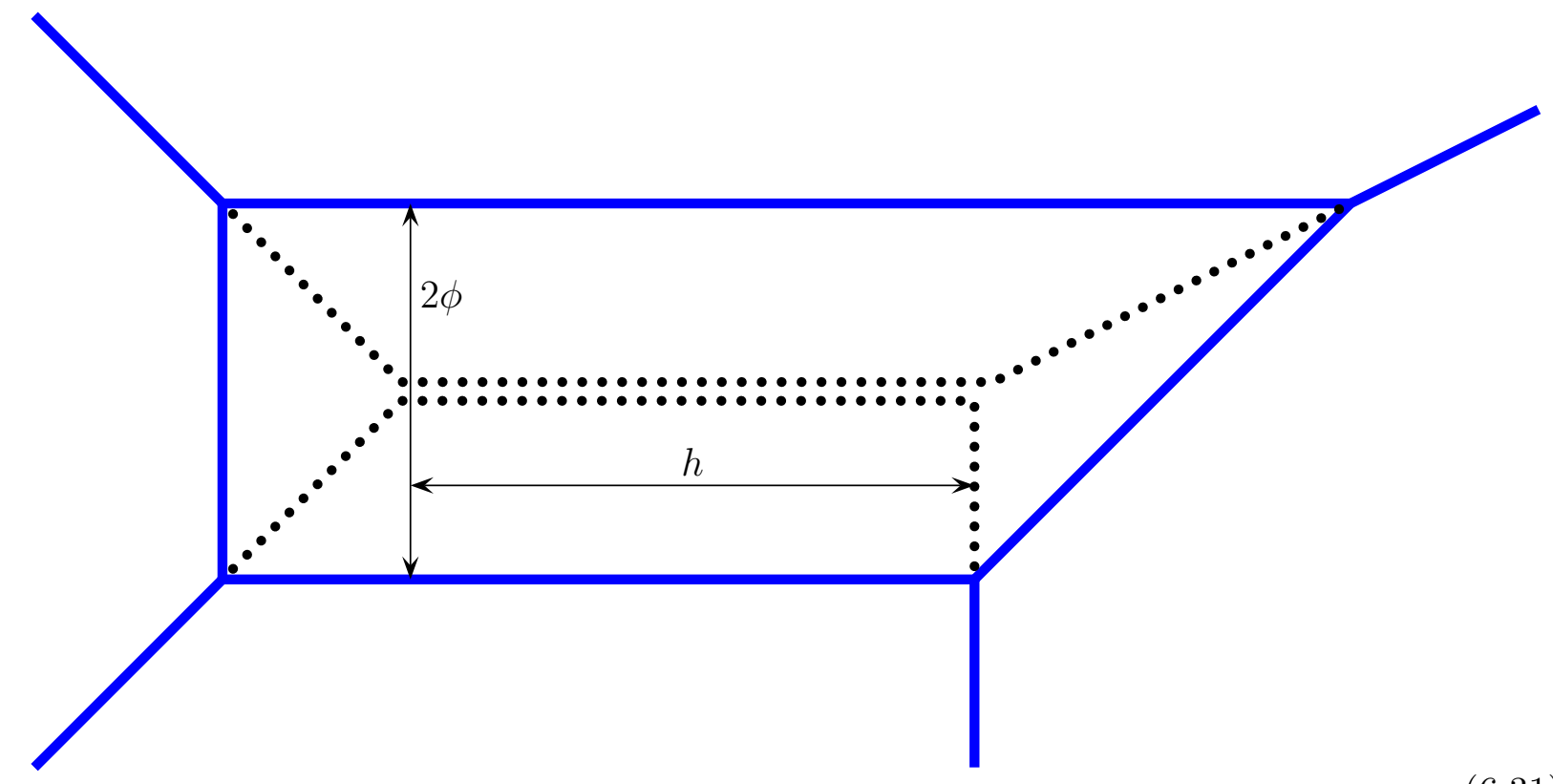

Note that the non-dynamical $h$ parameter here corresponds to relative position of the semiinfinite external lines, while the dynamical modulus $\phi$ controls the internal lines only. For $\phi=$ 0 and $h>0$, two brane segments become coincident (the dotted lines on the diagram (6.31)) over length $h$, and the string connecting these branes produce an $S U(2)$ SYM with 5D gauge coupling $g_{5}^{2} \propto 1 / h$. 
For $h<0$ but $\phi>0$, the web flips between two topologies according to the sign of $\phi+h$ :
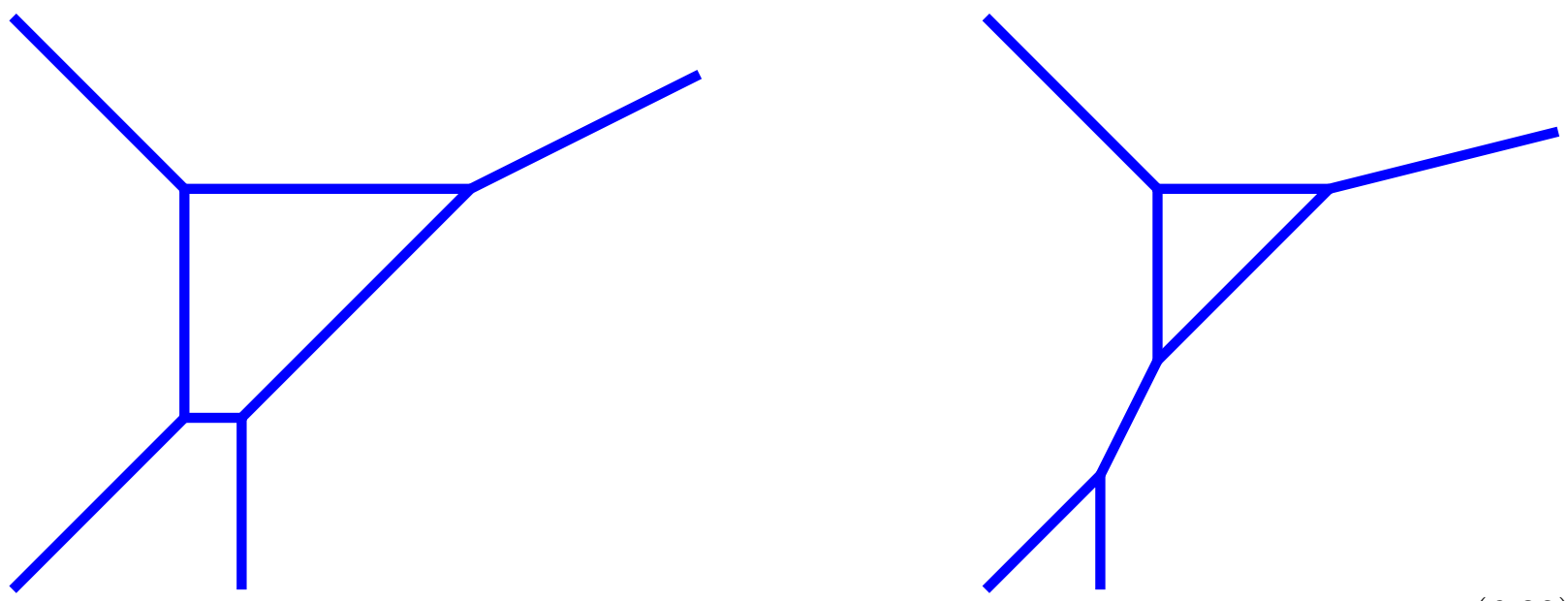

The left web here - for $h<0$ but $h+\phi>0$ - has the same topology as the $h>0$ web (6.32); it corresponds to the extension of the SYM's Coulomb phase from $h>0$ to $-\phi<h<0$. The right web - for $h+\phi<0$ but $h+3 \phi>0$ - has a different topology and describes a different phase of the theory, namely the Coulomb phase of $E_{0}$ (the upper triangle of the web) with an extra massive hypermultiplet (the lower fork). The two webs are related by a segment flop; this is dual to a 4-cycle flop in M-theory. At the flop transition itself (at $h+\phi=0$ ), there is a 4 -brane junction which looks like an intersection of two branes. Here the strings connecting the intersecting branes give rise to a massless charged hypermultiplet:

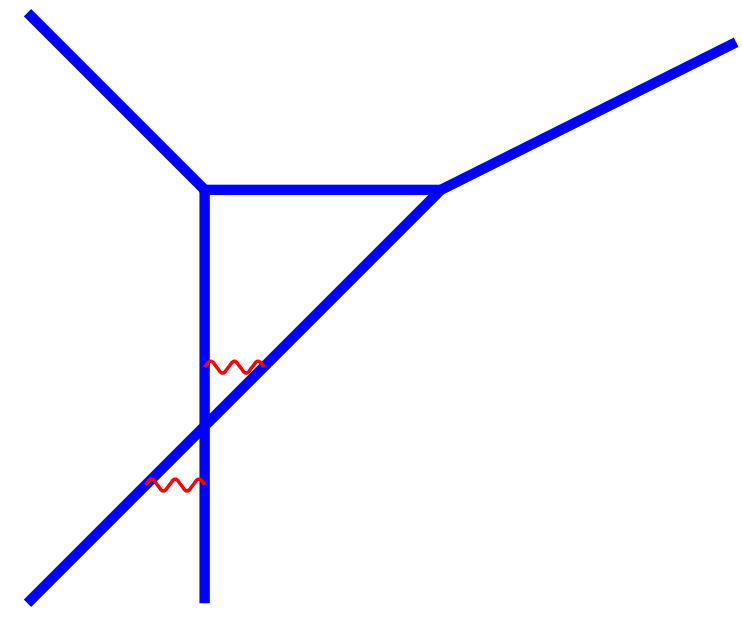

On the other side of the $E_{0}$ Coulomb phase, for $h=-3 \phi$ the triangle collapses to a point. 
In 5D terms, this corresponds to a non-trivial SCFT, in this case $E_{0}$ :

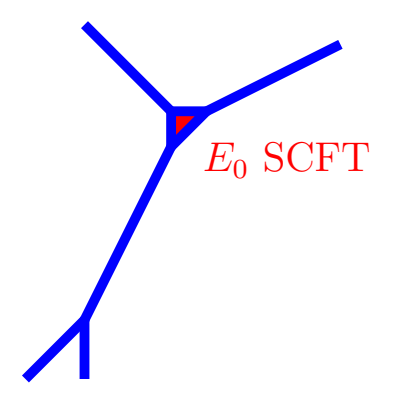

In addition, there is a massive hypermultiplet due to the fork in the lower part of the web.

A different SCFT, namely $\tilde{E}_{1}$ obtains at $h=\phi=0$ when the whole web (except for the external legs) collapses to a point:

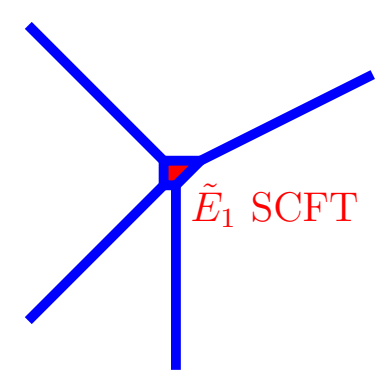

Finally, for $h+3 \phi<0$ the web cannot be build; this impossibility in 5D corresponds to a non-geometric $4 \mathrm{D}$ phase of the deconstructed theory.

\subsection{The $D_{0}$ Model: $n_{c}=2, n_{f}=0, \Delta F=2$.}

Our second model is also an $S U(2)$ SYM in $5 \mathrm{D}$, but with $\theta=0$ instead of $\theta=\pi$. In $4 \mathrm{D}$ quiver terms, this calls for $n_{c}=2, n_{f}=0$, and $\Delta F=2$, hence spectral curve

$$
y^{2}-y \times\left(x^{2}-2 x \cosh (\operatorname{La\varphi })+1\right)+e^{-L a H} \times x^{2}=0 .
$$

The discriminant equation (6.1) for the branching points of this curve factorizes into two quadratic equations

$$
x^{2}-2 \cosh (\operatorname{La\varphi }) \times x+1= \pm 2 e^{-L a H / 2} \times x,
$$


hence

$$
\begin{aligned}
& x_{1,4}=\cosh (\operatorname{La\varphi })+e^{-L a H / 2} \pm \sqrt{\left(\cosh (\operatorname{La\varphi })+e^{-L a H / 2}\right)^{2}-1} \\
& x_{2,3}=\cosh (L a \varphi)-e^{-L a H / 2} \pm \sqrt{\left(\cosh (L a \varphi)-e^{-L a H / 2}\right)^{2}-1}
\end{aligned}
$$

For $h>0$ these branching points lie approximately at

$$
x_{1,2} \approx e^{+L a \varphi} \pm 2 e^{-L a H / 2}, \quad x_{3,4} \approx e^{-L a \varphi} \pm 2 e^{-L a(H+4 \varphi) / 2}
$$

and although this pattern is somewhat different from eq. (6.8) for the previous model, it has a similar crossratio $(6.3) \chi=\frac{1}{16} e^{-L a(H+4 \varphi)}$ and therefore leads to the same gauge coupling

$$
T=2 h+8 \phi
$$

cf. Seiberg formula (6.7). Likewise, there is $S U(2)$ restoration for $\phi \rightarrow 0$ and $h>0$. Indeed, the branching points $(6.38)$ degenerate $\left(x_{1}=x_{4}\right.$ or $\left.x_{2}=x_{3}\right)$ when

$$
\cosh (\operatorname{La\varphi })= \pm 1 \pm e^{-L a H / 2}
$$

and for $h>0$ this happen for

$$
(L a \varphi)^{2} \approx \pm 2 e^{-L a H / 2} \quad \text { or } \quad(L a \varphi-\pi i)^{2} \approx \pm 2 i e^{-L a H / 2}
$$

Clearly this makes two close pairs of Seiberg-Witten points according to eq. (6.11), which indicates $S U(2)$ restoration in $5 \mathrm{D}$.

But despite the similarity between the $\Delta F=1$ and $\Delta F=2$ models for $h>0$, their $h<0$ behaviors are very different. In the present $\Delta F=2$ model, for $\phi>0$, the branching points $x_{1,2,3,4}$ follow the pattern (6.39) as long as $h>-2 \phi$. In $5 \mathrm{D}$ terms, this means that the SYM phase persist to negative $h$ as long as $h>-2 \phi$. And for $h<-2 \phi$ things are getting seriously weird: the branching points asymptote to

$$
x_{1,2} \approx \pm 2 e^{-L a H / 2}, \quad x_{3,4} \approx \mp \frac{1}{2} e^{+L a H / 2}
$$

regardless of $\varphi$, the crossratio becomes $\chi \approx-\frac{1}{4} e^{-L a H} \gg 1$, and all this translates to a finite 
but $\phi$-independent 5D gauge coupling

$$
T=2|h|>0 \text { whenever } h<-2 \phi<0 \text {. }
$$

Finally, for $h<0$ the $S U(2)$ restoration happens not at $\phi=0$ but at $\phi=(-h) / 2$. Indeed, in this region, the degeneration loci (6.41) become

$$
L a \varphi \approx-\frac{1}{2} L a H+\log (2)+\{0 \text { or } \pi i\} \pm e^{+L a H / 2}
$$

which makes two exponentially close pairs (note $e^{+L a H / 2} \ll 1$ ) whose centers differ by a Wilson line $=\pi$; as in eq. (6.11), this is how the $S U(2)$ restoration in $5 \mathrm{D}$ looks to the $4 \mathrm{D}$ spectral curve. On the other hand, the curve does not degenerate for $h<0$ and $\phi \approx 0$, so there is no $S U(2)$ restoration there.

Altogether, we have

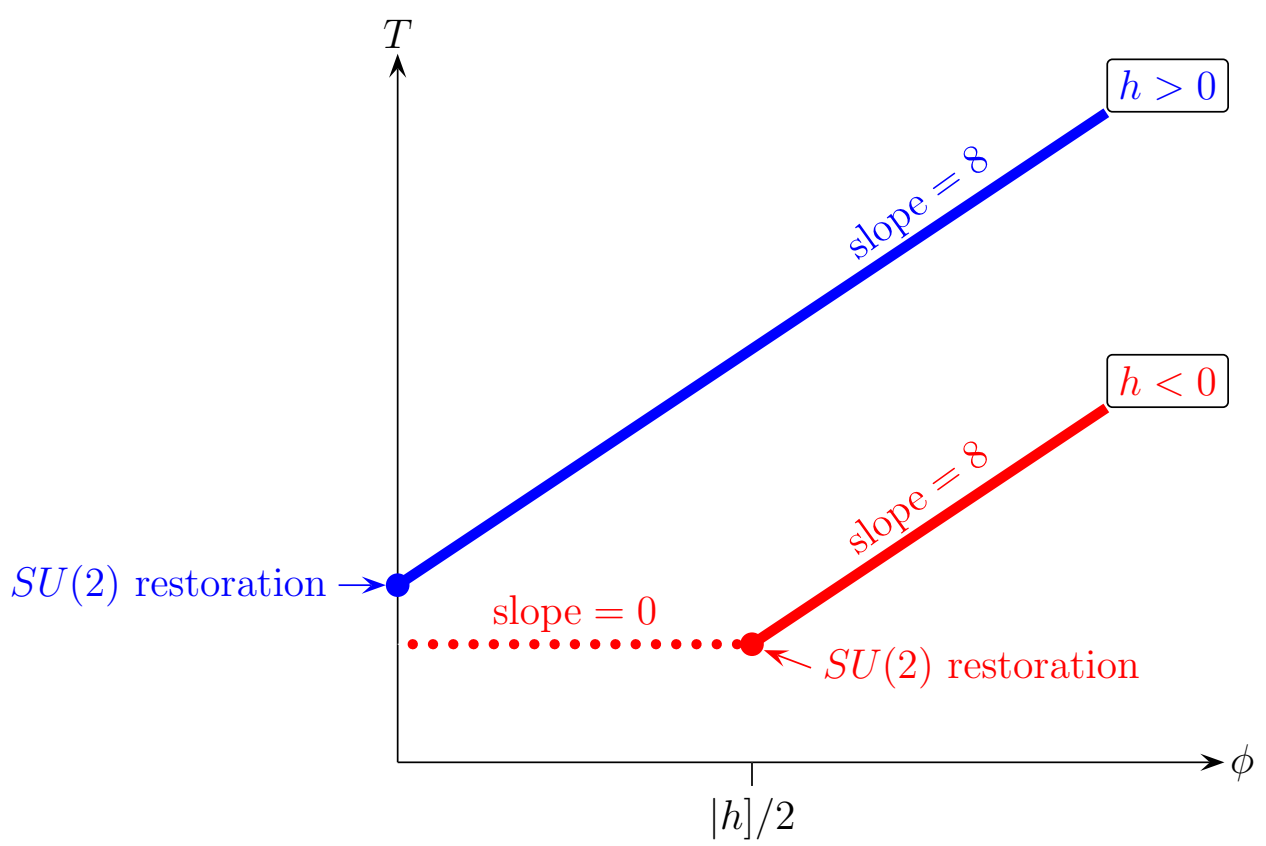

where the dotted red line for $h<0$ and $0<\phi<|h| / 2$ denotes something is wrong in this regime. Indeed, a finite but $\phi$-independent 5D gauge coupling would normally indicate a free $U(1)$ phase, but such a phase cannot possibly connect to an unbroken- $S U(2)$ point at $\phi=|h| / 2$. Instead, an $S U(2)$ point should be the end-point of the 5D moduli space because 
of the $\mathbb{Z}_{2} \subset S U(2)$ reflection of the Coulomb modulus $\hat{\phi} \rightarrow-\hat{\phi}$; in $\phi$ terms, this corresponds to identification

$$
\phi=\frac{-h}{2}+|\hat{\phi}| \quad \Longrightarrow \quad \phi \text { always } \geq \frac{-h}{2}
$$

From the 4D point of view, such premature end of the $\phi$ modulus indicated sudden divergence between the $\varphi$ coordinate of the complex moduli space and between the $\mathcal{N}=2$ superpartner $\mathcal{A}$ of the abelian vector field. Indeed,

$$
\frac{d \mathcal{A}}{d \varphi}=\frac{2 \sinh (L a \varphi)}{2 \pi i} \times \oint \frac{d x}{\sqrt{\left(x-x_{1}\right)\left(x-x_{2}\right)\left(x-x_{3}\right)\left(x-x_{4}\right)}}
$$

where the integration contour is the electric cycle of the spectral curve, i. e. a loop around a branch cut connecting $x_{1}$ with $x_{2}{ }^{\langle 12\rangle}$; the pre-integral factor $2 \sinh (\operatorname{La} \varphi)$ here compensates for the logarithmic definition of the $\varphi$ modulus, $c f$. eq. (4.17). As long as the branching points are as in eq. (6.39), eq. (6.48) evaluates to

$$
\frac{d \mathcal{A}}{d \varphi}=1+O\left(e^{-L a(H+2 \varphi)}\right)
$$

thus in the decompactification limit, $\mathcal{A}=\varphi+$ const with exponentially good accuracy. However, when the branching point pattern changes from (6.39) to (6.43) for $h+2 \phi<0$, eq. (6.48) yields

$$
\frac{d \mathcal{A}}{d \varphi}=\frac{i}{2} e^{+L a(H+2 \varphi) / 2} \ll 1
$$

thus the vector's superpartner $\mathcal{A}$ no longer tracks $\varphi$. Instead, it decouples: as long as $\operatorname{Re} \varphi<(-h / 2)$, the actual value of $\varphi$ does not matter anymore.

Consequently, we would like to map $\varphi$ onto a different holomorphic coordinate which tracks $\varphi$ for $\operatorname{Re} \varphi>(-h / 2)$ but bottoms out at $\operatorname{Re} \varphi=(-h / 2)>0$. From the $\mathcal{N}=2$ point of view it would be best to use the vector's superpartner $\mathcal{A}$ itself, but since it suffers nontrivial monodromies at the Seiberg-Witten points (6.45), we would rather use something simpler. Specifically, we want a holomorphic coordinate $\hat{\varphi}$ which lives on a half-cylinder, i.e. $\hat{\varphi} \equiv \hat{\varphi}+\frac{2 \pi i}{L a}$ and $\hat{\varphi} \equiv-\hat{\varphi}$, and whose real part $\operatorname{Re} \hat{\varphi}$ becomes the 5D modulus $\hat{\phi}(c f$.

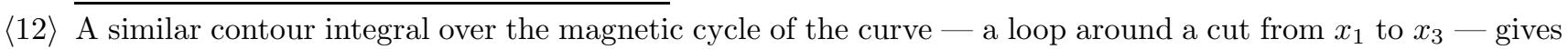
$d \mathcal{A}_{D} / d \cosh (\operatorname{La\varphi })$ where $\mathcal{A}_{D}$ is the superpartner of the magnetic dual of the vector field. 
eq. (6.47)) in the decompactification limit. And since $\varphi$ itself lives on a half-cylinder, the map between $\varphi$ and $\hat{\varphi}$ works according to

$$
\cosh (\operatorname{La\hat {\varphi }})=e^{+L a H / 2} \times \cosh (\operatorname{La\varphi }) .
$$

As promised, for $L a \rightarrow \infty$ eq. (6.51) reduces to $\lfloor\hat{\varphi}\rfloor=\lfloor\varphi\rfloor+\frac{1}{2} H$ and hence eq. (6.47) for the real $5 \mathrm{D}$ variables $\hat{\phi}$ and $\phi$. But near the $S U(2)$ restoration points, the $\hat{\varphi}$ variable becomes double-valued, hence eq. (6.45) becomes

$$
(L a \hat{\varphi})^{2}= \pm 2 e^{+L a H / 2} \quad \text { and } \quad(L a \hat{\varphi}-\pi i)^{2}= \pm 2 e^{+L a H / 2} .
$$

Note Seiberg-Witten's tr((adjoint scalar $\left.)^{2}\right)$ here corresponds to $(L a \hat{\varphi})^{2}$ or $(L a \hat{\varphi}-\pi i)^{2}$, similarly to $(L a \varphi)^{2}$ or $(L a \varphi-\pi i)^{2}$ for the $S U(2)$ restoration at $\phi \rightarrow 0$ for $h>0$, cf. eq. (6.42).

In fact, this symmetry between the two $S U(2)$ restorations at $(\phi=0, h>0)$ and $(\hat{\phi}=$ $0, h<0)$ is an exact symmetry of the spectral curve of the $4 \mathrm{D}$ quiver theory. To make it manifest, we rewrite the spectral curve (6.36) as

$$
\left(z+\frac{1}{z}\right)+e^{-L a H / 2} \times\left(x+\frac{1}{x}-2 \cosh (\operatorname{La\varphi })\right)=0, \quad z=-\frac{y}{x} \times-e^{L a H / 2} .
$$

The coordinates $x$ and $z$ appear here in a similar way, and the curve is symmetric with respect to simultaneous exchanges of

$$
x \leftrightarrow z, \quad H \leftrightarrow-H, \quad \varphi \leftrightarrow \hat{\varphi}
$$

where $\hat{\varphi}$ is exactly as in eq. (6.51).

In light of this symmetry, we deconstruct the 5D phase diagram of our present model — or 
rather its Coulomb branch — as a single phase bounded by two separate $S U(2)$ restorations:

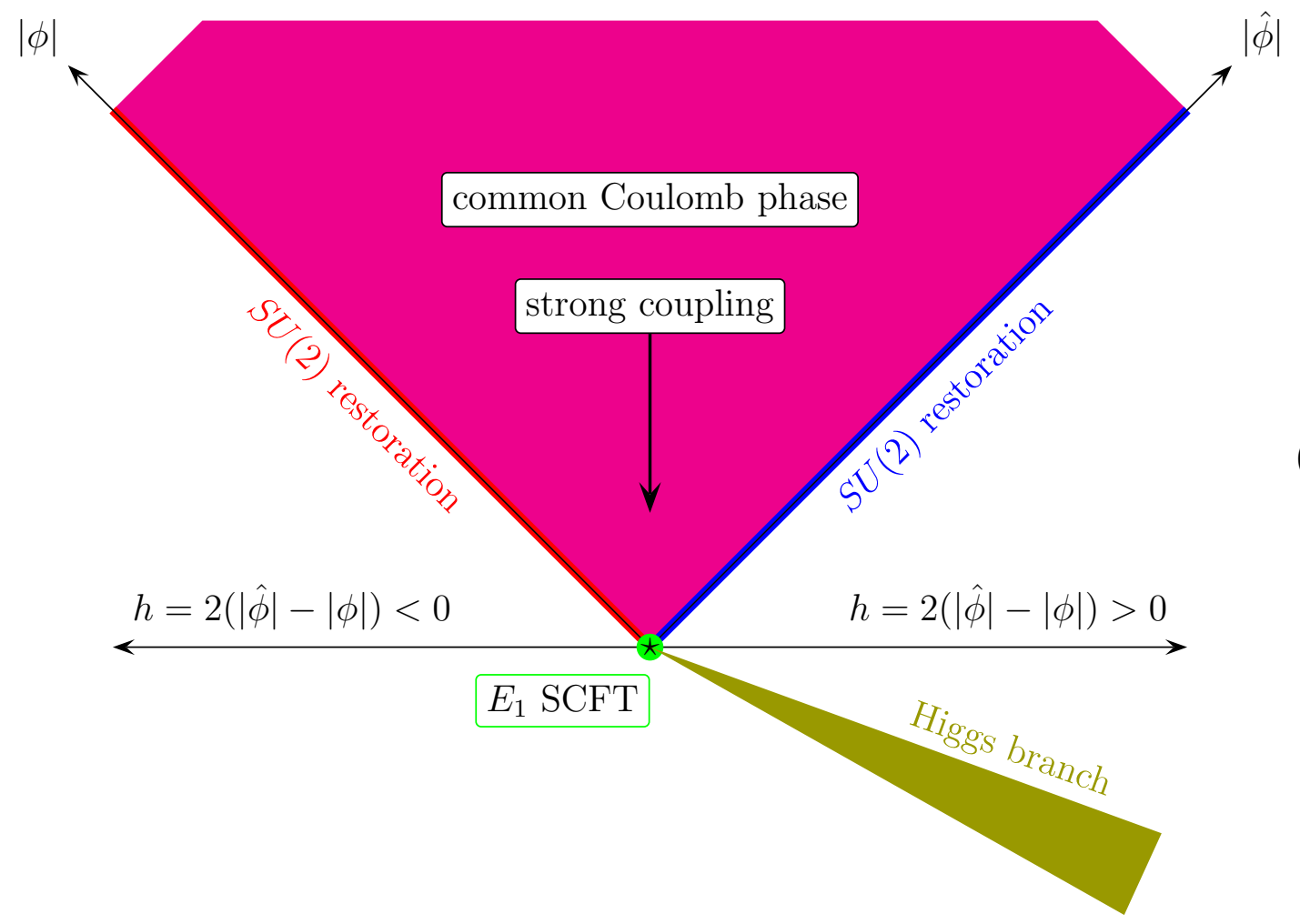

On this diagram, the 5D coupling has a vertical gradient, the higher the weaker, $T \rightarrow \infty$ as one goes up. And in the opposite direction, the coupling becomes infinite $(T=0)$ in the bottom corner $h=\phi=\hat{\phi}=0$, so there instead of a SYM we have a non-trivial superconformal theory. To identify the SCFT in question, we consider the degeneration of the spectral curve in this region and notice that all four degeneration loci (6.41) pass through the $O(1 / L a)$ neighborhood of the $(H=0, \varphi=0)$ point. Moreover, for $H=0$ two of the four singularities collide at $\cosh (\operatorname{La} \varphi)=0$ creating a double singularity. (In Kodaira terms, $I_{1}+I_{1} \rightarrow I_{2}$.) This singularity structure is characteristic of $4 \mathrm{D}$ compactification of the $E_{1}$ SCFT in $5 \mathrm{D}$, and so we identify the bottom corner of diagram (6.55) as the $E_{1}$ point. Also, there is a baryonic branch here corresponding to the Higgs branch of the $E_{1}$. Indeed, at the $I_{2}$ singularity at $H=\cosh (\operatorname{La} \varphi)$ the spectral curve factorizes according to

$$
y^{2}-y \times\left(x^{2}+1\right)+x^{2}=\left(y-x^{2}\right) \times(y-1)=0,
$$

and we saw in $\S 5$ that such factorization indicates a baryonic branch with two $\mu=0$ flavors. 
Ideally, to prove the 5D SCFT at $h=\phi=\hat{\phi}=0$ is indeed the $E_{1}$, we would like to see its enhanced "flavor" symmetry $E_{1}=S U(2)$. Unfortunately, this symmetry does not show up in 4D - presumably, its broken by the deconstruction - and instead, we have to rely on less transparent signatures such as singularities of the spectral curve in 4D and the Higgs branches.

We conclude this section by comparing the phase diagram (6.55) of the deconstructed $n_{c}=2, n_{f}=0, \theta=0$ model with a stringy implementation of the same 5D theory, i. e. $D_{0}$. Again, we use the type IIB 5-brane web construction. On the Coulomb branch of $D_{0}$, regardless of $h>0$ or $h<0$, the web contains a rectangular box:
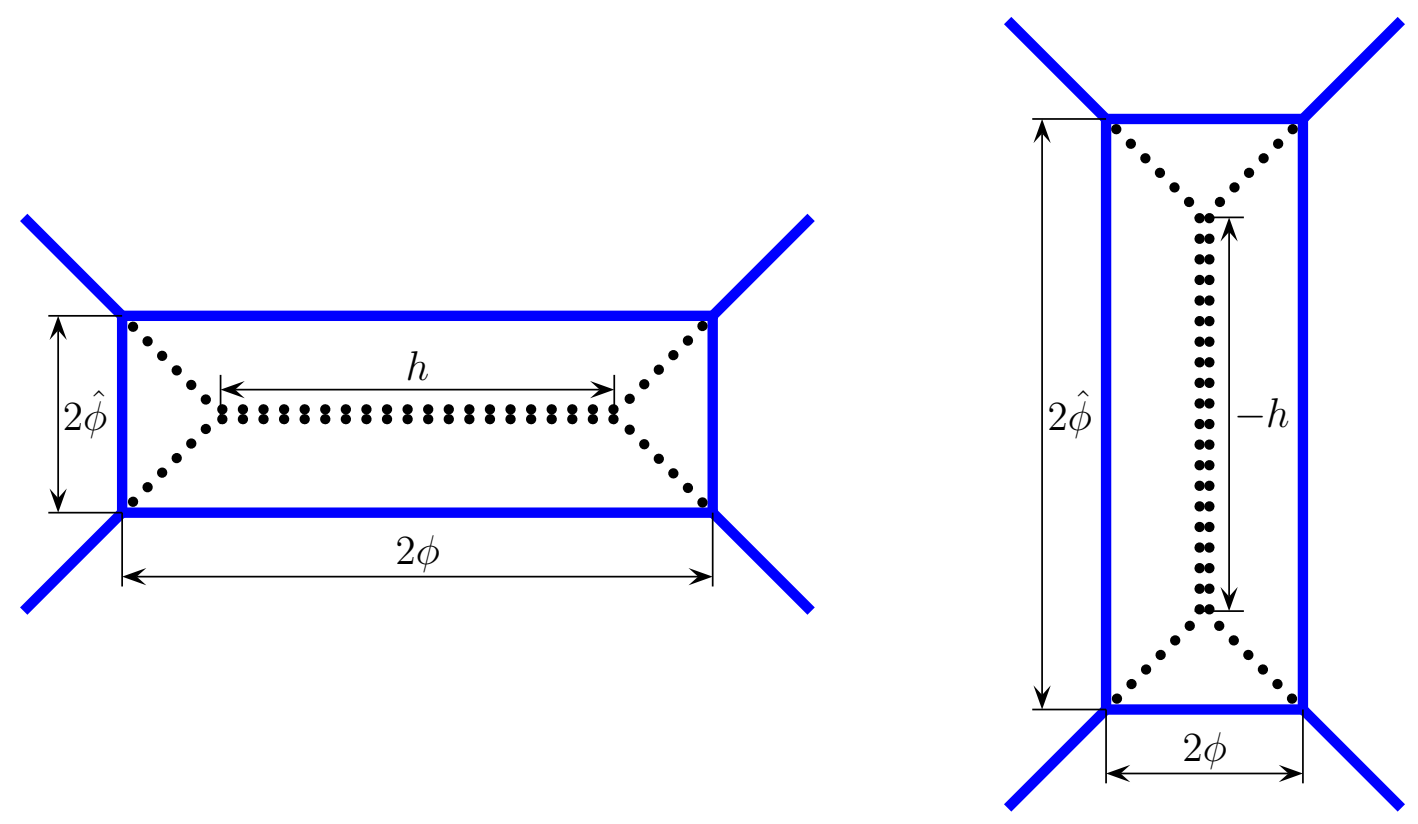

The left web here is for $h>0$ and the right web for $h<0$, and the only difference between them is which side of the box is longer; the topology is the same, and there is no flop transition. For $\phi \rightarrow 0$ or $\hat{\phi} \rightarrow 0$ - whichever happens first - the box collapses to a pair of coincident line segments (the dotted lines on the diagram (6.57), either web); the strings between those coincident branes give rise to an $S U(2)$ SYM with 5D gauge coupling $g_{5}^{2} \propto 1 /|h|$ 
For $h=0$ and $\phi=\hat{\phi} \rightarrow 0$, the box collapses to a point

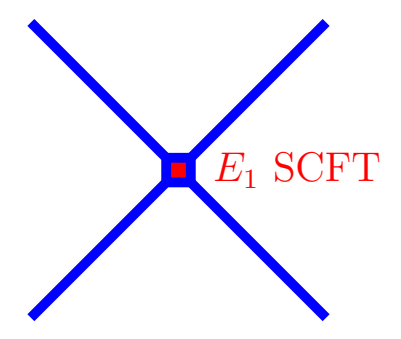

giving rise to the $E_{1}$ superconformal theory in $5 \mathrm{D}$. This theory has an $S U(2)=E_{1}$ global "flavor" symmetry, but it isn't manifest in the brane-web picture. Likewise, the deconstructed theory does not have an enhanced flavor symmetry at $H=0$ at the $4 \mathrm{D}$ quiver level. Presumably, in 5D the enhanced symmetry is limited to the marginal operators of the SCFT, but the irrelevant operators stemming from a UV completion - deconstructive or stringy — break the symmetry.

Finally, the $E_{1}$ web (6.58) can be reconnected as two intersecting whole branes (infinite in all directions), and then the two branes can move away from each other (in a direction perpendicular to both):

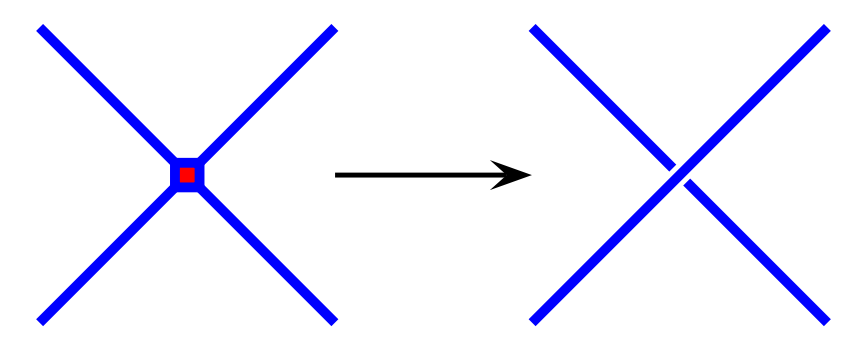

This is the Higgs branch which connects to the Coulomb branch at the $E_{1}$ point; it corresponds to the exotic baryonic branch of the quiver theory.

To summarize, we have seen that the deconstructed $D_{0}$ theory has exactly the same phase structure as the $D_{0}$ completed via string theory. 


\subsection{Models With Flavor: $n_{c}=n_{f}=2$.}

In this section we explore two models with $n_{f}=2$, one model with $\Delta F=1$ and the other with $\Delta F=0$. Each model has three non-dynamical parameters, namely $h, m_{1}$, and $m_{2}$, but for simplicity we restrict our analysis to $\left|m_{1}\right|=\left|m_{2}\right|$ where in $5 \mathrm{D}$ we expect $U(2)$ flavor symmetry for $m \neq 0$ and $S O(4)$ for $m=0$.

We begin with the $\Delta F=1$ model with $m_{2}=-m_{1}=m \geq 0$, or in $4 \mathrm{D}$ terms $\mu_{1,2,3}=$ $\left(V e^{-a M}, V e^{+a M}, 0\right)$ where $\operatorname{Re}(M)=m$, hence the spectral curve

$$
y^{2}-y \times\left(x^{2}-2 \cosh (\operatorname{La\varphi }) \times x+1\right)-e^{-L a H} \times x \times\left(x^{2}-2 \cosh (\operatorname{LaM}) \times x+1\right)=0 .
$$

Note that for $M \neq 0$, the flavor symmetry of the quiver theory itself is $U(1)^{2}$ rather than $U(2)$, but the discrete $\mathbf{C}$ symmetry (4.25-27) of the spectral curve permutes the two 5D flavors and hence acts as a custodial symmetry: it assures that the low-derivative operators in 5D are $U(2)_{F}$ invariant, although the higher-derivative operators do not have this symmetry. And for $M=0$ (or $M=\frac{\pi i}{L a}$ ) the quiver has flavor symmetry $U(2)$, but $\mathbf{C}$ acts as a custodial symmetry of the $U(2) \rightarrow S O(4)$ symmetry enhancement in the $5 \mathrm{D}$ continuum limit.

Along the Coulomb branch, the spectral curve (6.60) generally has four branching points at

$$
x_{1,4}=a \pm \sqrt{a^{2}-1}, \quad x_{2,3}=b \pm \sqrt{b^{2}-1}
$$

where

$$
a, b=\cosh (L a \varphi)-e^{-L a H} \pm e^{-L a H / 2} \times \sqrt{2 \cosh (\operatorname{LaM})+e^{-L a H}-2 \cosh (\operatorname{La\varphi })} .
$$

The curve has four simple singularities ( $I_{1}$ in Kodaira terms) at

$$
\sinh ^{2} \frac{L a \varphi}{2}= \pm e^{-L a H / 2} \sinh \frac{L a M}{2} \quad \text { and } \quad \cosh ^{2} \frac{L a \varphi}{2}= \pm e^{-L a H / 2} \cosh \frac{L a M}{2}
$$

where two of branching points coincide. For

$$
\cosh (L a \varphi)=e^{-L a H}=e^{\mp L a M}
$$

two of the singularities (6.63) merge into a double singularity, $I_{1}+I_{1} \rightarrow I_{2}$. In addition, 
there is another double singularity at

$$
2 \cosh (L a \varphi)=2 \cosh (L a M)+e^{-L a H} .
$$

At all these double singularities the spectral curve factorizes:

$$
\begin{aligned}
& \text { for }(6.65), \quad\left(y-\left(x-e^{-L a M}\right)\left(x-e^{+L a M}\right)\right) \times\left(y+e^{-L a H} x\right)=0, \\
& \text { for }(6.64), \quad\left(y-x\left(x-e^{\mp L a M}\right)\right) \times\left(y+\left(e^{\mp L a M} x-1\right)\right)=0 .
\end{aligned}
$$

According to $\S 5$, this indicates baryonic branches connected to the Coulomb branch at these points, namely the ordinary baryonic branch $B_{12}$ at (6.65), and exotic baryonic branches $B_{13}$ and $B_{23}$ at (6.64). (The subscripts of $B$ here refer to the flavors of the baryonic VEV.) In the decompactification limit $L a \rightarrow \infty$, the locations of these baryonic branches become

$$
\begin{aligned}
& B_{12} \text { branch }: \quad \phi=\max (m,-h), \\
& B_{13} \text { branch }: \quad \phi=0, \quad h=m, \\
& B_{23} \text { branch }: \quad \phi=m, \quad h=-m .
\end{aligned}
$$

Finally, for $M=0$ or $\frac{\pi i}{L a}$, another pair of $I_{1}$ singularities (6.63) merges into an $I_{2}$ at $\varphi=M$, but this time the spectral curve does not factorize all the way; instead, it has one branch cut and one pole (same on both sheets). Consequently, at this point we have a mesonic branch. Note that for $h>0$ this branch is located very close to the $B_{12}$ baryonic branch, and in the 5D limit the two Higgs branches are rooted at the same place $m=\phi=0$. And indeed, in classical $\mathrm{SQCD}_{5}$ with $n_{c}=n_{f}=2$, the mesons and the baryons are related by the $O(4)$ flavor symmetry; although in the quantum theory only $S O(4) \subset O(4)$ is a true symmetry while the discrete $\mathbb{Z}_{2}=O(4) / S O(4)$ 'isoparity' is anomalous, the anomaly does not affect the moduli space for $h>0$. In $4 \mathrm{D}$ however, the anomaly is more powerful, and the instanton effects separate the mesonic and the baryonic branches by $\Delta(\operatorname{La\varphi })=O\left(e^{-L a H}\right)$. In quiver terms, the instantons here are diagonal, i.e, one instanton in each $S U(2)_{\ell}$ factor of the $[S U(2)]^{L}$ gauge group, and that's why the effect is so small for $h>0$.

And now consider the Coulomb branch of the quiver. In the decompactification limit $L a \rightarrow \infty$, the branching points (6.61) of the spectral curve form several distinct patterns 
depending on $\phi, h$ and $m$; as usual, this leads to different phases in 5D. Let us start with the phase structure for $m=0$ :

$\star$ For $h>0$, there is only one phase: for any $\phi>0$, the branching points are

$$
x_{1,2}=e^{L a \varphi} \pm 2 i e^{L a(\varphi-H) / 2}, \quad x_{3,4}=e^{-L a \varphi} \pm 2 i e^{L a(-3 \varphi-H) / 2},
$$

their crossratio is $\chi=\frac{-1}{16} e^{L a(H+3 \varphi)}$, and hence in $5 \mathrm{D}$

$$
T=2 h+6 \phi
$$

in perfect agreement with the Seiberg formula (6.7) for $n_{f}=2$. For $\phi \rightarrow 0$, the situation is more complicated and the spectral curve develops multiple singularities near $L a \varphi=0, \pi i$. Specifically, there is one Seiberg-Witten pair of simple $\left(I_{1}\right)$ singularities at

$$
(L a \varphi-\pi i)^{2} \approx \pm 2 e^{-L a H}
$$

and another pair of double $\left(I_{2}\right)$ singularities at

$$
L a \varphi=0, e^{-2 L H}
$$

The latter pair is also of Seiberg-Witten type but correspond to $\mathcal{N}=2 S U(2)$ gauge theory with two massless flavors rather than flavorless SYM as in eq. (6.72). ${ }^{\langle 13\rangle}$ Thus, above the Seiberg-Witten scale (which is exponentially small for $L a \rightarrow \infty$ ) we have $S U(2)$ restoration for $L a \varphi=0$ and $\pi i$, and for $L a \varphi=0$ we also have two massless quarks. In $5 \mathrm{D}$ terms, this means that for $\phi=0$ we have unbroken $S U(2)$ gauge theory with two massless flavors; in string constructions, this $5 \mathrm{D}$ theory is known as the $D_{2}$ after its global symmetry.

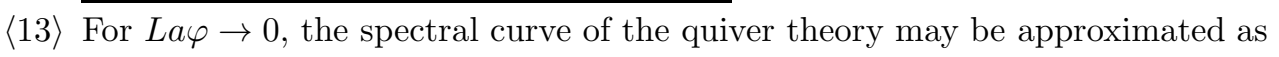

$$
y^{\prime 2}=\left(x^{\prime 2}-(L a \varphi)^{2}\right)^{2}-4 e^{-L a H} \times x^{2}
$$

where $x^{\prime}=x-\cosh (\operatorname{La\varphi }) \approx x-1$ and $y^{\prime}=2 y-\left(x-e^{\operatorname{La} \varphi}\right)\left(x-e^{-L a \varphi}\right)$. This curve looks exactly like the $\mathcal{N}=2$ Seiberg-Witten curve of $S U(2)$ gauge theory with two massless flavors, where the role of $\operatorname{tr}\left((\text { adjoint scalar })^{2}\right)$ is played by $(L a \varphi)^{2}$ and the role of $\Lambda_{\mathrm{SW}}^{2}$ by $e^{-L a H}$. 
* For $h=0$, the branching points of the spectral curve are as in eq. (6.70) as long as $\phi>0$, and hence the 5D coupling is as in eq. (6.71). However, for $\phi \rightarrow 0$ we now have $T=0$, meaning infinitely strong $g_{5}$ and hence a non-trivial superconformal theory in 5D. Since this SCFT obtains in the $h=0$ limit of the $D_{2}$ theory we expect it to be $E_{3}$. Unfortunately, we cannot directly verify the $E_{3}=S U(3) \times S U(2)$ global symmetry of the SCFT because it applies only to the marginal and relevant operators of the 5D theory, so instead we look at the singularities of the spectral curve. The curve of $5 \mathrm{D} E_{3}$ SCFT compactified to $4 \mathrm{D}$ should have three singularities, of respective Kodaira types $I_{3}, I_{2}$, and $I_{1}$, and indeed the quiver's curve (6.60) has such singularities for $H=M=0$ : an $I_{3}$ at $\cosh (\operatorname{La\varphi })=+1$, an $I_{2}$ at $\cosh (\operatorname{La} \varphi)=\frac{3}{2}$, and an $I_{1}$ at $\cosh (\operatorname{La\varphi })=-3$, cf. eqs. (6.63) and (6.65).

* Finally, for $h<0$, there are three distinct patterns of the branching points depending on $\phi$ : for $\phi>-h$, the branching points are as in eq. (6.70) and $T=2 h+6 \phi$; for $\frac{-h}{2}<\phi<-h$, we have

$$
x_{1} \approx-4 e^{-L a H}, \quad x_{2} \approx-\frac{1}{4} e^{L a(H+2 \varphi)}, \quad x_{3} \approx-4 e^{-L a(H+2 \varphi)}, \quad x_{4} \approx-\frac{1}{4} e^{+L a H}
$$

with crossratio $\chi=\frac{1}{16} e^{L a(2 H+4 \varphi)}$ and hence $T=4 h+8 \phi$; and for $0<\phi<\frac{-h}{2}$ the branching points are

$$
x_{1} \approx-4 e^{-L a H}, \quad x_{2,3} \approx+1 \pm \frac{1}{2} e^{L a(H+2 \varphi) / 2}, \quad x_{4} \approx-\frac{1}{4} e^{+L a H}
$$

with crossratio $\chi=e^{+L a(H+2 \varphi) / 2} \ll 1$ and hence $T=-h-2 \phi$. Altogether,

$$
\text { for } h<0, \quad T(\phi)= \begin{cases}2 h+6 \phi & \text { for } \phi>-h \\ 4 h+8 \phi & \text { for } \frac{-h}{2}<\phi<-h \\ -h-2 \phi & \text { for } \phi<\frac{-h}{2}\end{cases}
$$


The following diagram summarizes the various regimes of the $D_{2}$ theory for $m=0$ :

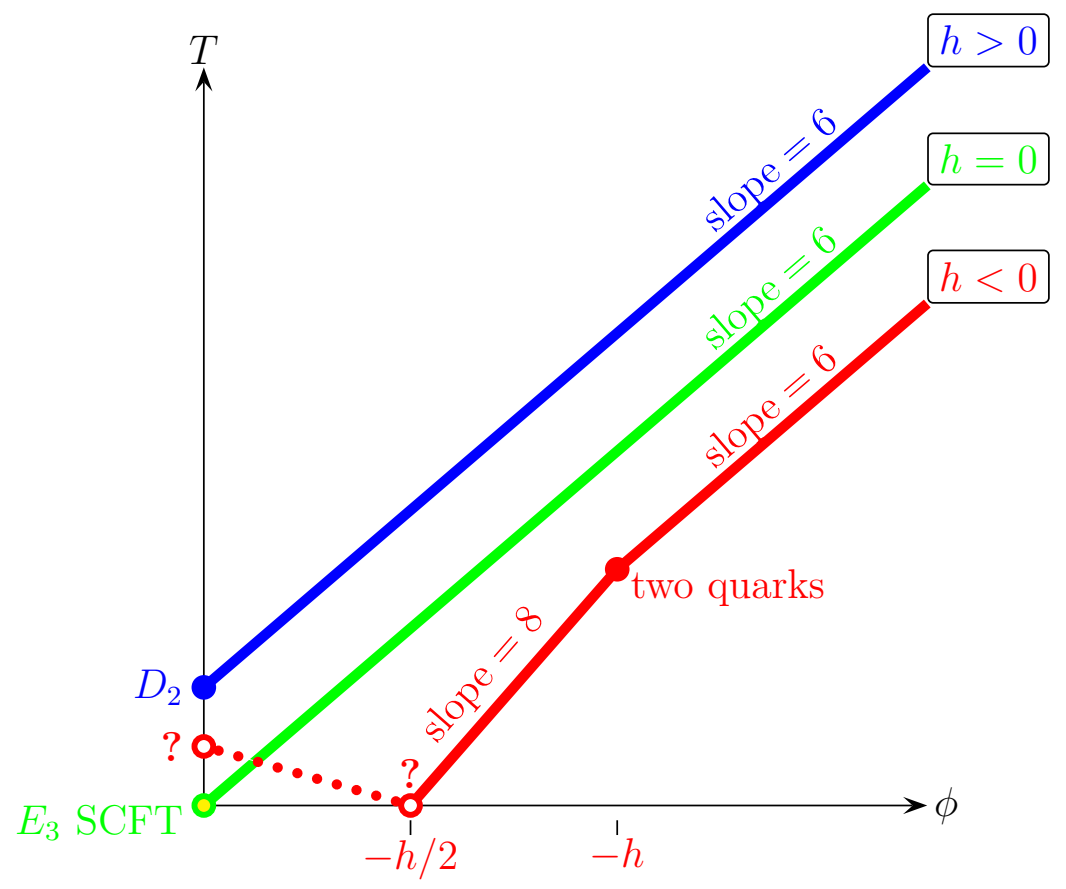

The solid circle on the red line here (for $h<0$ ) indicate a double flop transition at $\phi=-h$. Two quarks become massless at this point and that's where the $B_{12}$ baryonic branch lives for $h<0$. Indeed according to eq. (6.67), the ordinary baryonic branch moves from its classically expected location at $\phi=0$ (for $m=0$ ) to $\phi=-h$.

The physical meaning of the open red circles at $\phi=\frac{-h}{2}$ and $\phi=0$ is moot because the whole dotted segment of the red line is unphysical. From the $4 \mathrm{D}, \mathcal{N}=2$ point of view, for $0 \leq \phi<\frac{-h}{2}$ the scalar superpartner $\mathcal{A}$ of the massless abelian vector decouples from the $\varphi$ modulus. Indeed, for the $x_{1,2,3,4}$ branching points as in eq. (6.76), eq. (6.48) yields

$$
\frac{d \mathcal{A}}{d \varphi} \approx \frac{-L a(H+2 \varphi)}{2 \pi} \times e^{+L a(H+2 \varphi) / 2} \ll 1
$$

To eliminate this unphysical range we change variables from $\varphi$ to $\hat{\varphi}$ according to eq. (6.51). In terms of the $\hat{\varphi}$, its real part $\hat{\phi}$ is non-negative, and in the $\hat{\phi} \rightarrow 0$ limit we have $T=0$ and hence a non-trivial SCFT in 5D. Also in terms of $\hat{\varphi}$, the $O(1 / L a)$ neighborhood of $\hat{\varphi}=0$ contains three singularities (6.63) of the spectral curve, namely an $I_{2}$ singularity at $\cosh (\operatorname{La} \hat{\varphi}) \approx 0$ (note $M=0$ ), and a pair of $I_{1}$ singularities at $\cosh (\operatorname{La} \hat{\varphi}) \approx \pm 2$. This singularity structure in $4 \mathrm{D}$ indicates the $5 \mathrm{D}$ SCFT at $\hat{\phi}=0$ is $E_{1}$. And to confirm this 
identification, we note that there is a Higgs branch growing out of the superconformal point $\hat{\phi}=0$, namely the mesonic branch of the quiver theory.

Altogether, we deconstruct the following Coulomb phase diagram of the $S U(2)$ theory with $n_{f}=2, \Delta F=1$, and $m_{1}=m_{2}=0$ :

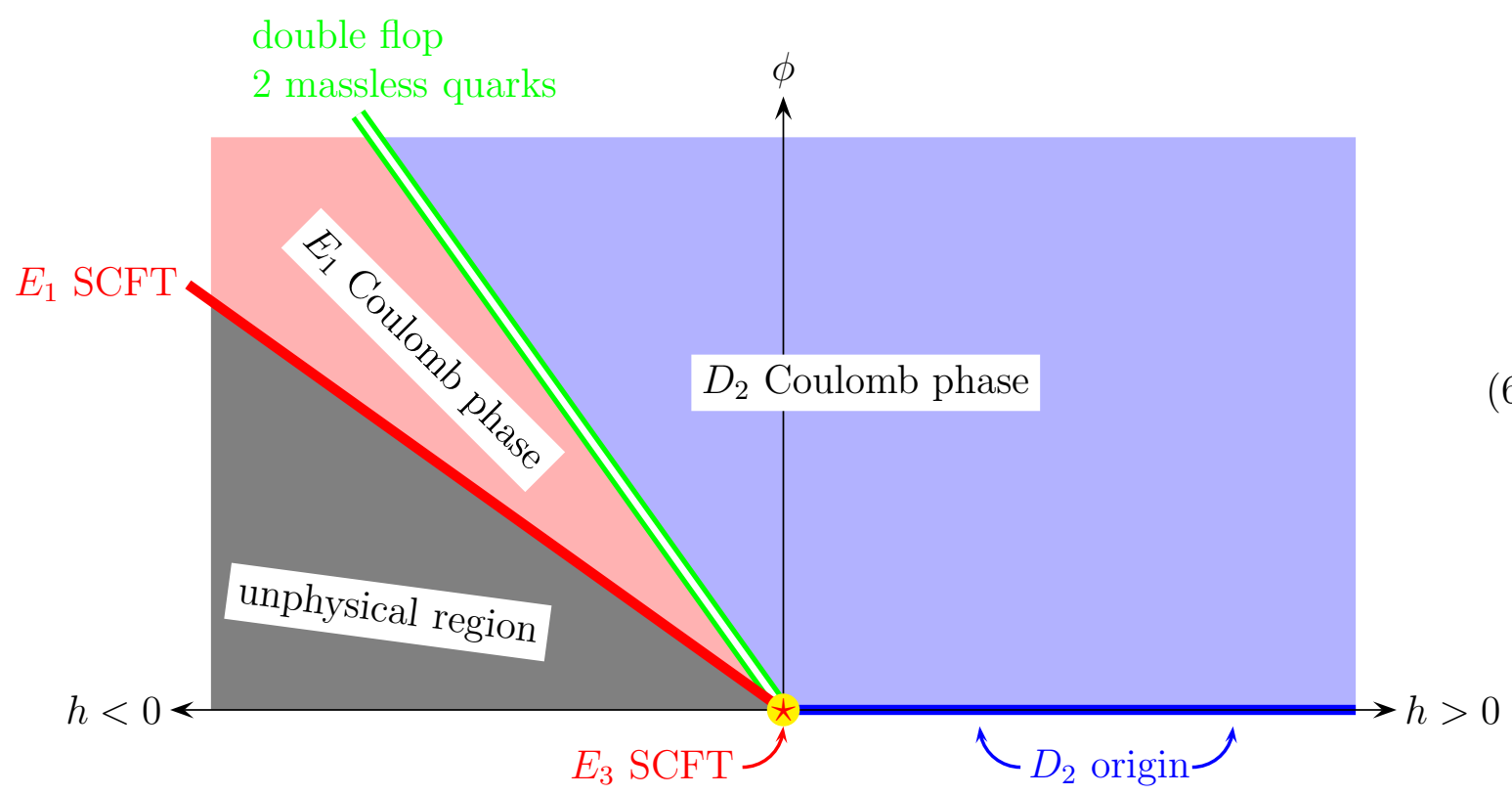

As to the Higgs branches, they are rooted along the $D_{2}$ origin, $E_{1}$ SCFT, and double flop lines according to

$B_{12}$ baryonic

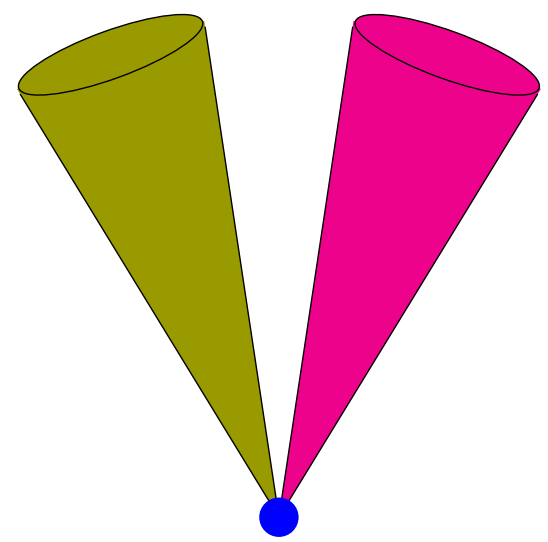

$D_{2}$ origin

$h>0, \phi=0$ mesonic

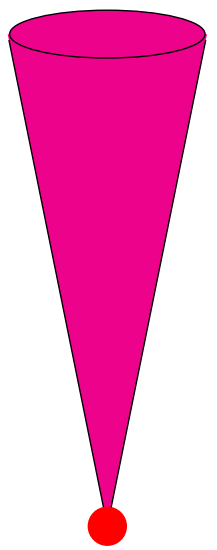

$E_{1} \mathrm{SCFT}$

$h<0, \phi=-h$
$B_{12}$ baryonic

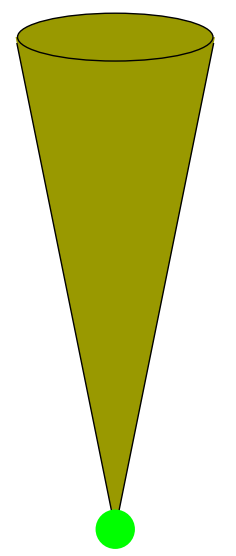

double flop

$h<0, \phi=-h / 2$ 
and also at the $E_{3}$ SCFT point according to

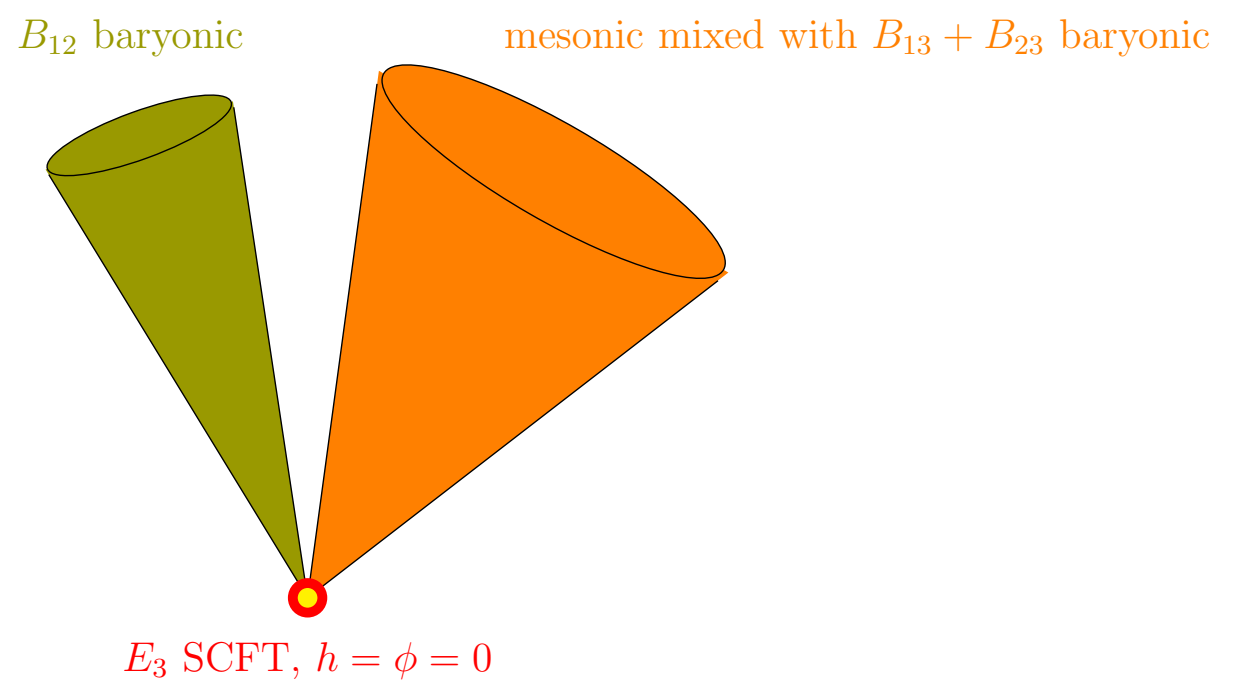

The mixed branch at the $E_{3}$ point has hypermultiplet dimension $=2($ real dimension $=8)$; all other Higgs branches have hypermultiplet dimension $=1($ real dimension $=4)$.

And now consider the quiver theory for $m>0$. Again, we look at patterns of the branching points (6.61) for different values of $\phi$ and $h$.

- For $h>m$ there are two patterns: for $\phi>m$ the roots are as in eq. (6.70) and hence $T=2 h+6 \phi$, while for $0<\phi<m$

$$
x_{1,2}=e^{L a \varphi} \pm 2 e^{L a(H-M) / 2}, \quad x_{3,4}=e^{-L a \varphi} \pm 2 e^{L a(H-M-4 \varphi) / 2},
$$

the crossratio is $\chi=\frac{1}{16} e^{L a(4 \varphi+H-M)}$, and $T=2 h-2 m+8 \phi$. Altogether,

$$
T= \begin{cases}2 h+6 \phi & \text { for } \phi>m \\ 2 h-2 m+8 \phi & \text { for } \phi<m\end{cases}
$$

in perfect agreement with the Seiberg formula for $5 \mathrm{D} S U(2)$ with massive flavors

$$
T=2 h+8 \phi-\sum_{f} \max \left(\phi,\left|m_{f}\right|\right) .
$$

At $\phi=m$, there is a double flop due to two quark flavors becoming massless at the 
same time - note $\phi_{1}=m_{1}=-m$ and $\phi_{2}=m_{2}=+m$. This point on the Coulomb branch is the origin of the ordinary baryonic branch $B_{12}, c f$. eq. (6.67).

Finally, for $\phi=0$ we have $S U(2)$ restoration in 5D. Indeed the singularities (6.63) of the spectral curve form two Seiberg-Witten pairs

$$
(L a \varphi)^{2}= \pm 2 e^{-L a(H-M) / 2} \quad \text { and } \quad(L a \varphi-\pi i)^{2}= \pm 2 e^{-L a(H-M) / 2}
$$

separated by Wilson line $=\pi$. Note $\Lambda_{\mathrm{SW}}^{2}=e^{-L a(H-M) / 2}$ here indicates classical 4D $S U(2)$ coupling $\frac{8 \pi^{2}}{g_{4}^{2}}=L a(h-m)$ and hence $5 \mathrm{D}$ coupling $T=h-m$. This agrees with the $\phi \rightarrow 0$ limit of the abelian coupling: according to eq. (6.84), T( $\phi=0)=2 \times(h-m)$; the factor of 2 here is the Clebbsch of the $U(1) \subset S U(2)$.

* Another way to understand the $\phi<m$ regime - including $\phi \rightarrow 0$ - is via effective SYM theory. Note that for $\phi<m$ the 5D quarks are massive and we may integrate them out. The result is an effective $S U(2)$ SYM with no flavors, $\theta=0$ (cf. eq. (3.30)), and inverse coupling $h^{\text {eff }}=h-m$ (cf. eq. (6.84) for $\phi<m$ ). In terms of the spectral curve integration out works by focusing on $x$ being neither too large nor too small, specifically $e^{-L a M} \ll x \ll e^{+L a M}$; in this regime, the curve (6.60) may be approximated as

$$
y^{2}-y \times\left(x^{2}-2 \cosh (\operatorname{La\varphi }) \times x+1\right)+e^{-L a(H-M)} \times x^{2},
$$

which looks exactly like the curve for $n_{f}^{\text {eff }}=0, \Delta F^{\text {eff }}=2$, and $H^{\text {eff }}=H-M$. And as in $\S 6.2$, this curve yields $T=2 h^{\mathrm{eff}}+8 \phi=2(h-m)+8 \phi$ for $\phi>0$, and for $\phi \rightarrow 0$ and $h^{\text {eff }}>0$ it has two pairs of Seiberg-Witten singularities indicating $S U(2)$ restoration in $5 \mathrm{D}$.

- For $h=m$, the patterns or branching points are similar to the $h>m$ regimes for $\phi>m, \phi=m$, and $0<\phi<m$, but for $\phi \rightarrow 0$ there is a difference: $T \rightarrow 0$ at this point, which indicates a superconformal theory in $5 \mathrm{D}$. The nature of this SCFT is clear from the effective theory - SYM with $\theta=0$ - whose superconformal limit at $h^{\mathrm{eff}}=h-m=0$ and $\phi=0$ is $E_{1}$, exactly as in $\S 6.2$. Moreover, the $E_{1}$ has a Higgs branch growing out of the superconformal point, and the quiver theory does have a 
Higgs branch at precisely this point, namely the exotic baryonic branch with flavors 1 and 3, cf. eq. (6.68).

- For $-m<h<+m$ there are three regimes: for $\phi>m$ the branching points are as in eq. (6.70), for $\frac{m-h}{2}<\phi<m$ they are as in eq. (6.83), and for $0<\phi<\frac{m-h}{2}$ we have a new pattern, namely

$$
x_{1,2}= \pm 2 e^{+L a(M-H) / 2}, \quad x_{3,4}=\mp \frac{1}{2} e^{-L a(M-H) / 2},
$$

with crossratio $\chi=e^{L a(M-H)}$ and hence $T=2(m-h)$, regardless of $\phi$ (as long as $\left.\phi<\frac{m-h}{2}\right)$. Altogether,

$$
\text { for }-m<h<+m, \quad T= \begin{cases}2 h+6 \phi & \text { for } \phi>m, \\ 2 h-2 m+8 \phi & \text { for } \frac{m-h}{2}<\phi<m \\ 2 m-2 h & \text { for } 0<\phi<\frac{m-h}{2}\end{cases}
$$

However, the third regime here is unphysical because the $\mathcal{N}=2$ superpartner $\mathcal{A}$ of the vector field decouples from $\varphi$; indeed, for branching points as in (6.88) we have

$$
\begin{aligned}
\frac{d \mathcal{A}}{d \varphi} & =\frac{2 \sinh (\operatorname{La} \varphi)}{2 \pi i} \times \oint \frac{d x}{\sqrt{\left(x-x_{1}\right)\left(x-x_{2}\right)\left(x-x_{3}\right)\left(x-x_{4}\right)}} \\
& \approx \frac{e^{L a \varphi}}{\sqrt{x_{1} x_{2}}} \\
& \approx \frac{i}{2} e^{L a(2 \varphi+H-M) / 2} \ll 1 .
\end{aligned}
$$

In terms of the effective theory, the decoupling happens for and $2 \phi+h^{\text {eff }}<0$, and it works exactly as in $\S 6.2$. And similar to $\S 6.2$, at the edge of decoupling $\phi=-\frac{1}{2} h^{\text {eff }}=$ $\frac{m-h}{2}$ there is unbroken $S U(2)$ in $5 \mathrm{D}$ - which manifests in $4 \mathrm{D}$ via singularities (6.63) forming two pairs of Seiberg-Witten points,

$$
L a \varphi=L a \frac{M-H}{2}+\log (2)+\{0 \text { or } \pi i\} \pm e^{-L a(M-H) / 2} .
$$

The proper modulus of $S U(2)$ restoration is $\tilde{\phi}=\phi+\frac{h-m}{2}$, or in 5D terms

$$
\cosh (\operatorname{La} \tilde{\varphi})=\cosh (\operatorname{La\varphi }) \times \frac{e^{L a H / 2}}{\sqrt{2 \cosh (\operatorname{LaM})}}
$$


where the hyperbolic cosines indicate that variables $\varphi, M$, and $\tilde{\varphi}$ all live on halfcylinders.

Finally, at $\phi=m$ there is a double flop due to two quarks becoming massless at the same time; the ordinary baryonic branch grows out of this point, $c f$. eq. (6.67).

- For $h=-m$ the intermediate range of $\phi$ disappears and there are only two regimes of the branching points: (6.70) for $\phi>m$ and (6.88) for $\phi<m$, thus

$$
\text { for } h=-m, \quad T= \begin{cases}2 h+6 \phi & \text { for } \phi>m, \\ 4 m & \text { for } 0 \leq \phi<m .\end{cases}
$$

And again, the second regime here is unphysical because $\mathcal{A}$ decouples from $\varphi$. However, the regime boundary at $\phi=m$ is more complicated than for $h>-m$ because now $S U(2)$ restoration happens at the same point where two quarks become massless. In $5 \mathrm{D}$ terms, this corresponds to an effective $D_{2}$ theory with two $m^{\text {eff }}=0$ quarks. In the continuum 5D limit this effective theory should have a global $S O(4)$ symmetry, but the $4 \mathrm{D}$ quiver theory itself is not $S O(4)$ symmetric. Instead, we identify the $h=-m$, $\phi=m$ (i.e., $\tilde{\phi}=0$ ) point as an effective $D_{2}$ origin via singularities of the spectral curve and also via Higgs branches. Indeed, for $H=-M$ the curve (6.60) of our quiver theory has two double $\left(I_{2}\right)$ and two simple $\left(I_{1}\right)$ singularities near $\tilde{\varphi}=0$ or $\frac{\pi i}{L a}$, namely

$$
\begin{aligned}
& \text { first } I_{2} \text { at: } \cosh (\operatorname{La\varphi })=e^{L a M}+\frac{1}{2} e^{-L a M} \Longrightarrow(\operatorname{La\tilde {\varphi }})^{2} \approx \frac{1}{4} e^{-4 L a M}, \\
& \text { second } I_{2} \text { at: } \cosh (\operatorname{La\varphi })=e^{L a M} \quad \Longrightarrow(\operatorname{La\tilde {\varphi }})^{2} \quad \approx-e^{-2 L a M} \text {, }
\end{aligned}
$$

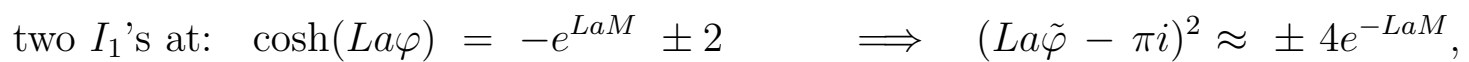

and this is precisely the singularity structure of $D_{2}, c f$. eqs. (6.72-73). Moreover, there are two distinct Higgs branches rooted at the double singularities near $\tilde{\varphi}=0$, namely the ordinary baryonic branch $B_{12}$ rooted at the first $I_{2}$ (cf. eq. (6.65) for $\left.e^{-L a H}=e^{+L a M}\right)$, and the exotic baryonic branch $B_{23}$ rooted at the second $I_{2}(c f$. eq. (6.64)). In terms of the effective $D_{2}$ theory, one of these Higgs branches corresponds to the mesonic branch and the other to the baryonic branch. Altogether, the 4D singularities and the Higgs branches confirm our identification of the $\phi=-h=m$ point as the $D_{2}$ theory in $5 \mathrm{D}$. 
- Finally, for $h<-m$ we have three regimes: for $\phi>-h$, the branching points of the spectral curve as in eq. (6.70); for $\frac{m-h}{2}<\phi<-h$ we have a new pattern

$$
x_{1} \approx-4 e^{-L a H}, \quad x_{2} \approx-\frac{1}{4} e^{L a(H+2 \varphi)}, \quad x_{3} \approx-4 e^{-L a(H+2 \varphi)}, \quad x_{4} \approx-\frac{1}{4} e^{+L a H}
$$

with crossratio $\chi=\frac{1}{16} e^{L a(2 H+4 \varphi)}$; and for $0 \leq \phi<\frac{m-h}{2}$ the branching points are

$$
x_{1} \approx-4 e^{-L a H}, \quad x_{2} \approx-e^{L a M}, \quad x_{3} \approx-e^{-L a M}, \quad x_{4} \approx-\frac{1}{4} e^{+L a H}
$$

with crossratio $\chi=e^{2 L a M}$, regardless of $\varphi$. Altogether, this gives us

$$
\text { for } h<-m, \quad T= \begin{cases}2 h+6 \phi & \text { for } \phi>-h \\ 4 h+8 \phi & \text { for } \frac{m-h}{2}<\phi<-h \\ 4 m & \text { for } 0 \leq \phi<\frac{m-h}{2}\end{cases}
$$

Note that the third regime here is unphysical because for branching points (6.97)

$$
\begin{aligned}
\frac{d \mathcal{A}}{d \varphi} & \approx \frac{e^{L a \varphi}}{\sqrt{x_{1} x_{2}}} \\
& \approx \frac{1}{2} e^{L a(H-M+2 \varphi) / 2} \ll 1 .
\end{aligned}
$$

In other words, the proper 5D modulus is not $\phi \geq 0$ but $\tilde{\phi}=\phi-\frac{m-h}{2} \geq 0$. Moreover, at the endpoint $\tilde{\phi}=0$ there is unbroken $S U(2)$ in $5 \mathrm{D}$ as evidenced by singularities (6.63) of the spectral curve forming two Seiberg-Witten pairs

$$
\begin{gathered}
L a \varphi=L a \frac{M-H}{2}+\log (2)+\{0 \text { or } \pi i\} \pm e^{-L a M}, \\
(L a \tilde{\varphi})^{2} \quad \text { or } \quad(L a \tilde{\varphi}-\pi i)^{2}= \pm 2 e^{-2 L a M} .
\end{gathered}
$$

Finally, at $\phi=-h$ - which corresponds to $\tilde{\phi}=\frac{-h-m}{2}>0$ - there is a double flop transition due to two quark flavors becoming massless. As usual, such double flop is root of the ordinary baryonic branch $B_{12}$, cf. eq. (6.67) for $h<-m$. 
Altogether, various regimes of our model are summarized on the following diagram:

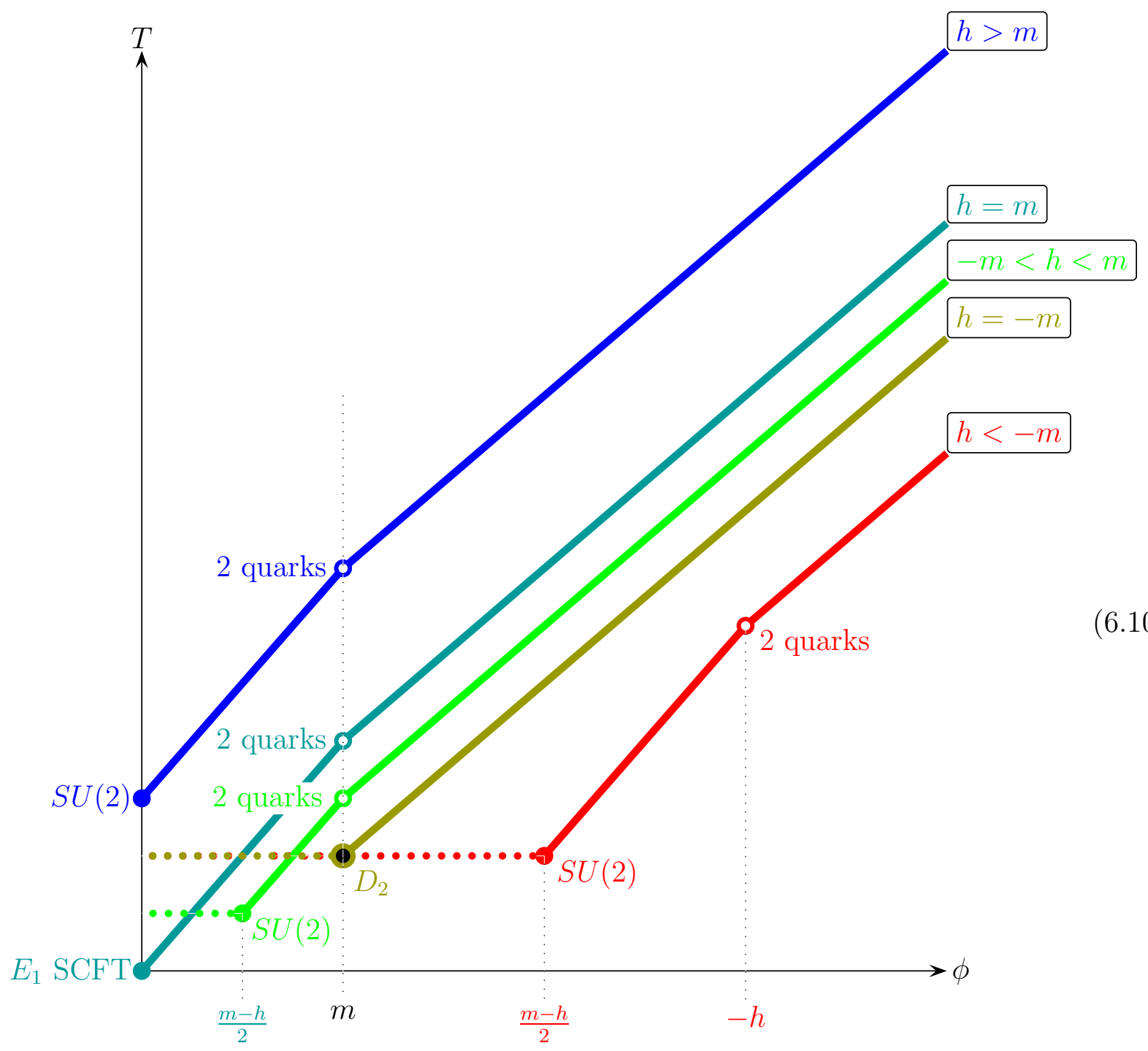

The colored lines here plot $T(\phi)$ for several fixed values of $h$ (different colors for different $h)$; the solid lines correspond to physical 5D regimes, and the dotted lines to unphysical regimes for $\phi<\frac{m-h}{2}$ where $\varphi$ decouples from the low-energy degrees of freedom. Note the blue, cyan, and green lines are bent at $\phi=m$ : the slope $d T / d \phi$ is 6 for $\phi>m$ and 8 for $\phi<m$. Likewise, the red line is bend at $\phi=-h$; the slope is 6 for $\phi>-h$ and 8 for $\phi<-h$. In terms of the Seiberg formula (6.7), slope $=6$ corresponds to Coulomb branch of the $D_{2}$ theory, while slope $=8$ corresponds to Coulomb branch of an effective SYM theory. Thus, we deconstruct the following Coulomb phase diagram of the $S U(2)$ theory with $n_{f}=2$, 
$\Delta F=1$, and fixed $m_{2}=-m_{1}=m>0$ :

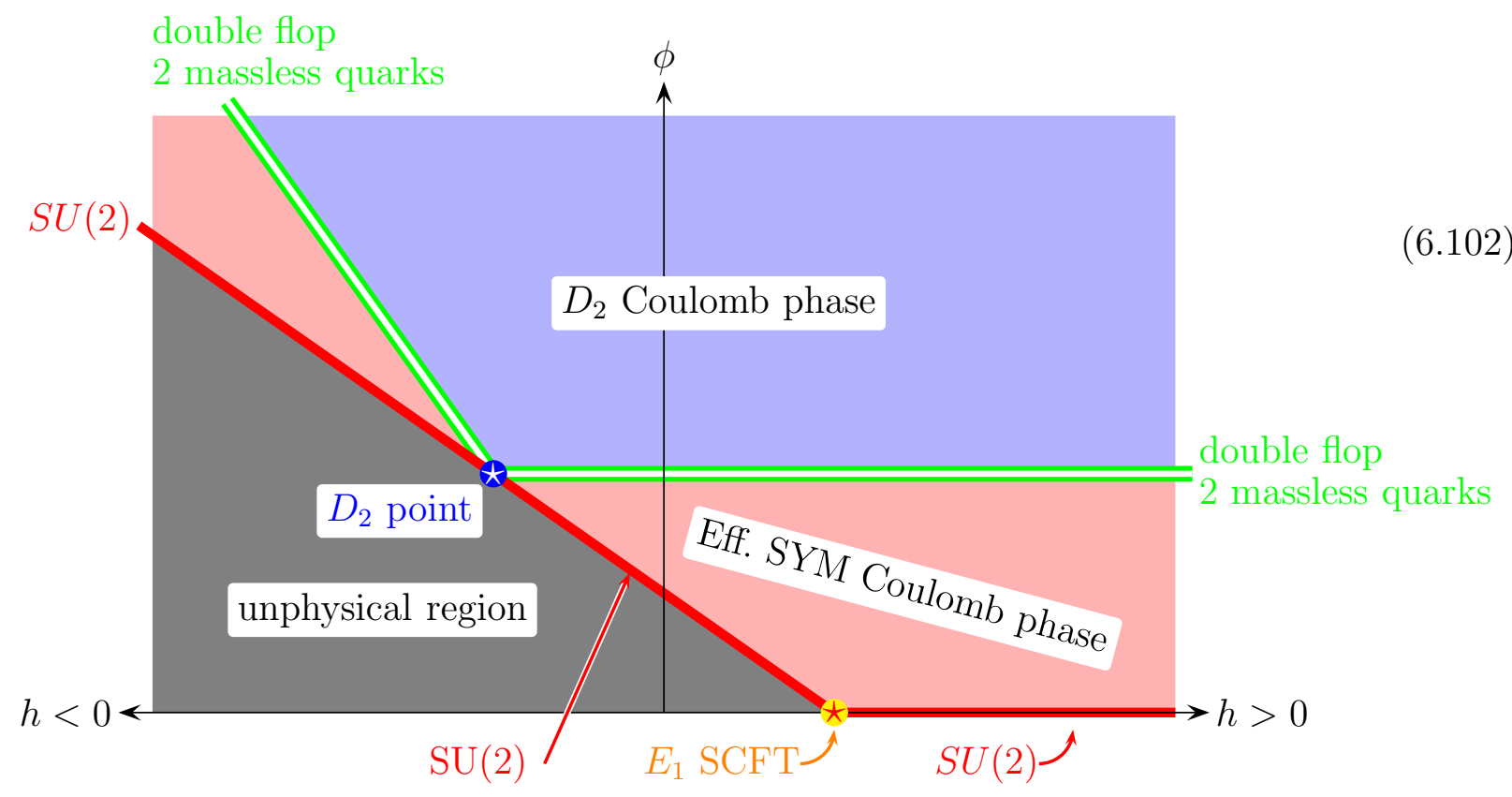

The effective SYM for $h>-m$ and $\phi<m$ is $D_{0}$ : it has $\theta=0$ in $5 \mathrm{D}$, and in $4 \mathrm{D}$ the effective curve (6.87) has $\Delta F^{\mathrm{eff}}=0$. The other effective SYM for $h<-m$ and $\phi<-h$ is also $D_{0}$, and in fact at the spectral curve level, there is a symmetry between the two $D_{0}$ phases. To make this symmetry manifest, we change the $y$ variable in eq. (6.60) to $\bar{y}=-y /\left(x-e^{-\operatorname{LaM}}\right)$ and then rewrite the spectral curve as

$\bar{y}^{2} \times\left(x-e^{-L a M}\right)+\bar{y} \times\left(x^{2}-2 \cosh (\operatorname{La\varphi }) \times x+1\right)-e^{-L a H} \times x \times\left(x-e^{+L a M}\right)=0$,

or equivalently

$x \bar{y}(x+\bar{y})-\left(e^{-L a H} \times x^{2}+e^{-L a M} \times \bar{y}^{2}+2 \cosh (L a \varphi) \times x \bar{y}\right)+\left(e^{L a(M-H)} \times x+y\right)=0$.

The resulting equation is invariant under

$$
\begin{aligned}
& x \rightarrow \bar{y} \times e^{L a(M-H) / 2}, \quad \bar{y} \rightarrow x \times e^{L a(M-H) / 2}, \\
& H \rightarrow \frac{3 M-H}{2}, \quad M \rightarrow \frac{M+H}{2}, \quad \cosh (\operatorname{La\varphi }) \rightarrow \cosh (\operatorname{La\varphi }) \times e^{L a(H-M) / 2},
\end{aligned}
$$

and this symmetry indeed interchanges the two effective $D_{0}$ phases on the Coulomb phase 
diagram (6.102). Note however that this is not a symmetry of the quiver theory itself but only of its spectral curve.

As to the Higgs branches of the deconstructed $S U(2)$ theory with $n_{f}=2, \Delta F=1$, and $m_{2}=-m_{1}=m>0$, there is the ordinary baryonic branch $B_{12}$ rooted along the double-flop line $\phi=\max (m,-h)$, and two exotic baryonic branches: $B_{13}$ rooted at the $E_{1}$ SCFT point $h=+m, \phi=0$ and $B_{23}$ rooted at the $D_{2}$ point $\phi=-h=m$. There are no mesonic or mixed Higgs branches for $m \neq 0$.

And now we move on to another example of deconstructed $\mathrm{SQCD}_{5}$ with $n_{c}=n_{f}=2$. This time, we take $\Delta F=0$ rather than 1 , and impose a different constraint on the quark masses, namely $m_{1}=m_{2}=\tilde{m}$, or in $4 \mathrm{D}$ terms $\mu_{1}=\mu_{2}=V e^{a \tilde{M}} \cdot{ }^{\langle 14\rangle}$ This choice gives us a quiver with manifest $U(2)$ flavor symmetry. On the other hand, flavor symmetry enhancement $U(2) \rightarrow S O(4)$ in $5 \mathrm{D}$ for $M=0$ is not protected by the discrete custodial symmetry $\mathbf{C}$ (4.25-29) because $\Delta F \neq 1$ breaks $\mathbf{C}$.

The spectral curve of the $\Delta F=0$ quiver is

$$
\tilde{y}^{2}-\tilde{y} \times\left(\tilde{x}^{2}-2 \cosh (\operatorname{La} \tilde{\varphi}) \times \tilde{x}+1\right)+e^{-L a \tilde{S}} \times\left(\tilde{x}-e^{L a \tilde{M}}\right)^{2}=0
$$

where $\tilde{S}=\tilde{H}+\tilde{M}$ (cf. eq. (4.28)) and $\operatorname{Re} \tilde{H} \geq \operatorname{Re} \tilde{M}$ (cf. eq. (4.53). Consequently, for $\tilde{m} \equiv \operatorname{Re} \tilde{M}>0$ we expect no phase transitions (except for a double flop at $\tilde{\phi}=\tilde{m}$ ), but for $\tilde{m}<0$ there should be distinct phases for $\tilde{h} \equiv \operatorname{Re} \tilde{H}>-\tilde{m}$ and for $\tilde{m}<\tilde{h}<-\tilde{m}$.

The curve (6.106) factorizes for $\tilde{\varphi}= \pm \tilde{M}$ where the quiver has a mesonic Higgs branch, and also for

$$
\cosh (\operatorname{La\tilde {\varphi }})=e^{L a \tilde{M}}, \quad e^{2 L a \tilde{M}}+e^{-L a(\tilde{H}+\tilde{M})}=1
$$

where the quiver has a baryonic Higgs branch $B_{12}$. This is the only baryonic branch for this quiver: because of $\Delta F=0$ there are no exotic branches. In fact, there are no Higgs branches

$\langle 14\rangle$ For this model, we put tildes on all the variables: $\tilde{M}, \tilde{H}, \tilde{\varphi}$, etc., etc. Such notations help discuss duality (6.109) betwen spectral curves of this model and the previous model with $\Delta F=1$. The tildes make clear which variable belongs to which model. 
other than the mesonic and the (ordinary) baryonic branches; in the 5D limit La $\rightarrow \infty$ they are located at:

$$
\begin{array}{ll}
\text { mesonic: } & \tilde{\phi}=|\tilde{m}|, \\
\text { baryonic: } & \tilde{\phi}=0, \quad \tilde{h} \geq 0, \quad \tilde{m}=0 \text { or } \tilde{m}=-\tilde{h} .
\end{array}
$$

We may analyze the Coulomb branch of the present quiver in the usual way, by studying the branching points of the curve (6.106), but there is an easier way. The curve (6.106) happens to be dual to the curve (6.60) of the previous model:

$$
\begin{aligned}
& y^{2}-y \times\left(x^{2}-2 \cosh (\operatorname{La\varphi }) \times x+1\right)-e^{-L a H} \times x \times\left(x^{2}-2 \cosh (\operatorname{LaM}) \times x+1\right)=0 \\
& \tilde{y}^{2}-\tilde{y} \times\left(\tilde{x}^{2}-2 \cosh (\operatorname{La} \tilde{\varphi}) \times \tilde{x}+1\right) \stackrel{\Uparrow}{\Downarrow}+e^{-L a \tilde{S}} \times\left(\tilde{x}-e^{L a M}\right)^{2}=0 \\
& \text { for } \quad \tilde{y}=\frac{y+e^{-L a H} x}{2 \cosh (\operatorname{LaM})}, \quad \tilde{x}=-\frac{y}{x} \times \frac{e^{+L a H / 2}}{\sqrt{2 \cosh (\text { LaM })}}, \\
& e^{-L a \tilde{S}}=\frac{e^{+L a H}}{2 \cosh (\operatorname{LaM})}, \quad e^{L a \tilde{M}}=\frac{e^{-L a H / 2}}{\sqrt{2 \cosh (\text { LaM })}}, \\
& \text { and } \cosh (\operatorname{La\tilde {\varphi }})=\cosh (\operatorname{La\varphi }) \times \frac{e^{L a H / 2}}{\sqrt{2 \cosh (L a M)}}, \quad c f . \text { eq. (6.93). }
\end{aligned}
$$

Physically, the two $n_{c}=n_{f}=2$ quiver theories are not dual to each other; they are not even in the same universality class. Indeed, the Higgs branches of the two theories are not quite dual to each other:

$$
\begin{aligned}
n_{c}=n_{f}=2, \Delta F=1 \text { quiver } & \longleftrightarrow n_{c}=n_{f}=2, \Delta F=0 \text { quiver } \\
B_{12} \text { baryonic branch } & \longleftrightarrow \text { mesonic branch, } \\
B_{13} \text { and } B_{23} \text { baryonic branches } & \longleftrightarrow \text { baryonic branch(es), } \\
\text { mesonic branch } & \longleftrightarrow \text { nothing. }{ }^{15\rangle}
\end{aligned}
$$

However, their spectral curves are dual, and we may use use this duality to obtain the

$\langle 15\rangle$ The spectral curve (6.60) of the $\Delta F=1$ quiver has an $I_{2}$ singularity at the mesonic root $\varphi=M=0$, and the duality maps it onto a similar $I_{2}$ singularity of the $\Delta F=0$ quiver's spectral curve at $\cosh (\operatorname{La} \tilde{\varphi})=$ $\frac{1}{2} e^{-L a \tilde{M}}=2 e^{-L a \tilde{H}}$. However, despite this singularity, the curve (6.106) does not factorize. Also, at this point, the link resolvent $T(\tilde{X})$ of the $\Delta F=0$ quiver has no poles at the physical sheet; instead, there is a pole on the unphysical sheet with residue $=2$. Anyhow, there is no Higgs branch at this point. 
Coulomb phase diagram of our second $n_{c}=n_{f}=2$ example without too much work.

In the $5 \mathrm{D}$ limit $L a \rightarrow \infty$, the duality map (6.109) becomes

$$
\tilde{h}=\frac{3|m|-h}{2}, \quad \tilde{m}=\frac{-|m|-h}{2}, \quad|\tilde{\phi}|=|\phi|+\frac{h-|m|}{2} .
$$

Note that according to this map, there is a lower limit $\tilde{h} \geq \tilde{m}$; remarkably, this limit agrees with eq. (4.53) which follows from very different physics, namely quantum corrections $V=v+\cdots$ in a quiver without $\mu=0$ quarks. This agreement indicates that the map (6.111) covers all physical Coulomb phases of the two theories (even though it misses some of the Higgs phases). Consequently, applying this map to diagrams (6.80) and (6.102) of the $\Delta F=1$ model, we arrive at the following Coulomb phase diagram of deconstructed $\mathrm{SQCD}_{5}$ with $n_{c}=n_{f}=2, \Delta F=0$, and $m_{1}=m_{2}=\tilde{m}$ :

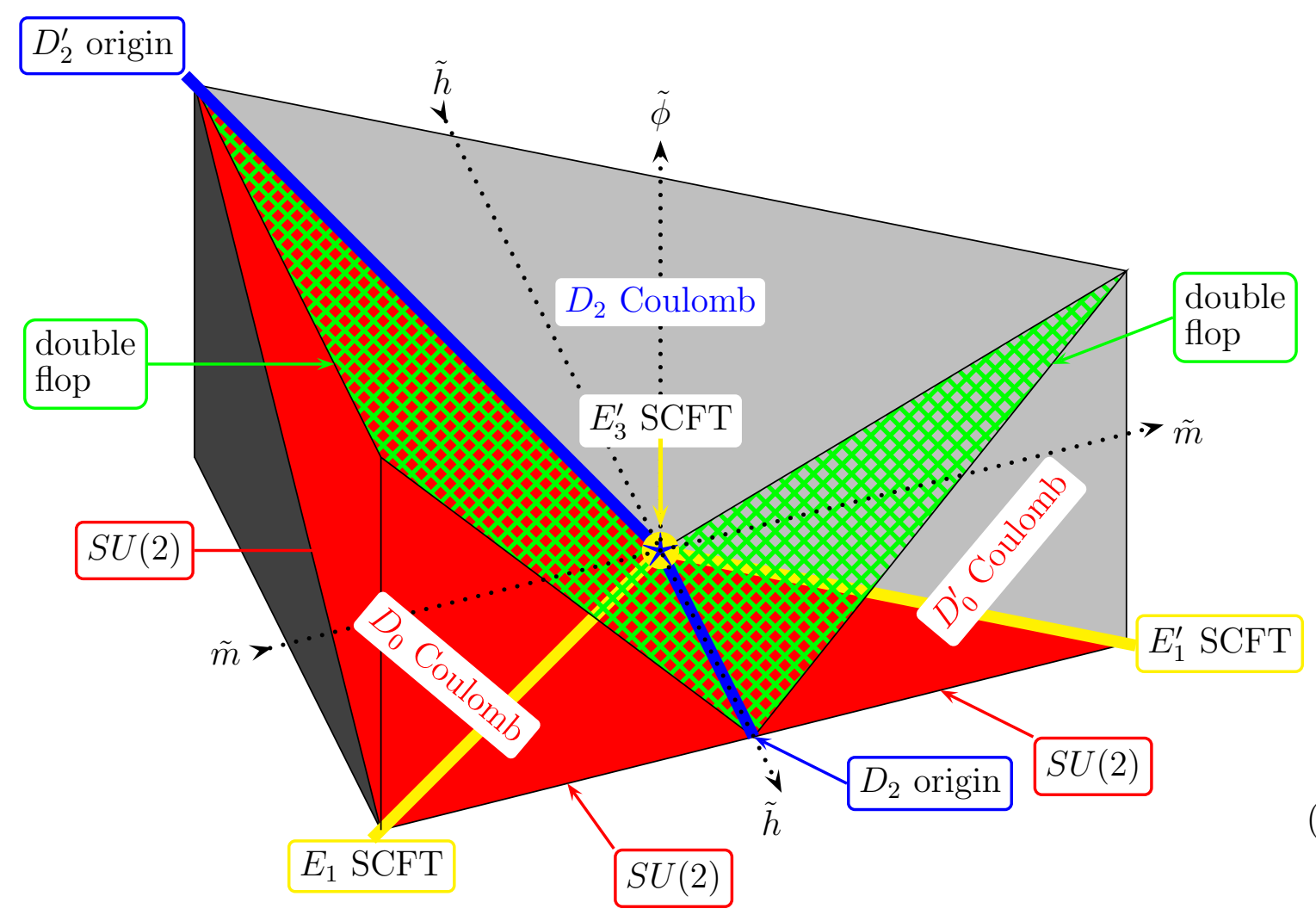

This diagram shows 3D parameter/moduli space spanned by $\tilde{m}, \tilde{h}$, and $\tilde{\phi}$. There are two unphysical regions $-\tilde{h}<\tilde{m}$ and $2 \tilde{\phi}+\tilde{h}+\tilde{m}<0-$ and three distinct physical regions 
separated by double flop transitions at $\tilde{\phi}=|\tilde{m}|$ :

(1) The $D_{2}$ Coulomb phase for $\tilde{\phi}>|\tilde{m}|$ where $T=2 \tilde{h}+6 \tilde{\phi}$.

(2) The effective $D_{0}(\mathrm{SYM})$ Coulomb phase for $\tilde{m}<0$ and $\tilde{\phi}<|\tilde{m}|$ where $T=2 \tilde{h}^{\text {eff }}+8 \tilde{\phi}$. In quiver terms, in this phase the quark bare mass $\tilde{\mu}=V e^{a \tilde{m}}$ is effectively $\tilde{\mu} \approx 0$, hence $n_{f}^{\text {eff }}=0$ but $\Delta F^{\text {eff }}=2$, and everything works as in $\S 6.2$. In particular, there is $S U(2)$ restoration for $\tilde{\phi}=0$ and $\tilde{h}^{\text {eff }}=\frac{\tilde{h}+\tilde{m}}{2}>0$, and also for $\tilde{h}^{\text {eff }}<0$ and $\tilde{\phi}=-\tilde{h}^{\text {eff }} / 2$ (this is dual to $\phi=0)$. And for $\tilde{h}^{\text {eff }}=0$ and $\tilde{\phi}=0$ we have $E_{1}$ SCFT.

(3) The $D_{0}^{\prime}$ Coulomb phase for $\tilde{m}>0$ and $\tilde{\phi}<\tilde{m}$. This is another effective SYM Coulomb phase with $T=2 \tilde{h}^{\text {eff }}+8 \tilde{\phi}$ and $S U(2)$ restoration for $\tilde{\phi}=0$. However, this time $\tilde{h}^{\text {eff }}=\frac{\tilde{h}-\tilde{m}}{2}$ is always nonnegative. In quiver terms, in this phase $\tilde{\mu}=V e^{a \tilde{m}}$ is so large the quarks effectively decouple, hence $n_{f}^{\text {eff }}=\Delta F^{\text {eff }}=0$; this effective theory works as in [20]. In particular, for $\tilde{h}^{\text {eff }}=0$ and $\tilde{\phi}=0$ there is a 5D SCFT.

We call this superconformal theory $E_{1}^{\prime}$ because its spectral curve is dual to the curve of the $E_{1}$; in particular, there are two $I_{1}$ singularities and one $I_{2}$. However, there are major differences between the two SCFTs: the $E_{1}^{\prime}$ does not have a Higgs branch, and its Coulomb branch is limited to $\tilde{h}^{\text {eff }} \geq 0$. In $\mathrm{M}$ theory, the $E_{1}^{\prime}$ SCFT arises from a Calabi-Yau with a $\mathbb{C}^{3} / \mathbb{Z}_{4}$ orbifold singularity. Note that such singular points are not isolated but lie on lines of milder $A_{1}$ singularity $\left(\mathbb{C}^{2} / \mathbb{Z}_{2}\right)$, and the line cannot be blown up without also blowing up the point. This is unlike $E_{1}$ which arises from an isolated singular point, namely conifold $/ \mathbb{Z}_{2}$.

The three Coulomb phases come together at $\tilde{\phi}=\tilde{m}=0, \tilde{h}>0$, where we have a $D_{2}$ theory at its origin - unbroken $S U(2)$ and two massless quarks at the same time. Likewise, there is unbroken $S U(2)$ and two massless quarks along the $\tilde{\phi}=-\tilde{h}=-\tilde{m}>0$ line, which we call the ' $D_{2}^{\prime}$ origin'. From the spectral curve's point of view, the $D_{2}$ and the $D_{2}^{\prime}$ origins have similar singularity structures $2 I_{2}+2 I_{1}$. But beyond the spectral curve level, the two origins origin are different. In particular, there are three Coulomb phases near the $D_{2}$ origin but only two Coulomb phases - the $D_{2}$ and the $D_{0}$ - near the $D_{2}^{\prime}$ origin; the $D_{0}^{\prime}$ Coulomb phase is cut off by the deconstruction limit $\tilde{h} \geq \tilde{m}$. Likewise, two Higgs branches - one 
mesonic and one baryonic - have roots at the $D_{2}$ origin, but the $D_{2}^{\prime}$ origin has only the mesonic Higgs branch.

Indeed, here is the diagram of the Higgs phases (6.108) of the $\Delta F=0$ theory:

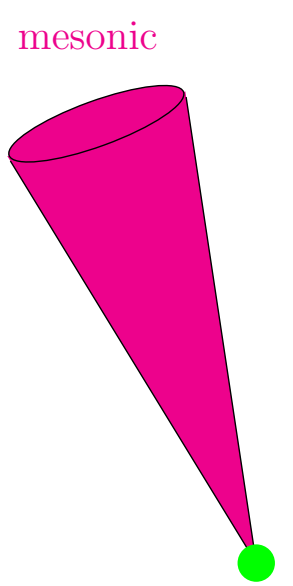

double flop

$\tilde{\phi}= \pm \tilde{m}$

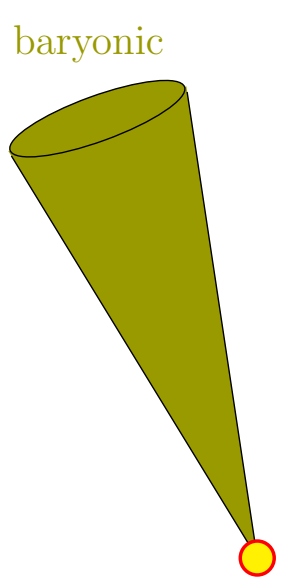

mesonic

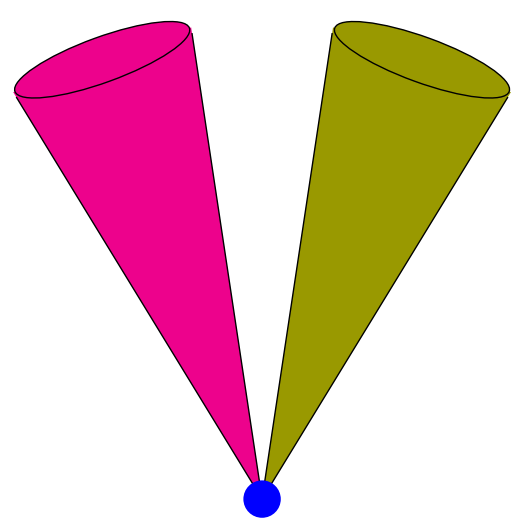

$D_{2}$ origin

$\tilde{\phi}=\tilde{m}=0, \tilde{h}>0$ mesonic missing

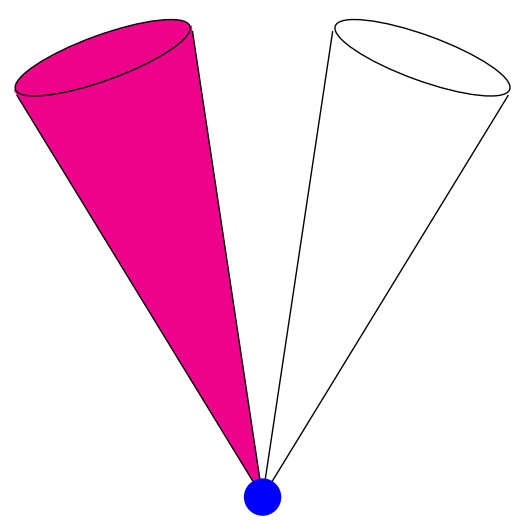

$D_{2}^{\prime}$ origin

$\tilde{\phi}=-\tilde{m}=-\tilde{h}>0$
$E_{1} \mathrm{SCFT}$

baryonic

$\tilde{\phi}=0, \tilde{h}=-\tilde{m}>0$ missing

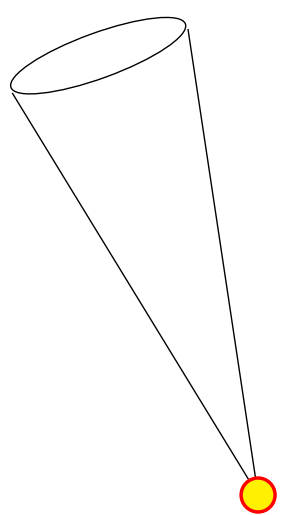

$E_{1}^{\prime} \mathrm{SCFT}$

mesonic

baryonic, unmixed
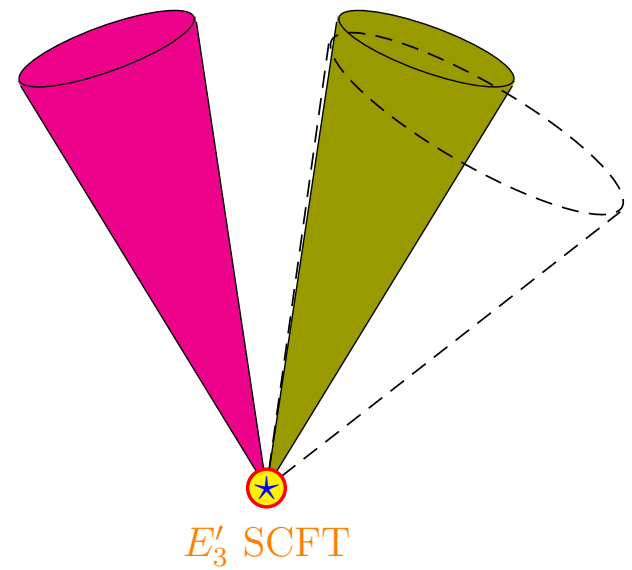

$\tilde{\phi}=\tilde{m}=\tilde{h}=0$

where "missing" branches do not exist for $\Delta F=0$ but their roots are dual to roots of mesonic branches of the $\Delta F=1$ theory. And the dashed lines making an empty wide cone around the last Higgs branch here indicate that the branch has hypermultiplet dimension $=1$ - like all the other Higgs branches of the $\Delta F=0$ theory - but in the dual $\Delta F=1$ theory there is a Higgs branch of dimension $=2$, cf. diagram (6.82). 
The central point $\tilde{\phi}=\tilde{m}=\tilde{h}=0$ of the Coulomb phase diagram $(6.112)$ - where the last pair of Higgs branches (6.113) are rooted - is dual to the $E_{3}$ SCFT point of the $\Delta F=1$ theory. For $\Delta F=0$ this point is also superconformal; we call this SCFT $E_{3}^{\prime}$ because of the duality, and also because it is similar to $E_{3}$ SCFT in many ways: (1) the $E_{3}^{\prime}$ obtains in the $\tilde{h} \rightarrow 0$ limit of $D_{2} ;(2)$ its spectral curve in $4 \mathrm{D}$ has $I_{3}+I_{2}+I_{1}$ singularities; (3) it has two distinct Higgs branches. However, the dimensions of the Higgs branches are different: $1+1$ (in hypermultiplet units) for the $E_{3}^{\prime}$ versus $1+2$ for the $E_{3}$. In $\mathrm{M}$ theory, the $E_{3}$ and the $E_{3}^{\prime}$ SCFTs are realized on Calabi-Yaus with different singularity types. There respective toric diagrams are:
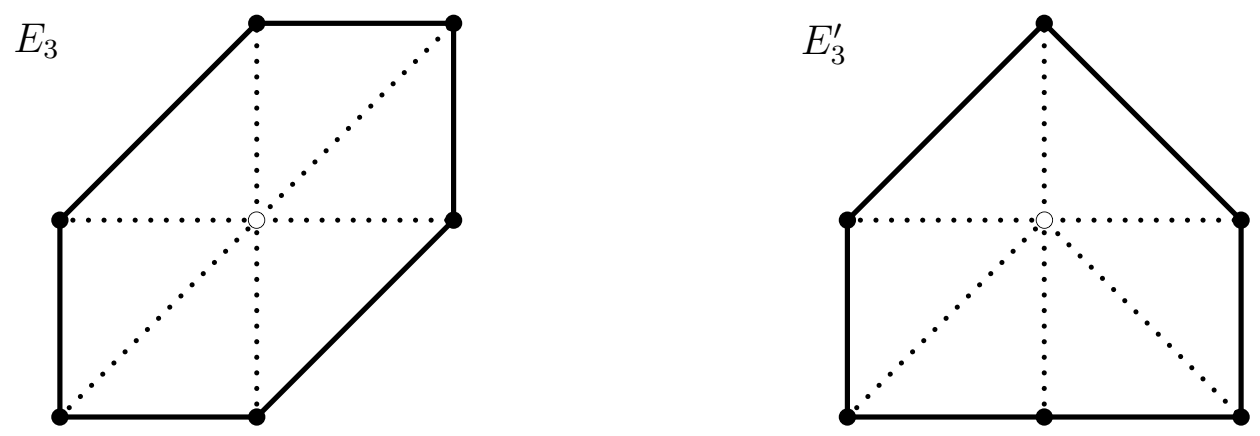

Note the lower edge of the $E_{3}^{\prime}$ diagram has a middle point: this indicates that the singularity is not an isolated point but a more-singular point on a less-singular line, and the line cannot be blown up without blowing up the point at the same time.

In type IIB string theory, the brane webs for the $E_{3}$ and the $E_{3}^{\prime}$ are as follows: for the SCFT points themselves
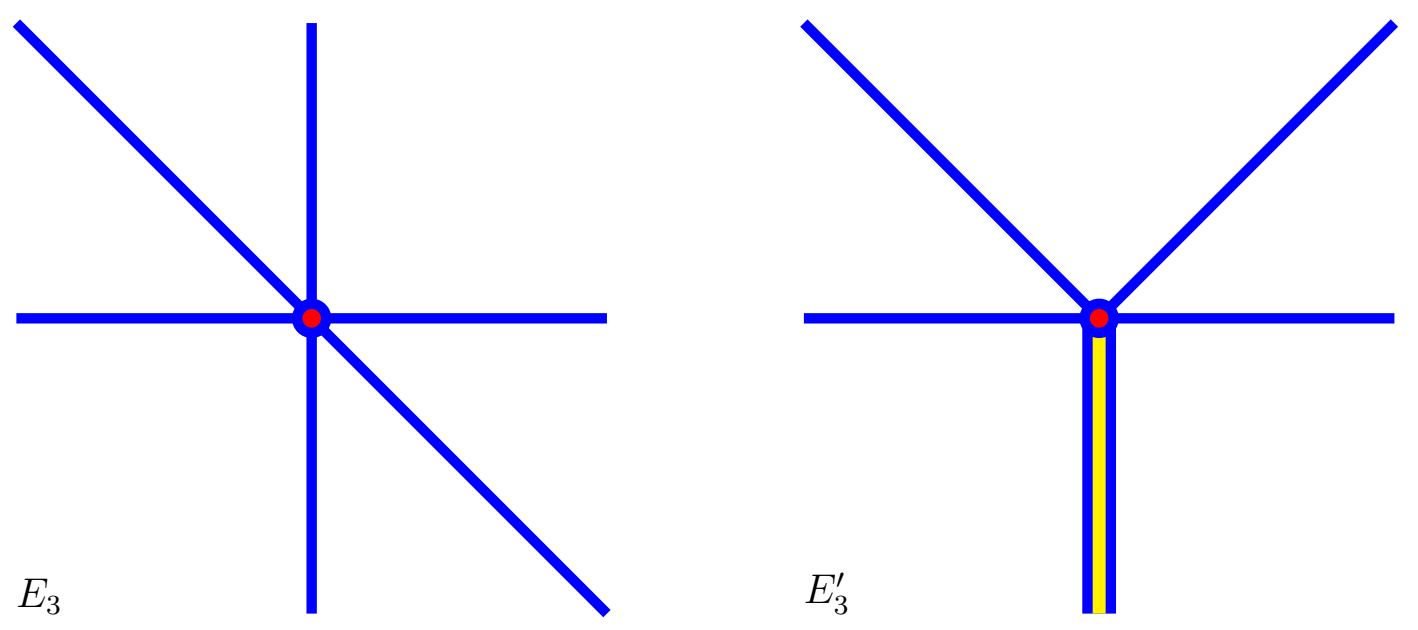
for the respective Coulomb branches $(m=h=0$ but $\phi>0$ or $\tilde{m}=\tilde{h}=0$ but $\tilde{\phi}>0)$
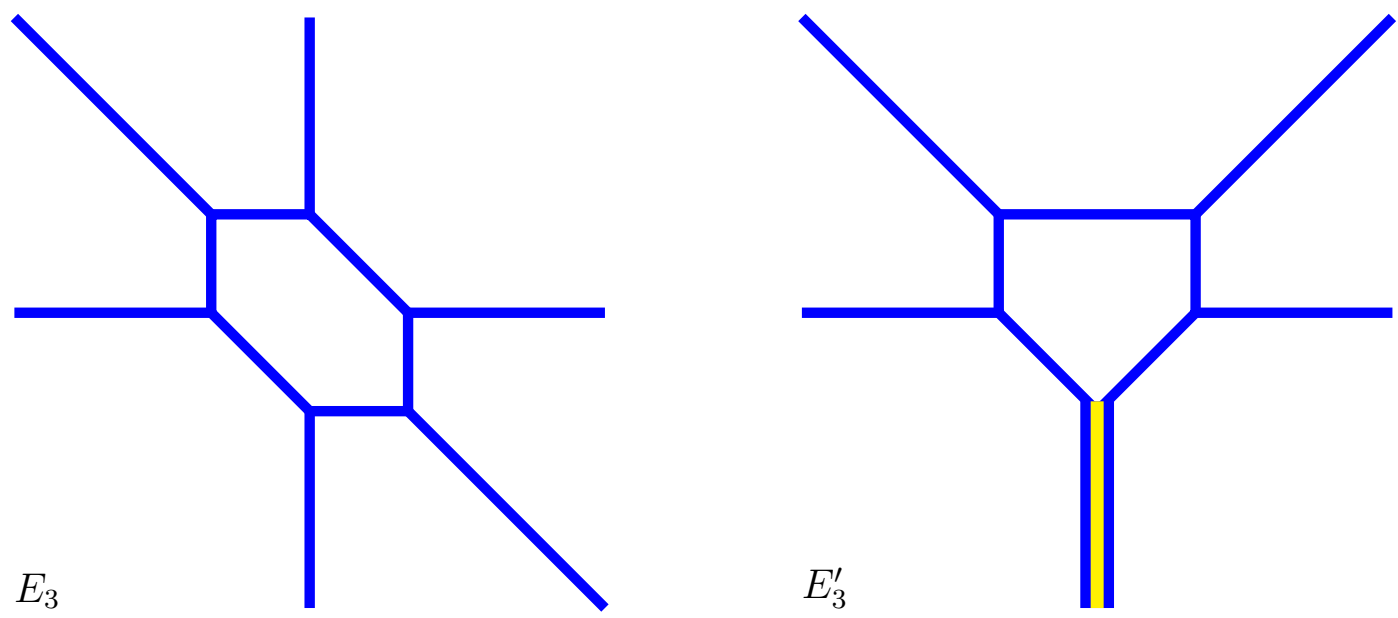

for the baryonic Higgs branches
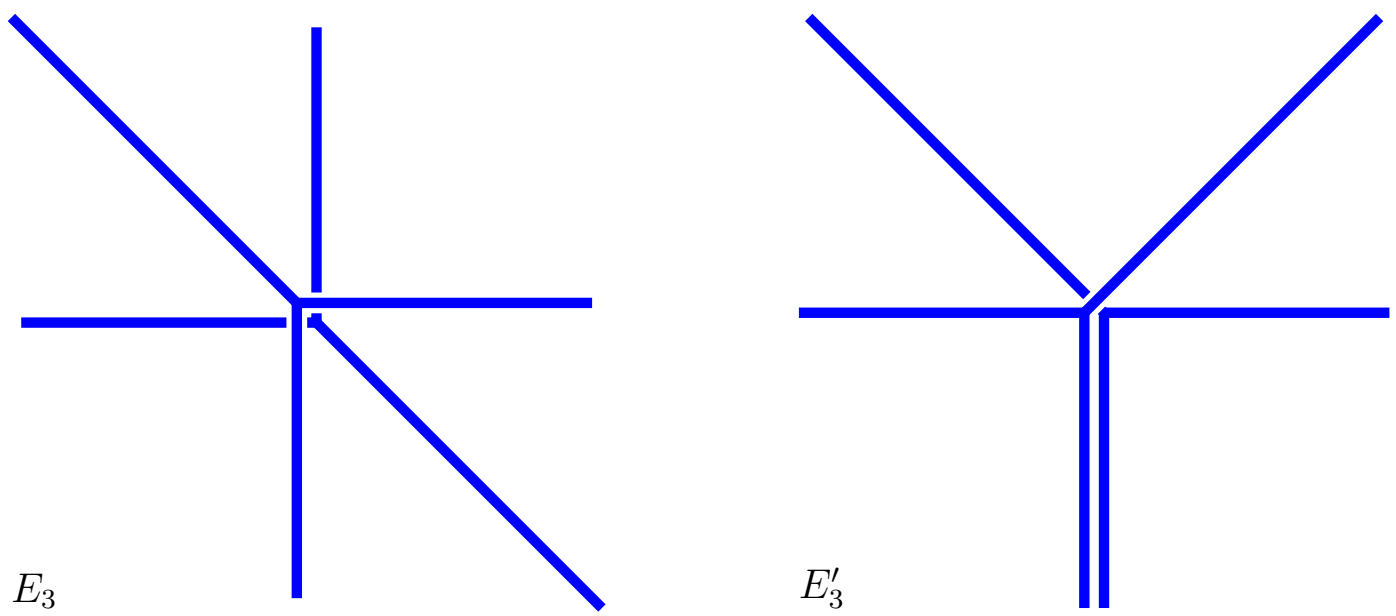

for the mixed / mesonic Higgs branches
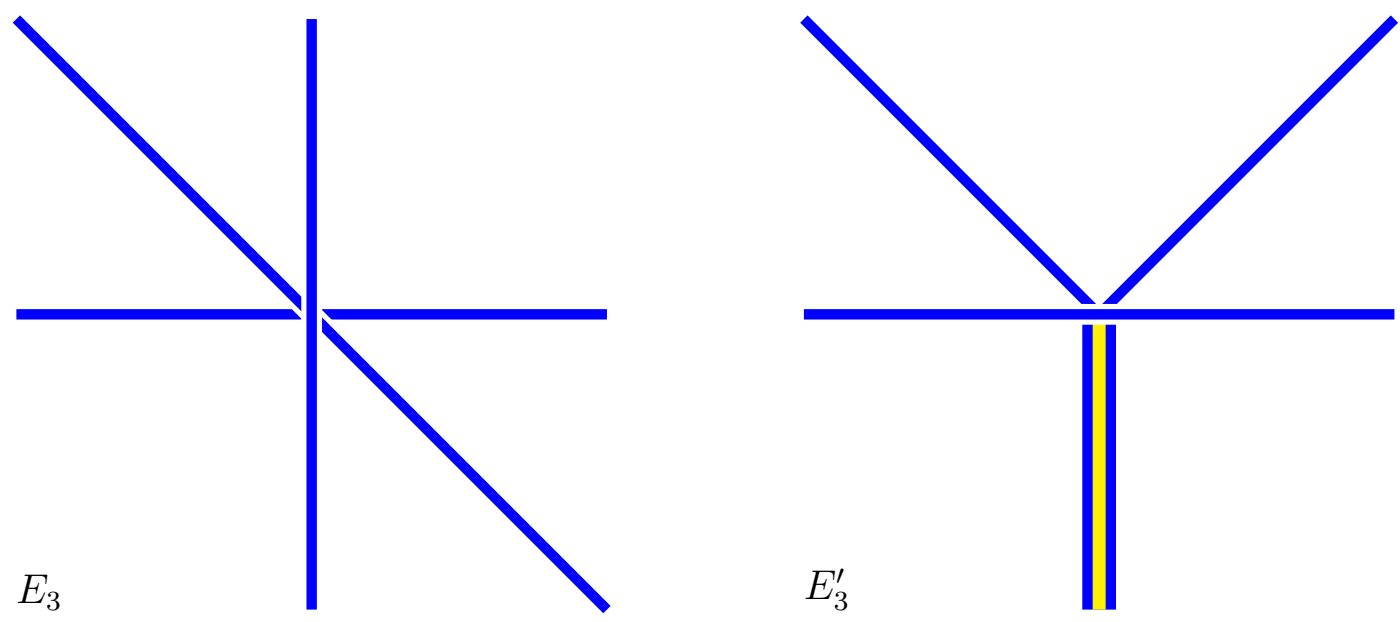
Note the web for the mixed branch of $E_{3}$ has three disconnected lines: this corresponds to hypermultiplet dimension $=2$. In comparison, the webs for baryonic branches of both SCFTs and also for the mesonic branch of $E_{3}^{\prime}$ have only two disconnected pieces each: this corresponds to dimension $=1$.

And of course there are many more webs for non-conformal values of the Coulomb parameters $m \neq 0$ and/or $h \neq 0$ (or $\tilde{m} \neq 0$ and/or $\tilde{h} \neq 0$ ). In fact, there too many webs, so we don't diagram them here. Instead, let us simply state the main result: The brane webs for the $E_{3}$ and its resolutions and deformations have precisely the same physical phases both Coulomb and Higgs - as the deconstructed $\mathrm{SQCD}_{5}$ with $n_{c}=n_{f}=2$ and $\Delta F=1$. Likewise, the webs for the $E_{3}^{\prime}$ and its resolutions and deformations have precisely the same physical phases as the deconstructed $\mathrm{SQCD}_{5}$ with $n_{c}=n_{f}=2$ and $\Delta F=0$. And in both cases, the webs corresponding to the unphysical phases cannot be built. In other words, in both cases, the brane webs in string theory are in perfect agreement with the dimensional deconstruction. 


\section{Deconstruction / String Universality}

In the last section we saw four examples of non-perturbative phase diagrams, and in all four cases dimensional deconstruction and type IIB brane web implementation of the same 5D theory yielded identical diagrams (except for the non-geometric phases of unphysical regions of the moduli/parameter space). In this section, we shall see that such deconstruction / string universality is general and holds for any $n_{c}, n_{f}$, and $k_{\mathrm{cs}}$. Specifically, deconstructive and brane-web completions of the same $\mathrm{SQCD}_{5}$ are in the same universality class and have similar moduli/parameter spaces and similar prepotentials $\mathcal{F}\left(\phi_{1}, \ldots, \phi_{n_{c}} ; h ; m_{1}, \ldots, m_{n_{f}}\right)$. However, the two completions are not dual to each other and become dissimilar outside the zero-energy limit. This is similar to the universality between the 4D SQCD and the MQCD $[26,27,28]$ : they are not dual to each other but are in the same universality class and have similar holomorphic properties.

In fact, the 5D universality between deconstruction and brane webs is based on the 4D universality between SQCD and MQCD, or rather its generalization to more complicated $4 \mathrm{D}$ theories. Specifically, we start a deconstructed $\mathrm{SQCD}_{5}$, treat it as a $4 \mathrm{D}\left[S U\left(n_{c}\right)\right]^{L}$ quiver theory, and take its M-theory counterpart: an M5 brane spanning the 4D Minkowski space and the quiver's spectral curve (4.21). We are going to take the large $L$ limit of this correspondence, so instead of identifying the $x$ and $y$ coordinates of the spectral curve with some of the 7 extra dimensions of the $\mathrm{M}$ theory, we embed them in a non-linear manner based on eqs. (4.30), namely

$$
\begin{array}{ll}
x=\exp (\operatorname{La} \times \xi), & \xi=\frac{X^{5}+i X^{9}}{C}, \\
y=\exp (L a \times \eta), & \eta=\frac{X^{6}+i X^{10}}{C}
\end{array}
$$

where $C$ is a constant parameter, to be determined later in this section. Consequently, the induced metric on the M5 brane itself is

$$
d s^{2}=d X_{0123}^{2}+C^{2}(d \bar{\xi} d \xi+d \bar{\eta} d \eta)=d X_{0123}^{2}+\frac{C^{2}}{(L a)^{2}}\left(\frac{d \bar{x} d x}{|x|^{2}}+\frac{d \bar{y} d y}{|y|^{2}}\right)
$$

where $x$ and $y$ are related according to eq. (4.21). 
We claim that the $L a \rightarrow \infty$ limit of this M5 brane is dual to a 5D brane web, and moreover this web implements the very $\mathrm{SQCD}_{5}$ we have started from. Combining this duality with the generalized SQCD/MQCD duality in 4D, we arrive at the following diagram:

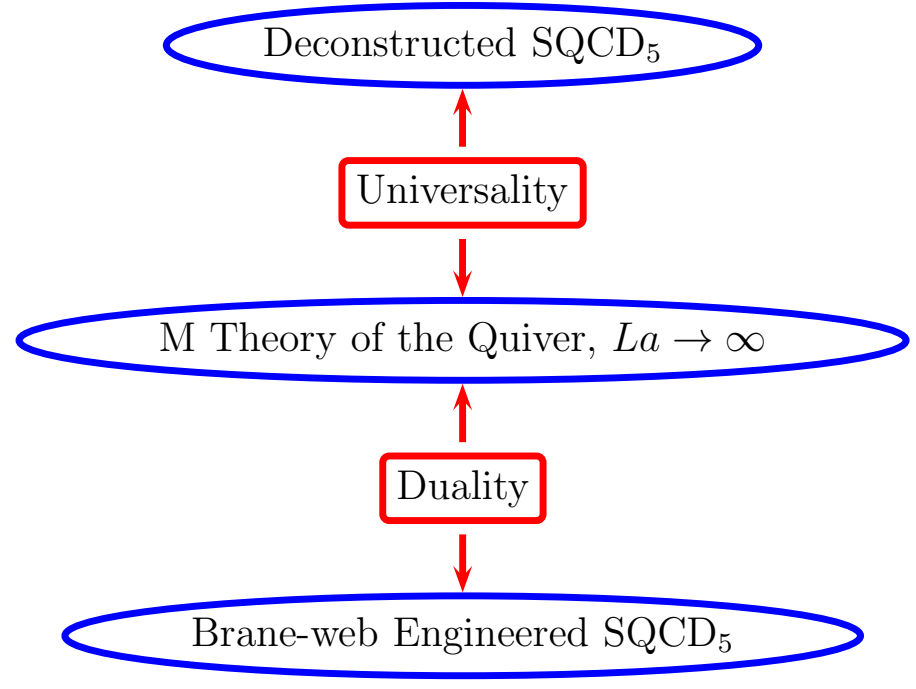

And since duality implies universality (but not the other way around), we find that the dimensional deconstruction and the brane-web engineering of the same $\mathrm{SQCD}_{5}$ are in the same universality class.

To prove the duality part of the diagram (7.3) we will show the following:

(1) For $L a \rightarrow \infty$, the spectral curve of the quiver becomes a union of linear segments $\alpha \xi-\beta \eta=$ const with integer $\alpha$ and $\beta$. The joints between the segments are infinitesimal but have $\delta$-like curvature, which allows different $(\alpha, \beta)$ for different segments.

(2) The coordinates $X^{9}=C \operatorname{Im} \xi$ and $X^{10}=C \operatorname{Im} \eta$ are periodic. Together they form a $T^{2}$ torus, and $\mathrm{M}$ theory on this torus is dual to the type IIB string theory on a circle $S^{1}$. Under this duality, the M5 brane's part spanning an $\alpha \xi-\beta \eta=$ const piece of the spectral curve (times the $\mathbb{R}^{3,1}$ Minkowski space) becomes the $(p=\alpha, q=\beta)$-brane spanning $\mathbb{R}^{3,1} \times S^{1} \times$ a real line segment $\alpha X^{5}-\beta X^{6}=$ const. And the M5 spanning the whole spectral curve is dual to a $(p, q)$ brane web made of such segments.

(3) This brane web turns out to be precisely the web implementing the $\mathrm{SQCD}_{5}$ with appropriate $n_{c}, n_{f}$, and $k_{\mathrm{cs}}$. (Except that one of the 5 dimensions is compactified 
on the $S^{1}$.) In particular, for positive enough coupling $h$, the web forms a ladder with $n_{c}$ parallel rungs; this implements the SQCD Coulomb phase of the 5D theory. For negative or low enough $h$, the web flips to a different topology, and this happens precisely when the deconstructed theory has a phase transition.

(4) Finally, comparing the semiclassical gauge boson masses in the M theory and in the deconstructed $\mathrm{SQCD}_{5}$ yields $C=\sqrt{L a / 2 \pi t_{3}}$ where $t_{3}$ is the M2 brane tension. This gives us the area of the $T^{2}$ torus in the $\mathrm{M}$ theory and hence the radius of the $S^{1}$ circle in the dual string theory [36]. That radius turns out to be $R=L a / 2 \pi$ - which is precisely the radius of the deconstructed dimension. Hence, in the decompactification limit of the deconstructed theory, the brane web also decompactifies to 5D.

So let us start with part (1) of our argument. Consider the spectral curve (4.21) as a quadratic equation for the $y(x)$ and let us take the $L a \rightarrow \infty$ limit for fixed $\xi$ and $\eta$. Similar to eqs. (4.39-44), the polynomials $p(x)$ and $b(x)$ become in this limit

$$
\begin{aligned}
p\left(x=e^{L a \xi}\right) \longrightarrow & \pm \exp \left[\operatorname{La} \times \sum_{i=1}^{n_{c}} \max (\xi, \varphi)\right] \\
& \text { as long as } \operatorname{Re} \xi \neq \text { any of the } \phi_{i}, \\
b\left(x=e^{L a \xi}\right) \longrightarrow & \pm \exp \left[\operatorname{La} \times\left(\Delta F \times \xi+\sum_{f=1}^{n_{f}} \max \left(\xi, m_{f}\right)\right)\right] \\
& \text { as long as } \operatorname{Re} \xi \neq \text { any of the } \operatorname{Re} m_{f} .
\end{aligned}
$$

(By abuse of notations, here max of two complex numbers denotes the number with the larger real part.) And as in eq. (4.32), for almost all $\xi$ either $p^{2}(x) \gg e^{-L a S} b(x)$ or else $p^{2}(x) \ll e^{-L a S} b(x)$. When $p^{2}(x) \gg e^{-L a S} b(x)$, eq. (4.21) for the $y(x)$ has two very different solutions, namely

$$
y_{1}(x)=p(x), \quad y_{2}(x)=\frac{e^{-L a S} b(x)}{p(x)}, \quad y_{1} \gg y_{2}
$$


in terms of $\eta(\xi)$, these solutions translate to

$$
\begin{aligned}
& \eta_{1}(\xi)=\sum_{i=1}^{n_{c}} \max (\xi, \varphi), \\
& \eta_{2}(\xi)=-S-\sum_{i=1}^{n_{c}} \max (\xi, \varphi)+\Delta F \times \xi+\sum_{f=1}^{n_{f}} \max \left(\xi, m_{f}\right) .
\end{aligned}
$$

Note that both $\eta_{1}$ and $\eta_{2}$ are piecewise-linear functions of $\xi$, and their derivatives are integervalued. (That is, for each piece $d \eta / d \xi$ is a constant integer, but its value jumps from piece to piece.) And when $p^{2}(x) \ll e^{-L a S} b(x)$, the two solutions of eq. (4.21) become

$$
\hat{y}_{1,2}(x)= \pm i e^{-L a S / 2} \sqrt{b(x)}
$$

or in terms of $\xi$ and $\eta$,

$$
\hat{\eta}_{1,2}(\xi)=-\frac{S}{2}+\frac{\Delta F}{2} \times \xi+\frac{1}{2} \sum_{f=1}^{n_{f}} \max \left(\xi, m_{f}\right) \pm \frac{\pi i}{2 L a}
$$

Again, $\hat{\eta}_{1,2}$ are piecewise-linear functions of $\xi$, and for each piece the derivative $d \hat{\eta}_{1,2} / d \xi$ is integer or half-integer. Consequently, the whole spectral curve consists of a bunch of linear pieces, and each piece is rational, $i$. e. satisfies $\alpha \xi-\beta \eta=$ const for some integers $\alpha$ and $\beta$.

Actually, eqs. (7.7-8) and (7.10) miss some of the pieces of the spectral curve, but the missing pieces are also linear and rational. These missing pieces are located at $\xi=\varphi_{i}$ or $\xi=m_{f}$ where the limits $(7.4-5)$ do not work. Instead, for $\xi$ in a $O(1 / L a)$ neighborhood of a modulus $\varphi_{i}$ the value of the $p(x)$ polynomial can be anywhere between zero and the right hand side of eq. (7.4); likewise, for $\xi$ in a $O(1 / L a)$ neighborhood of a mass $m_{f}$ the value of $b(x)$ can be anywhere between zero and the right hand side of eq. (7.5). Consequently, in the decompactification limit, a fixed value of $\xi$ which happens to coincide with a modulus $\varphi_{i}$ or a mass $m_{f}$ agrees with a wide range of values of $\eta \cdot{ }^{\langle 16\rangle}$ Thus, the spectral curve has several $\xi=$ const pieces — which are linear and rational with $(\alpha, \beta)=(1,0)$.

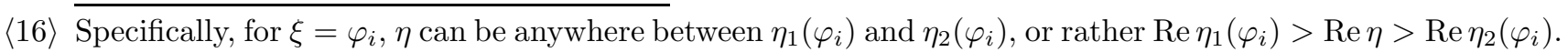
Likewise, for $\xi=m_{f}, \eta$ may vary from $\eta_{2}\left(m_{f}\right)$ or $\hat{\eta}_{2}\left(m_{f}\right)$ (whichever applies) all the way to $-\infty$. 
Altogether, the decompactification limit of the spectral curve (4.21) of the quiver is a union of segments of rational straight lines $\alpha \xi-\beta \eta=$ const. For large but finite $L a$, the joints between adjacent segments have small but finite sizes of the order $O(1 / L a)$. Such joints are strongly curved - curvature $=O(L a)$ - which allows for finite differences between directions $\alpha / \beta$ of segments they connect. For $L a \rightarrow \infty$, the joints' sizes become infinitesimal while the curvature becomes $\delta$-like. This completes part (1) of our proof.

Next, consider the spectral curve (4.21) as a Riemann surface. This surface is a double cover of the complex $x$ sphere, or in other words $x$ spans $\mathbb{C}^{*}$, twice. The map $x=\exp (L a \times \xi)$ takes out the $x=0$ and $x=\infty$ points, which turns the $x$ sphere into a complex cylinder: Re $\xi$ is single valued and spans the real line $\mathbb{R}$ while $\operatorname{Im} \xi$ is periodic modulo $(2 \pi / L a)$. Likewise, the map $y=\exp (L a \times \eta)$ turns the $y$ sphere into a cylinder: Re $\eta$ is single valued and spans $\mathbb{R}$ while $\operatorname{Im} \eta$ is periodic modulo $(2 \pi / L a)$. In terms of eqs. $(7.1)$, this means that $\left(X^{5}, X^{6}\right)$ span an infinite plain $\mathbb{R}^{2}$ while $\left(X^{9}, X^{10}\right)$ span a torus $T^{2}$ of area $A=(2 \pi C / L a)^{2}$.

M theory compactified on this torus is dual to the type IIB string theory ${ }^{\langle 17\rangle}$ compactified on a circle $S^{1}$ whose radius is inversely proportional to the torus's area,

$$
\frac{1}{R\left[S^{1}\right]}=t_{3} \times \operatorname{Area}\left[T^{2}\right] \equiv t_{3} \times(2 \pi C / L a)^{2}
$$

where $t_{3}$ is the membrane (M2) tension in M theory. Under this duality, an M5 brane which wraps one circle of the $T^{2}$ is dual to a $(p, q) 5$-brane which wraps the $S^{1}$ circle. The NeveuSchwarz-Ramond charges $(p, q)$ of this dual 5 -brane depend on a particular circle of the $T^{2}$ wrapped by the M5: for a circle $\alpha X^{9}-\beta X^{10}=$ const with integer $\alpha$ and $\beta$, the Ramond charge $p=\alpha$ and the Neveu-Schwarz charge $q=\beta$.

According to eqs. (7.1), a linear piece $\alpha \xi-\beta \eta=$ const of the spectral curve is the direct product of a real line segment $\alpha X^{5}-\beta X^{6}=$ const in the $X^{5,6}$ plane, times a circle

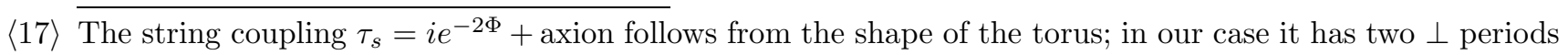
of equal length, hence $\tau_{s}=i$. We may choose a different $\tau_{s}$ by modifying eqs. (7.1) according to

$$
X^{5}+i X^{9}=(\xi+\text { axion } \times \eta) \times C e^{+\Phi}, \quad X^{6}+i X^{10}=\eta \times C e^{-\Phi} .
$$

However, in brane-web engineering the actual value of the $\tau_{s}$ is not important, and so we stick with eqs. (7.1) as they are and hence $\tau_{s}=i$. 
$\alpha X^{9}-\beta X^{10}=$ const in the $T^{2}$ torus. Hence, the M5 brane spanning this piece (times the $4 \mathrm{D}$ Minkowski space $\left.\mathbb{R}^{3,1}\right)$ is dual to the $(p=\alpha, q=\beta)$-brane which spans $\mathbb{R}^{3,1} \times S^{1} \times$ the real line segment $\alpha X^{5}-\beta X^{6}=$ const. And the M5 brane spanning the entire spectral curve of the quiver theory is dual to the $(p, q)$ brane web made of such segments. This completes part (2) of our proof.

Now consider the web's geometry for different phases of the deconstructed 5D theory. Let us start with the ordinary $S Q C D_{5}$ phase where branching points of the $4 \mathrm{D}$ spectral curve over the $x$ plane form $n$ very close pairs (4.35-36). This pattern requires $\operatorname{Re} S=h+\frac{1}{2} \sum m_{f}$ large enough to assure that $p^{2}(x) \gg e^{-L a S} b(x)$ for all $x=e^{L a \xi}$ (except when $\xi$ equals one of the $\varphi_{i}$ ). Consequently, the spectral curve follows eqs. (7.6) rather than (7.9) for all $x$, or in terms of $\xi$ and $\eta$, it comprises complex lines $\eta_{1}(\xi)$ and $\eta_{2}(\xi)$ according to eqs. (7.7-8) for all $\xi$. And there are also $\xi=$ const complex lines for $\xi=\varphi_{i}$ and $\xi=m_{f}$. The brane web dual to this curve spans the real parts of all these complex lines, thus:

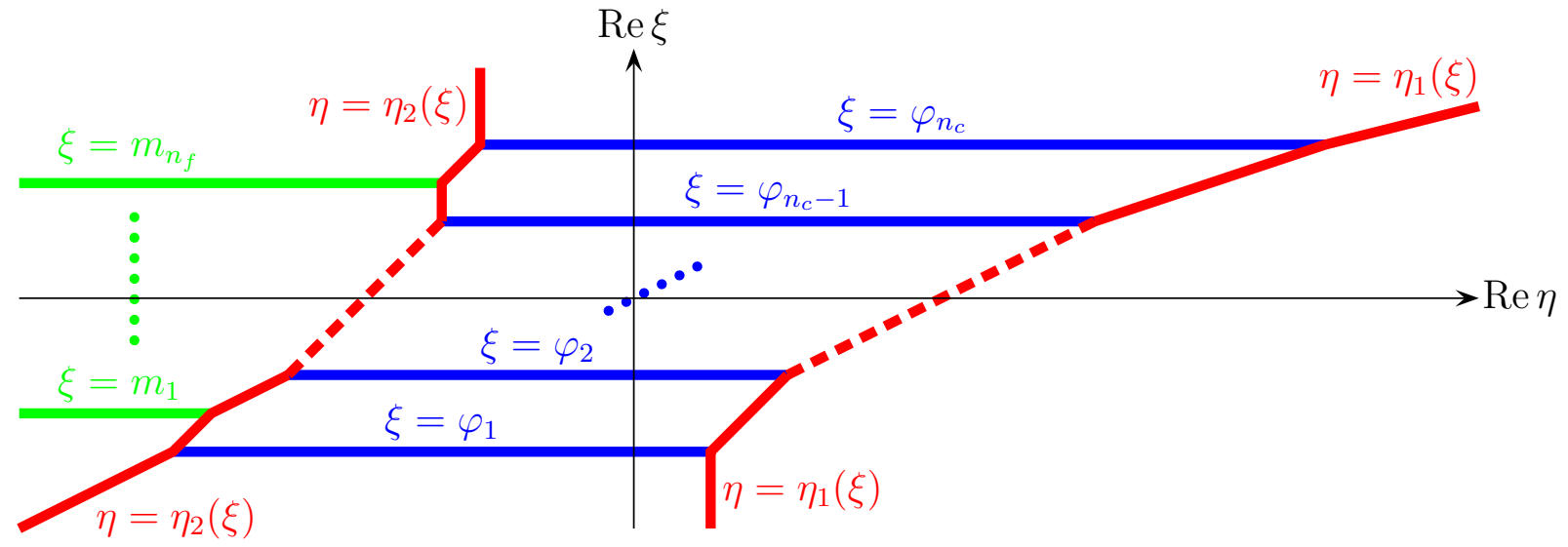

As promised, this web looks like a ladder with $n_{c}$ horizontal rungs (blue lines for $\xi=\varphi_{i}$ ) between two multiply-bent sides (red lines for $\eta=\eta_{1}(\xi)$ and $\eta=\eta_{2}(\xi)$ ), but there also are $n_{f}$ semi-infinite horizontal branes attached to the left side of the ladder (green lines for $\xi=m_{f}$ ). In 5D (after eventual decompactification of the $S^{1}$ circle), this web obviously gives rise to the Coulomb phase of an $\mathrm{SQCD}_{5}$ with $n_{c}$ colors and $n_{f}$ flavors. ${ }^{\langle 18\rangle}$ The Chern-Simons level

$\langle 18\rangle$ Indeed, strings between the 'blue' branes produce vector multiplets in the adjoint of $U\left(n_{c}\right)$ (spontaneously broken to $U(1)^{n_{c}}$ by distances $\phi_{i}-\phi_{j}$ between the branes), with the overall $U(1)$ frozen due to interactions with the 'red' branes (the sides of the ladder). Likewise, strings between the 'green' branes try to produce 
of this $\mathrm{SQCD}_{5}$ is less obvious, but it is related to the asymmetry between the top and the bottom ends of the ladder: At the top of the ladder $(\operatorname{Re} \xi \rightarrow+\infty)$, its two sides separate from each other at the rate

$$
\text { rate }_{\text {top }}=\left[\frac{d \eta_{1}}{d \xi}-\frac{d \eta_{2}}{d \xi}\right]_{\operatorname{Re} \xi \rightarrow+\infty}=\left(n_{c}\right)-\left(n_{f}+\Delta F-n_{c}\right)
$$

while at the bottom of the ladder they separate at a different rate

$$
\text { rate }_{\text {bottom }}=\left[\frac{d \eta_{2}}{d \xi}-\frac{d \eta_{1}}{d \xi}\right]_{\operatorname{Re} \xi \rightarrow-\infty}=(\Delta F)-(0)
$$

The Chern-Simons level is one half of the difference between these rates,

$$
k_{\mathrm{cs}}=\frac{1}{2}\left[\text { rate }_{\mathrm{top}}-\text { rate }_{\text {bottom }}\right]=n_{c}-\frac{1}{2} n_{f}-\Delta F
$$

note that it comes out exactly as in dimensional deconstruction, cf. eq. (3.27). Thus, the web (7.13) indeed gives rise to the same $\mathrm{SQCD}_{5}$ as the deconstructed theory we have started from, at least in the ordinary $S Q C D_{5}$ Coulomb phase.

In terms of the web (7.13), lowering the $h$ parameter of the $5 \mathrm{D}$ theory makes the left side of the ladder move right, $c f$. eq. (7.8). Eventually, for some critical $h=h_{c}$, the left side collides with the right side, and then the web switches to a different configuration. The details of this transition depend on whether the two sides of the ladder collide at a single point or over some length of parallel segments. For a point collision, the web has a flop

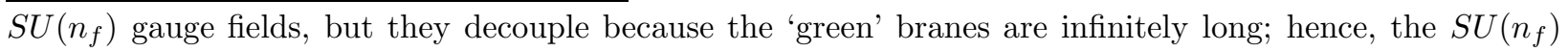
symmetry is flavor rather than gauge. Finally, $n_{c} \times n_{f}$ quark hypermultiplets arise from strings connecting the blue and the green branes to each other. 
transition

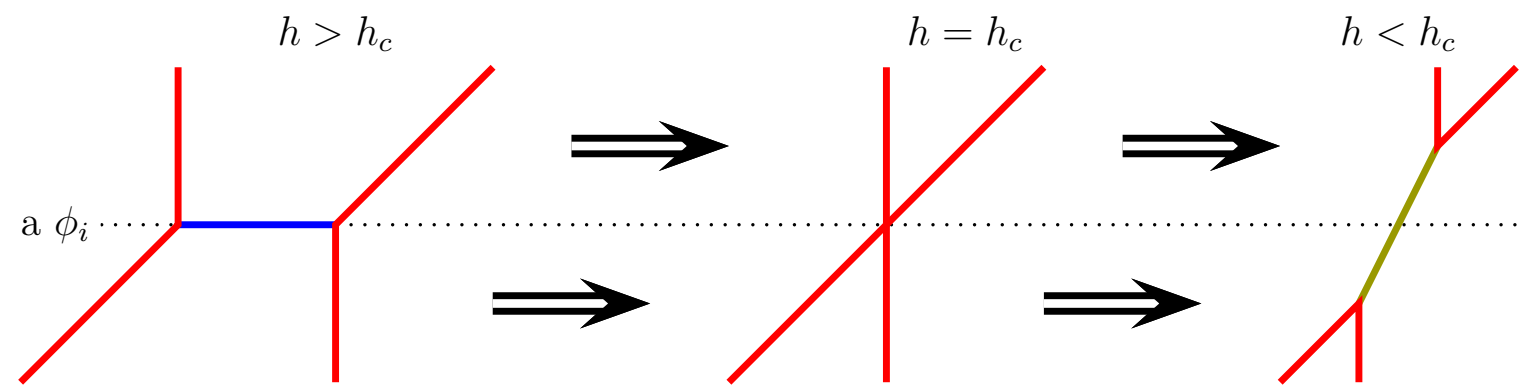

(Note that this picture shows only a part of the web.) For a parallel-line collision, we have a more complicated picture:

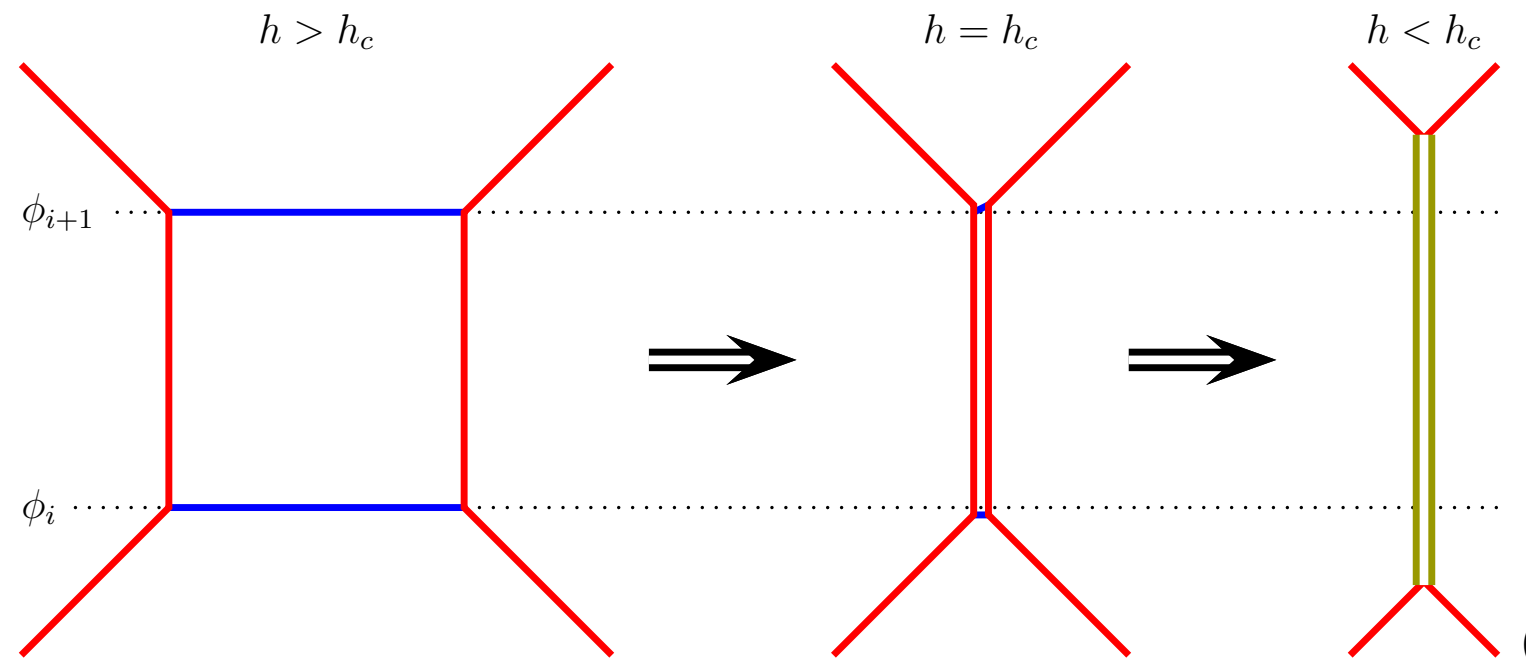

(again, only a part of the web is shown). This time, the web does not change topology for $h<h_{c}$; instead, the two coincident segments (colored brown in the above picture) are no longer bounded by $\phi_{i}<\operatorname{Re} \xi<\phi_{i+1}$ but become longer and longer with decreasing $h$.

From the spectral curve's point of view, the brown segments of the webs (7.17) and (7.18) for $h<h_{c}$ correspond to $\eta(\xi)$ following eqs. (7.10) instead of eqs. (7.7-8). Pictorially, we 
have

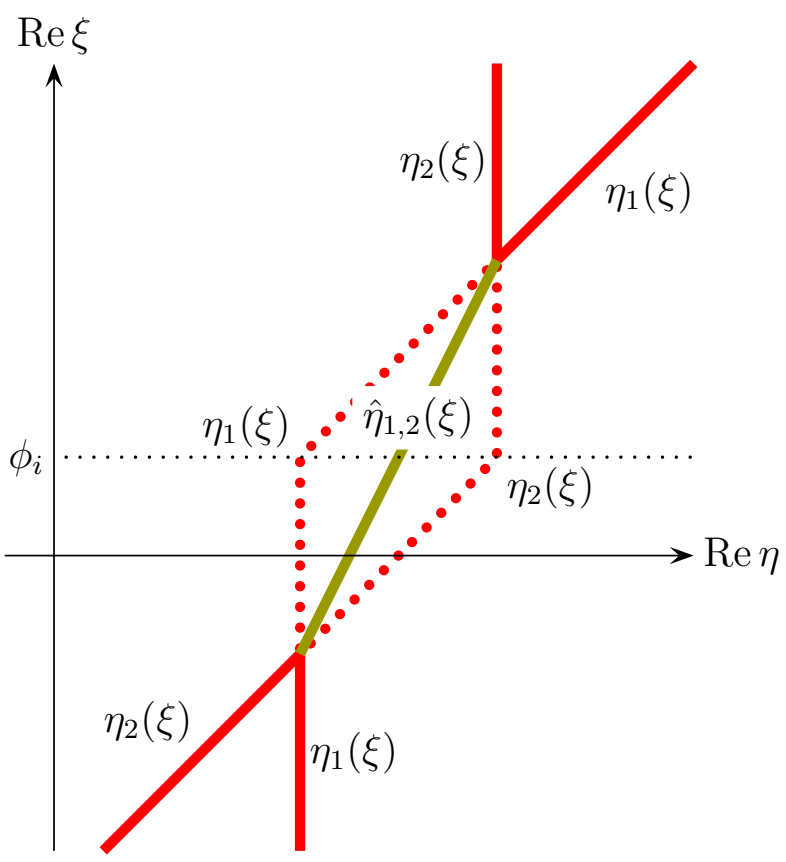

for the right web (7.17), and

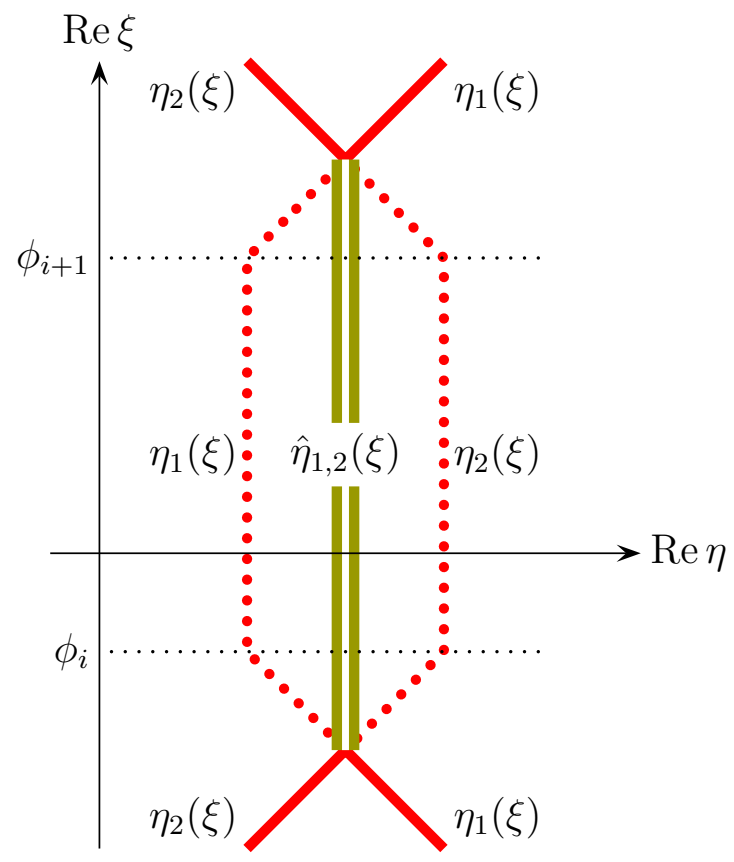

for the right web (7.18); in both figures, the dotted red lines plot eqs. (7.7-8) in the range of $\operatorname{Re} \xi$ where those equations do not apply. Indeed, the $L a \rightarrow \infty$ limit of the spectral curve follows eqs. (7.7-8) only when $p^{2}(x) \gg e^{-L a S} b(x)$; in terms of eqs. (7.7-8) themselves, 
this corresponds to $\operatorname{Re} \eta_{1}(\xi)>\operatorname{Re} \eta_{2}(\xi)$. But for $h<h_{c}$, there is a range of $\operatorname{Re} \xi$ for which $\operatorname{Re} \eta_{1}(\xi)<\operatorname{Re} \eta_{2}(\xi)$ - cf. the dotted red lines in figures (7.19-20) - and in this range $p^{2}(x) \ll e^{-L a S} b(x)$ and the spectral curve follows eqs. (7.10) instead of eqs. (7.7-8). Note that the switchover is continuous because

$$
\hat{\eta}_{1,2}(\xi)=\frac{\eta_{1}(\xi)+\eta_{2}(\xi)}{2} \pm \frac{\pi i}{2 L a}
$$

The $(p, q)$ 5-branes dual to the $\hat{\eta}_{1,2}(\xi)$ segments of the curve can be single as in figure (7.19) or double (two coincident branes) as in figure (7.20). The factor deciding between single or double branes is the derivative $d \hat{\eta}_{1,2} / d \xi$, which is quantized in units of $\frac{1}{2}$ : if it is integer the brane is double, and if it is half-integer the brane is single. To see this, consider the imaginary parts of the $\hat{\eta}_{1}(\xi)$ and $\hat{\eta}_{2}(\xi)$ segments of the spectral curve. For an integer $d \hat{\eta}_{1,2} / d \xi$, the line $\left(\operatorname{Im} \xi, \operatorname{Im} \hat{\eta}_{1}(\xi)\right)$ on the torus $T^{2}$ is a complete circle, and the line $\left(\operatorname{Im} \xi, \operatorname{Im} \hat{\eta}_{2}(\xi)\right)$ is a separate complete circle; the two circles are parallel but separated from each other by half-a-period in the $\operatorname{Im} \eta$ direction, $c f$. eq. (7.21). Together, the M5 branes wrapping these two circles are dual to two 5 -branes with similar $(p, q)$ charges, and the positions of these two branes coincide because $\operatorname{Re} \hat{\eta}_{1}(\xi)=\operatorname{Re} \hat{\eta}_{2}(\xi)$. On the other hand, when $d \hat{\eta}_{1,2} / d \xi$ is a half-integer, the lines $\left(\operatorname{Im} \xi, \operatorname{Im} \hat{\eta}_{1}(\xi)\right)$ and $\left(\operatorname{Im} \xi, \operatorname{Im} \hat{\eta}_{2}(\xi)\right)$ on the torus are two halves of the same circle. Consequently, the M5 brane wrapping this circle is dual to a single $(p, q)$ 5-brane.

In any case, the very existence of branes dual to $\hat{\eta}_{1,2}(\xi)$ instead of un-hatted $\eta_{1,2}(\xi)$ indicates that there is a range of $\xi$ for which $p^{2}(x) \ll e^{-L a S} b(x)$. By reasons of concavity this range must include at least one modulus $\varphi_{i}$, and consequently some of the branching points of the spectral curve over the $x$ plane do not form close pairs (4.35-36). Therefore, some of the deconstructed 5D gauge couplings deviate form eqs. (4.46-47), which means that the deconstructed theory is no longer in the ordinary $S Q C D_{5}$ Coulomb phase. Instead, we have a Coulomb phase of an exotic $5 \mathrm{D}$ theory such as the $E_{0}$ Coulomb phase of the $\tilde{D}_{0}$ model of $\S 6.1$, or perhaps an unphysical phase such as in the $D_{0}$ model of $\S 6.2$.

In general, distinct Coulomb phases of the deconstructed theory correspond to distinct patterns of the spectral curve's branching points $x_{1}, \ldots, x_{2 n_{c}}$. The duality translates these 
branching points into specific features of the brane web: a rung of the ladder

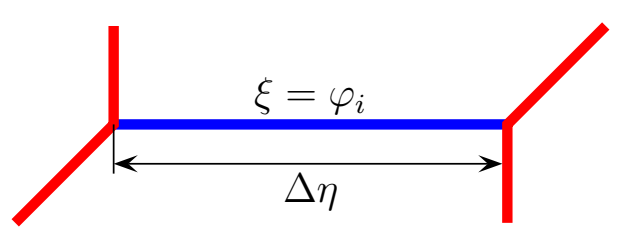

corresponds to a close pair

$$
x_{2 i-1}, x_{2 i}=e^{L a \varphi_{i}} \times\left(1 \pm e^{-L a \Delta \eta}\right) ;
$$

a joint where two sides of the ladder merge into a single brane dual to $\hat{\eta}_{1,2}(\xi)$
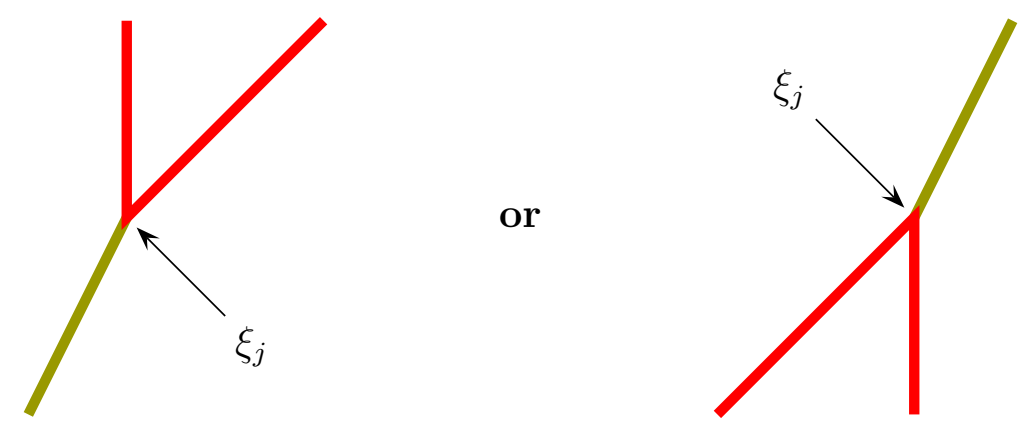

corresponds to an un-paired branching point at $x=e^{L a \xi_{j}}$; a joint involving a double brane
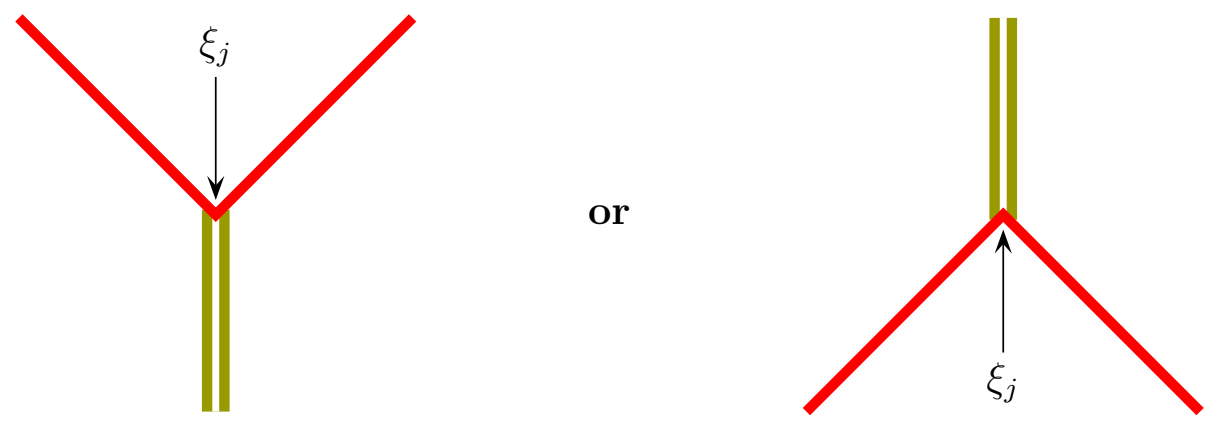

corresponds to two branching points at $x= \pm e^{L a \xi_{j}}$; and finally, a joint hiding collapsed 
cycles of the web such as

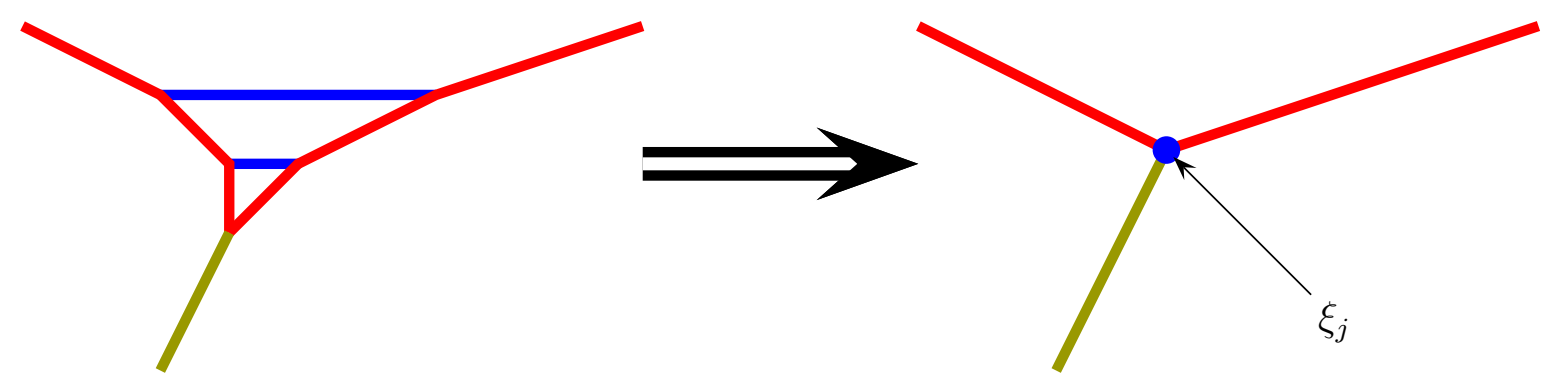

corresponds to $K$ branching points

$$
x_{\nu}=e^{L a \xi_{j}} \times e^{2 \pi i \nu / K}, \quad \nu=1,2, \ldots, K
$$

where

$$
K=2 \times \#\{\text { hidden cycles }\}+ \begin{cases}1 & \text { for a single } \hat{\eta}_{1,2}(\xi) \text { brane } \\ 2 & \text { for a double } \hat{\eta}_{1,2}(\xi) \text { brane }\end{cases}
$$

Although only the real parts of $\Delta \eta$ or $\xi_{j}$ are visible in the brane web, this gives enough information to identify the pattern of branching points - and hence the phase of the deconstructed theory - and even to calculate the matrix of 5D abelian gauge couplings. Thus, the phases of the deconstructed theory are in perfect correspondence with the phases of the brane web, and the transition between those phase happen at exactly the same $h_{c}$.

This completes part (3) of our proof.

Finally, let us calculate the $C$ parameter in eqs. (7.1). This parameter does not depend on the moduli, quark masses, or gauge coupling of the $5 \mathrm{D}$ theory, so let us make all the $\phi_{i}$ distinct to break $S U\left(n_{c}\right) \rightarrow U(1)^{n_{c}-1}$ and take the $h \rightarrow+\infty$ limit. This makes the quiver theory weakly coupled and allows semiclassical analysis. Consequently, the universality between the $4 \mathrm{D}$ gauge theory and the M theory on the M5 spanning the quiver's spectral curve should extend beyond the purely holomorphic data to other low-energy properties such as masses of light particles. In particular, $\mathrm{M}$ theory should reproduce the semiclassical mass $M=\left|\phi_{i}-\phi_{j}\right|$ of a non-abelian gauge boson $A_{i j}^{\mu}$.

In string theory on the brane web (7.13), this vector field arises from the string connecting 
appropriate rungs of the ladder,

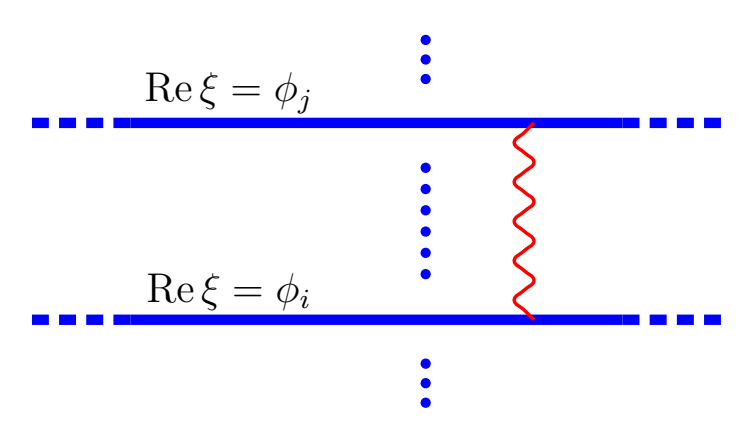

In $\mathrm{M}$ theory, this string is dual to $\mathrm{M} 2$ brane forming a cylinder: its long dimension is $X^{5}=C \times \operatorname{Re} \xi$ and the circular dimension is $X^{10}=C \times \operatorname{Im} \eta$. The mass of this tube is

$$
M=t_{3} \times \text { Area }=t_{3} \times C\left|\phi_{i}-\phi_{j}\right| \times \frac{2 \pi C}{L a}
$$

where $t_{3}$ is the membrane tension; equating this mass to the field-theoretical mass $M=$ $\left|\phi_{i}-\phi_{j}\right|$ of the vector field we arrive at

$$
C=\sqrt{\frac{L a}{2 \pi t_{3}}} .
$$

Given this value of $C$, eq. (7.12) tells us that the string theory dual to $\mathrm{M}$ theory is compactified on the circle $S^{1}$ of radius

$$
R=\frac{1}{t_{3}} \times\left(\frac{L a}{2 \pi C}\right)^{2}=\frac{L a}{2 \pi}
$$

- which is precisely the radius of the deconstructed dimension for finite $L$. And this completes the last part (4) of our proof.

To summarize, we have established that dimensional deconstruction and brane-web engineering of the same $\mathrm{SQCD}_{5}$ are always in the same universality class. And of course, other string/M implementations of $\mathrm{SQCD}_{5}$ are also in the same universality class because they are dual to the brane-web engineering. Which means that the phase diagram and other zeroenergy features of a 5D gauge theory are inherent properties of the theory itself, regardless of its UV completion. 
And this completes our analysis of quantum deconstruction. We showed how to use dimensional deconstruction as a UV completion of a 5D SUSY gauge theory such as $\mathrm{SQCD}_{5}$. We showed how to extract 5D quantum effects such as loop corrections to the prepotential from the 4D loop and instantonic effects - which can be calculated exactly thanks to the unbroken $\mathcal{N}=1$ SUSY in $4 \mathrm{D}$. We showed how to deconstruct the 5D phase diagrams, including the non-classical $h<0$ phases. And at the end of the story, the dimensional deconstruction turned out to be in the same universality class as the string-theoretical UV completions of the same 5D theory.

ACKNOWLEDGEMENTS: The authors thank Amer Iqbal and Jacob Sonnenschein for many interesting discussions. V. K. thanks the HEP theory group at Tel Aviv University for hospitality during his many visits there.

The research of V. K. is supported in part by the US National Science Foundation under grant PHY-0455649. The research of E. dN. is supported by the US Department of Energy under grant DE-FG02-06ER41418. 


\section{References}

[1] Nathan Seiberg, "Five Dimensional SUSY Field Theories, Non-Trivial Fixed Points, and String Dynamics", Phys. Lett. B 388 (1996) 753 arXiv:hep-th/9608111.

[2] David R. Morrison and Nathan Seiberg, "Extremal Transitions and FiveDimensional Supersymmetric Field Theories", Nucl. Phys. B 483 (1997) 229, arXiv:hep-th/9609070.

[3] A. C. Cadavid, A. Ceresole, R. D'Auria, and S. Ferrara, "11-Dimensional Supergravity Compactified on Calabi-Yau Threefolds", Phys. Lett. B357 (1995) 76, arXiv:hep-th/9506144

[4] Sergio Ferrara, Ruben Minasian, and Augusto Sagnotti, "Low-Energy Analysis of $M$ and F Theories on Calabi-Yau Threefolds", Nucl. Phys. B 474 (1996) 323, arXiv:hep-th/9604097

[5] K. Intriligator, D. R. Morrison and N. Seiberg, "Five-Dimensional Supersymmetric Gauge Theories and Degeneration of Calabi-Yau Spaces”, Nucl. Phys. B 497 (1997) 56, arXiv:hep-th/9702198.

[6] Sergio Ferrara, Ramzi R. Khuri, and Ruben Minasian, "M theory on a Calabi-Yau manifold", Phys. Lett. B 375 (1996) 81, arXiv:hep-th/9602102.

[7] Ori J. Ganor, David R. Morrison and Nathan Seiberg, "Branes, Calabi-Yau Spaces, and Toroidal Compactifications of the $\mathcal{N}=1$ Six-Dimensional E $E_{8}$ Theory", Nucl. Phys. B 487 (1997) 93, arXiv:hep-th/9610251.

[8] Ofer Aharony and Amihay Hanany, "Branes, Superpotentials, and Superconformal Fixed Points", Nucl. Phys. B 504 (1997) 239, arXiv:hep-th/9704170.

[9] Ofer Aharony, Amihay Hanany, and Barak Kol, "Webs of ( $p, q)$ 5-Branes, Five Dimensional Field Theories, and Grid Diagrams", JHEP 9801 (1998) 002, arXiv:hep-th/9710116. 
[10] Oliver DeWolfe, Amihay Hanany, Amer Iqbal, Emanuel Katz, "Five-branes, Seven-branes, and Five-dimensional $E_{n}$ Field Theories", JHEP 9903 (1999) 006, arXiv:hep-th/9902179.

[11] N. C. Leung and C. Vafa, "Branes and Toric Geometry", Adv. Theor. Math. Phys. 2 (1998) 91, arXiv:hep-th/9711013.

[12] Barak Kol and J. Rahmfeld, "BPS Spectrum of 5 Dimensional Field Theories, $(p, q)$ Webs, and Curve Counting”, JHEP 9808 (1998) 006, arXiv:hep-th/9801067.

[13] M. R. Douglas, S. Katz and C. Vafa, "Small Instantons, del Pezzo Surfaces, and Type I' Theory", Nucl. Phys. B 497 (1997) 155, arXiv:hep-th/9609071.

[14] Edward Witten, "Phase Transitions in M-Theory and F-Theory", Nucl. Phys. B 471 (1996) 195, arXiv:hep-th/9603150.

[15] Nima Arkani-Hamed, Andrew G. Cohen, and Howard Georgi, "(De)Constructing Dimensions", Phys. Rev. Lett. 86 (2001) 4757, arXiv:hep-th/0104005.

[16] Christopher T. Hill, Stefan Pokorski, and Jing Wang, "Gauge Invariant Effective Lagrangian for Kaluza-Klein Modes", Phys. Rev. D 64 (2001), 105005, arXiv:hep-th/0104035.

[17] Nima Arkani-Hamed, Andrew G. Cohen, and Howard Georgi, "Electroweak Symmetry Breaking from Dimensional Deconstruction", Phys. Lett. B 513 (2001) 232, arXiv:hep-ph/0105239

[18] Hsin-Chia Cheng, Christopher T. Hill, and Jing Wang, "Dynamical Electroweak Breaking and Latticized Extra Dimensions", Phys. Rev. D 64 (2001) 095003, arXiv:hep-ph/0105323

[19] C. Csaki, J. Erlich, C. Grojean, and G. Kribs, "4D Constructions of Supersymmetric Extra Dimensions and Gaugino Mediation," Phys. Rev. D 65 (2002) 015003, arXiv: hep-ph/0106044. 
[20] Amer Iqbal and Vadim S. Kaplunovsky, "Quantum Deconstruction of a 5D SYM and its Moduli Space," JHEP 0405 (2004) 013, arXiv:hep-th/0212098.

$\S 3.2$ of this paper — deconstructing Chern-Simons — was done in collaboration with Edoardo Di Napoli.

[21] N. Seiberg, "Naturalness versus Supersymmetric Non-Renormalization Theorems", Phys. Lett. B 318 (1993) 469, arXiv:hep-ph/9309335

[22] P. C. Argyres, M. R. Plesser, and N. Seiberg, "The Moduli Space of Vacua of $\mathcal{N}=2$ SUSY QCD and Duality in $\mathcal{N}=1$ SUSY QCD”, Nucl. Phys. B 471 (1996) 159, arXiv:hep-th/9603042.

[23] C. Csaki, J. Erlich, V. V. Khoze, E. Poppitz, Y. Shadmi, and Y. Shirman, "Exact Results in 5D from Instantons and Deconstruction," Phys. Rev. D 65 (2002) 085033 , arXiv:hep-th/0110188.

[24] Csaba Csaki, Joshua Erlich, Daniel Freedman, and Witold Skiba, "N $=1$ Supersymmetric Product Group Theories in the Coulomb Phase", Phys. Rev. D 56 (1997) 5209, arXiv:hep-th/9704067.

[25] Edoardo Di Napoli, Vadim S. Kaplunovsky, and Jacob Sonnenschein, "Chiral Rings of Deconstructive $\left[S U\left(n_{c}\right)\right]^{N}$ Quivers," JHEP 0406 (2004) 060, arXiv:hep-th/0406122.

[26] Edward Witten, "Solutions Of Four-Dimensional Field Theories Via M Theory", Nucl. Phys. B 500 (1997) 3, arXiv:hep-th/9703166.

[27] Amihay Hanany, Mathew J. Strassler, and Alberto Zaffaroni, "Confinement and Strings in MQCD”, Nucl. Phys. B 513 (1998) 87, arXiv:hep-th/9707244

[28] A. Brandhuber, N. Itzhaki, V. Kaplunovsky,J. Sonnenschein, and S. Yankielowicz, "Comments on the $M$ Theory Approach to $\mathcal{N}=1$ SQCD and Brane Dynamics", Phys. Lett. B 41 (1997) 27, arXiv:hep-th/9706127

[29] Michael R. Douglas and Gregory Moore, D-branes, Quivers, and ALE Instantons", arXiv:hep-th/9603167 
[30] Nima Arkani-Hamed, Andrew G. Cohen, David B. Kaplan, Andreas Karch, and Luboš Motl, "Deconstructing (2,0) and Little String Theories", JHEP 0301 (2003) 083, arXiv:hep-th/0110146.

[31] Eric G. Gimon and Joseph Polchinski, "Consistency Conditions for Orientifolds and D-Manifolds," Phys. Rev. D 54 (1996) 1667 arXiv:hep-th/9601038.

[32] L. E. Ibanez, R. Rabadan, and A. M. Uranga, "Anomalous U(1)'s in Type I and Type IIB $D=4, \mathcal{N}=1$ String Vacua," Nucl. Phys. B542 (1999) 112, arXiv:hep-th/9808139.

[33] Witold Skiba and David Smith, "Localized Fermions and Anomaly Inflow via Deconstruction," Phys. Lett. D65 (2002) 095, arXiv:hep-ph/0201056.

[34] Jerome P. Gauntlett, Dario Martelli, James Sparks, and Daniel Waldram, "Supersymmetric $\mathrm{AdS}_{5}$ Solutions of M-Theory", Class. Quant. Grav. 21 (2004) 4335, arXiv:hep-th/0402153; "Sasaki-Einstein Metrics on $S^{2} \times S^{3}$ ", Adv. Theor. Math. Phys. 8 (2004) 711, arXiv:hep-th/0403002.

[35] Amihay Hanany, Pavlos Kazakopoulos, Brian Wecht, "A New Infinite Class of Quiver Gauge Theories", JHEP 0508 (2005) 054, arXiv:hep-th/0503177.

[36] Edward Witten, "String Theory Dynamics in Various Dimensions", Nucl. Phys. B 443 (1995) 85, arXiv:hep-th/9503124 